\title{
Quadratic Representations of the Canonical Commutation Relations
}

\author{
By \\ Martin ProksCH*, George ReEnTS** and Stephen J. SummerS***
}

\begin{abstract}
This paper studies a class of representations (called quadratic) of the canonical commutation relations over symplectic spaces of arbitrary dimension, which naturally generalizes coherent and symplectic (i.e. quasifree) representations and which has previously been heuristically employed in the special case of finite degrees of freedom in the physics literature. An explicit characterization of canonical quadratic transformations in terms of a 'standard form' is given, and it is shown that they can be exponentiated to give representations of the Weyl algebra. Necessary and sufficient conditions are presented for the unitary equivalence of these representations with the Fock representation. Possible applications to quantum optics and quantum field theory are briefly indicated.
\end{abstract}

\section{Introduction}

Since the canonical commutation relations (henceforth, the CCR) were introduced by Dirac in 1927 as generalizations of Heisenberg's commutation relations in order to discuss radiation theory [6], they have received a great deal of attention. Rigorous mathematical analysis of the CCR for the purposes of quantum field theory began in the 1950's, when it was also realized that in the case of infinitely many degrees of freedom, which is of relevance in quantum statistical mechanics and quantum field theory, there are (uncountably) infinitely many inequivalent irreducible representations of the CCR [8], and that the choice of the proper representation is crucial in any physical application. Various classes of representations of the CCR have been introduced and studied, and in this paper we wish to examine mathematically another class of representations. We

Communicated by H. Araki, March 7, 1994.

1991 Mathematics Subject Classification (s): 81 S05

* Physikalisches Institut, Universität Würzburg, D-97074, Würzburg, Germany

** Physikalisches Institut, Universität Würzburg, D-97074, Würzburg, Germany

*** Department of Mathematics, University of Florida, Gainesville, FL 32611, USA 
shall introduce this additional class in a setting that makes clear that it is a natural generalization of two classes that have been intensively studied earlier the coherent representations and the symplectic representations (also called quasifree representations). It should be mentioned that special cases of this class of representations have already been introduced and heuristically studied in the physics literature for a single degree of freedom [3] [16] and for a finite number of degrees of freedom [5]. We give here the first mathematically rigorous treatment of the entire class of quadratic representations, and we do so without restriction on the number of degrees of freedom.

We first give a brief introduction to these classes of representations. Let $H$ be a real test function space with a symplectic form $\sigma$ defined on it, and let $\{\Phi(f) \mid f \in H\}$ be a family of densely defined linear operators acting on the complex Hilbert space $\mathscr{K}$ and satisfying

$$
[\Phi(f), \Phi(g)] \subset i \sigma(f, g) \mathbf{1}, \quad f, g \in H
$$

In other words, $\Phi(f)$ is a representation of the CCR over $(H, \sigma)$. The best known such representation is that of Fock (cf. [4] for the first mathematically rigorous discussion of the Fock representation). Representations of the CCR are the basic building blocks in the theory of bosonic systems. The number of physical degrees of freedom of the system determines the dimension of $H-$ in particular, dealing with infinitely many degrees of freedom necessitates handling infinite dimensional spaces $H$, whence the mathematical problems alluded to above arise. Different, i.e. unitarily inequivalent, representations of the CCR over $(H, \sigma)$ manifest different physical properties in general. Roughly speaking, the choice of the symplectic space $(H, \sigma)$ corresponds to a choice of kinematics in the model, whereas the choice of representation corresponds to a choice of dynamics. (For an elaboration of this point, see the texts [7] [25] [9]). A standard technique for constructing such representations is to start with a Fock representation and then perform a canonical transformation, i.e. transformation $\Phi(f) \mapsto \Phi^{\prime}(f), f \in H$, such that equation (1.1) holds with $\Phi$ replaced by $\Phi^{\prime}$. If the new representation $\Phi^{\prime}(f)$ is not unitarily equivalent to $\Phi(f)$, then one has the chance of modelling different physics. In this paper, a new class of representations of the CCR, which are not unitarily equivalent to the Fock representation and which therefore open up the opportunity to model new physics, is displayed.

The simplest class of canonical transformations is that of the coherent transformations

$$
\Phi(f) \mapsto \Phi^{\prime}(f) \equiv \Phi(f)+\lambda(f) \mathbb{1}
$$

where $\lambda$ is a real-valued functional on $H$, which are intimately related with the 
coherent states used in quantum optics and elsewhere [12]. Another well-studied class of canonical transformations is that of the symplectic (or quasifree, or Bogoliubov) transformations

$$
\Phi(f) \mapsto \Phi^{\prime}(f) \equiv \Phi(T f)=\Phi(f)+\Phi((T-1) f),
$$

where the operator $T: H \mapsto H$ is symplectic, i.e. satisfies $\sigma(T f, T g)=\sigma(f, g)$. Together, the coherent and symplectic transformations constitute the group of inhomogeneous linear canonical transformations, have been intensively studied (see, for example, [1]), and have found application in many branches of theoretical physics. It is known that the coherent representations are unitarily equivalent to the Fock representation if and only if the linear functional $\lambda$ is bounded [1], and the symplectic transformations are unitarily equivalent to the original Fock representation if and only if the operator $1-|T|=1-\left(T^{*} T\right)^{1 / 2}$ is a Hilbert-Schmidt operator [26].

To avoid technical definitions (which will be given later in the body of this paper) in this introduction, we shall illustrate some of the basic points to be made with a class of easily describable examples. Let $\left\{q_{k}, p_{k}\right\}_{k=1}^{\infty}$ be a system of densely defined operators acting on the complex Hilbert space $\mathscr{K}$ and satisfying

$$
\left[q_{j}, p_{k}\right]=i \delta_{j k} \cdot \mathbf{1}
$$

and for the standard annihilation and creation operators $a_{k} \equiv \frac{1}{\sqrt{2}}\left(q_{k}+i p_{k}\right)$ and $a_{k}^{\dagger} \equiv \frac{1}{\sqrt{2}}\left(q_{k}-i p_{k}\right)$ let there be a vector $\Omega \in \mathscr{K}$ such that $a_{k} \Omega=0$ for all $k \in \mathbf{N}$. In other words, we consider the Fock representation of a bosonic system with infinitely many degrees of freedom. In this setting, the coherent, resp. symplectic, canonical transformations can be written as

$$
\begin{array}{ll}
\text { (A) } q_{k} \mapsto q_{k}, & p_{k} \mapsto p_{k}+\lambda_{k} \cdot 1, \\
\text { (B) } \quad q_{k} \mapsto q_{k}, & p_{k} \mapsto p_{k}+\sum_{l} \lambda_{k l} q_{l},
\end{array}
$$

where $\lambda_{k}, \lambda_{k l}$ are real and $\lambda_{k l}=\lambda_{l k}$ for each $k, l$. Both transformations have the general structure $q_{k} \mapsto q_{k}, p_{k} \mapsto p_{k}+F_{k}\left(q_{1}, q_{2}, \ldots\right)$, where the $F_{k}\left(q_{1}, q_{2}, \ldots\right)$ are real functions of the operators $q_{1}, q_{2}, \ldots$. Note that the transformations above are generated by the choice $F_{k} \equiv \frac{\partial F}{\partial x_{k}}$, with

$$
\begin{aligned}
& \text { (A) } F\left(x_{1}, x_{2}, \ldots\right)=\sum_{k} \lambda_{k} x_{k} \\
& \text { (B) } F\left(x_{1}, x_{2, \ldots}\right)=\frac{1}{2} \sum_{k, l} \lambda_{k l}: x_{k} x_{l}: \equiv \frac{1}{2} \sum_{k, l} \lambda_{k l}\left(x_{k} x_{l}-\frac{1}{2} \delta_{k l}\right) .
\end{aligned}
$$


It is a natural next step to choose $F=\frac{1}{3} \sum_{k, l, m} \lambda_{k l m}: x_{k} x_{l} x_{m}:$, which would give rise to the transformation [22]

$$
q_{k} \mapsto q_{k}, \quad p_{k} \mapsto p_{k}+\sum_{l, m} \lambda_{k l m}: q_{l} q_{m}:
$$

(In order for this transformation to be canonical, the coefficients $\lambda_{k l m} \in \mathbb{R}$ must be totally symmetric in the indices.) We find it natural to call these transformations (and the resulting representations) higher-order ${ }^{1}$ transformations (representations) of the CCR.

After defining general quadratic transformations below, those which are canonical will be characterized. It will be shown that for any canonical quadratic transformation, there exists a choice of basis in $(H, \sigma)$ in terms of which the transformation can be brought into the form $(1.2)^{2}$ with $\sum_{l, m} \lambda_{k l m}^{2}<\infty$. Moreover, it will be proven that any such transformation can be exponentiated to yield a representation of the corresponding Weyl algebra over $(H, \sigma)$, and that the transformation produces a representation of the CCR unitarily equivalent with the original Fock representation if and only if $\sum_{k, l, m} \lambda_{k l m}^{2}<\infty$. As we shall see below, this condition can be equivalently expressed in terms of a certain operator being Hilbert-Schmidt.

The paper is organized as follows. After establishing the notation and general setting in Chapter II, quadratic transformations are defined and studied in Chapter III, where the canonical quadratic transformations are explicitly characterized. In Chapter IV it is shown that any canonical quadratic transformation may be exponentiated, and the mentioned necessary and sufficient conditions for their unitary implementability are proven. Finally, further developments, speculations and possible applications are discussed in Chapter $V$.

Some of the results presented in this paper have been announced in [22].

\section{Notation and General Setting}

For us the basic starting point is a real (nondegenerate) symplectic space $(H, \sigma)$ with an associated (regular) Weyl system $(\mathscr{K}, W(f))$ consisting of a complex Hilbert space $\mathscr{K}$ and a mapping $W: H \mapsto \mathcal{U}(\mathscr{K})$ from $H$ into the group $\mathcal{U}(\mathscr{K})$ of unitary operators on $\mathscr{K}$ which satisfies the following axioms [13]:

\footnotetext{
${ }^{1}$ In the case (1.2), the transformation is called quadratic; for a brief discussion of transformations of degree greater than 2 , see Chapter 5 and [22].

${ }^{2}$ which we call standard
} 


$$
\begin{gathered}
W(f) W(g)=e^{-i \sigma(f, g) / 2} W(f+g), \quad \forall f, g \in H, \\
W(f)^{*}=W(-f), \quad \forall f \in H,
\end{gathered}
$$

and

$$
\mathbf{R} \ni t \mapsto W(t f) \in \mathscr{B}(\mathscr{K}) \text { is weakly continuous for all } f \in H \text {. }
$$

$(\mathscr{B}(\mathscr{K})$ denotes the set of all bounded linear operators $A: \mathscr{K} \mapsto \mathscr{K}$.) Condition (2.3) entails that the map $\mathbf{R} \ni t \mapsto W(t f) \in \mathscr{B}(\mathscr{K})$ is actually strongly continuous, hence by Stone's Theorem one knows that for each $f \in H$ there exists a self-adjoint operator $\Phi(f)$ on $\mathscr{K}$ such that $W(t f)=e^{i t \Phi(f)}$ for all $t \in \mathbf{R}$ and by (2.1) the map $f \mapsto \Phi(f)$ is (real) linear. In fact, there exists a dense domain of vectors $D_{W} \subset \mathcal{K}$ which is a core of and left invariant by every $\Phi(f)$ [19] [10]; it is on this domain that the linearity just mentioned can be verified. On this domain one also verifies that the generators satisfy the CCR:

$$
\Phi(f) \Phi(g)-\Phi(g) \Phi(f)=i \sigma(f, g) \mathbf{1}, \forall f, g \in H
$$

We therefore also call the Weyl system $(\mathscr{K}, W(f))$ and its associated generators as above a regular representation of the CCR over $(H, \sigma)$.

We shall denote by $\mathscr{A}(H, \sigma)$ the $C^{*}$-algebra on $\mathscr{K}$ generated by the operators $\{W(f) \mid f \in H\}$. As the notation indicates, the algebra $\mathscr{A}(H, \sigma)$ does not depend on the choice of representation of the Weyl operators $\{W(f) \mid f \in H\}[28]$ [2, Theorem 5.2.8]. $\mathscr{A}(H, \sigma)$ is a simple, nonseparable $C^{*}$-algebra [28] [13]. There are, in fact, many different $C^{*}$-algebras one can associate with the CCR (see [11] for a discussion of some of the alternatives), and the one we have chosen is minimal in the sense of set containment [15]; but, for practical purposes the choice is moot, since one is generally interested in a von Neumann algebra which is 'generated' by the $C^{*}$-algebra, and all the $C^{*}$-algebras discussed in [11] (realized concretely on a given representation space) have the same weak closure.

For any real linear map $J: H \mapsto H$ satisfying $\sigma(J f, J g)=\sigma(f, g),-\sigma(J f, f)>$ $0(f \neq 0)$, and $J^{2}=-1$, one can introduce a complex structure on $H$ as follows ${ }^{3}$ [11] : $(\alpha+i \beta) f \equiv \alpha f+\beta J f$, for all $f \in H$, and $\alpha, \beta \in \mathbf{R}$. Moreover, $\langle f, g\rangle_{\mathscr{H}} \equiv$ $-\sigma(J f, g)+i \sigma(f, g)$ defines a scalar product on $H$ such that $\left(H,\langle\cdot, \cdot\rangle_{\mathscr{H}}\right)$ is a complex preHilbert space [11]. If on the other hand one begins with a complex Hilbert space $\mathscr{H}$ with scalar product $\langle\cdot, \cdot\rangle_{\mathscr{H}}$, then with $\sigma(f, g)=\mathfrak{m}\langle f, g\rangle_{\mathscr{H}}$, $(H, \sigma)$ is a real symplectic space of the sort with which we began, and with

\footnotetext{
${ }^{3}$ It should be mentioned that such a $J$ does not necessarily exist for arbitrary choice of symplectic space $(H, \sigma)$.
} 
$\langle f, g\rangle \equiv \Re e\langle f, g\rangle_{\mathscr{H}}$, then $(H,\langle\cdot, \cdot\rangle)$ is a real Hilbert space.

With a choice of a $\sigma$-admissible complex structure $J$ on $(H, \sigma)$, there is an important representation of $\mathscr{A}(H, \sigma)$ called the Fock representation. This is given as the GNS-representation $\{\mathscr{K}, \pi, \Omega\}$ associated with the state [14] $\omega_{J}$ determined on $\mathscr{A}(H, \sigma)$ by

$$
\omega_{J}(W(f)) \equiv e^{\sigma(J f, f) / 4}
$$

Given such a representation, one can define the following 'annihilation' and 'creation' operators:

$$
a(f) \equiv \frac{1}{\sqrt{2}}(\Phi(f)+i \Phi(J f)), \quad a^{\dagger}(f) \equiv \frac{1}{\sqrt{2}}(\Phi(f)-i \Phi(J f)),
$$

where $\pi(W(t f))=e^{\imath t \Phi(f)}$. One has then $a(f) \Omega=0$ for all $f \in H$.

For the purposes of this paper, we shall assume that a choice of $\sigma$-admissible complex structure J has been made on $(H, \sigma)$, so we have the complex one-particle space $\mathscr{H}$ and a corresponding Fock representation. Since as sets $H=\mathscr{H}$, we shall distinguish notationally the vector $f$ viewed as an element of the real Hilbert space $H$ from the same vector, denoted as $\widetilde{f}$, viewed as an element of the complex Hilbert space $\mathscr{H}$. If $\left\{\tilde{e}_{k}\right\}_{k \in \mathbf{N}}$ forms an orthonormal basis in $\mathscr{H}$, then with $J e_{k} \equiv e_{k^{\prime}}$, the set $\left\{e_{K}\right\}=\left\{e_{k}, e_{k^{\prime}}\right\}=\left\{e_{k}, J e_{k}\right\}$ (in this paper, large-lettered indices $K, L, M, \ldots$ are understood to run through the set $\left\{1,1^{\prime}, 2\right.$, $\left.2^{\prime}, \ldots\right\}$, whereas small-lettered indices $k, l, m, \ldots$ run through $\left.\{1,2,3, \ldots\} \equiv \mathbf{N}\right)$ forms a symplectic orthonormal system in $H$, in particular

$$
\begin{gathered}
\sigma\left(e_{k}, e_{l}\right)=\sigma\left(e_{k^{\prime}}, e_{l^{\prime}}\right)=0, \quad \sigma\left(e_{k}, e_{l^{\prime}}\right)=\delta_{k l}, \\
\left\langle e_{k}, e_{l}\right\rangle=\left\langle e_{k^{\prime}}, e_{l^{\prime}}\right\rangle=\delta_{k l}, \quad\left\langle e_{k}, e_{l^{\prime}}\right\rangle=0,
\end{gathered}
$$

where in our notation the last line can be written as $\left\langle e_{K}, e_{L}\right\rangle=\delta_{K L}$.

With the choice of complex structure made as above, the corresponding Fock state denoted by $\omega_{F}: \mathscr{A}(H, \sigma) \mapsto \mathbf{C}$ now satisfies

$$
\omega_{F}(W(f))=e^{-\|f\|^{2 / 4}},
$$

and the associated GNS-space may be represented by the symmetric Fock space $\mathscr{F}_{+}(\mathscr{H})$. We recall that the Fock space $\mathscr{F}(\mathscr{H})=\oplus_{n=0}^{\infty} \mathscr{H}^{n}\left(\mathscr{H}^{0}=\mathbb{C}, \mathscr{H}^{n}\right.$ the $n$-fold tensor product of $\mathscr{H}$ with itself), and that $\mathscr{F}_{+}(\mathscr{H})$ is the totally symmetric subspace $\oplus_{n=0}^{\infty} P_{+} \mathscr{H}^{n}$ of $\mathscr{F}(\mathscr{H})$, where $P_{+}$is the projection 


$$
P_{+}\left(\widetilde{f}_{1} \otimes \widetilde{f}_{2} \otimes \cdots \otimes \widetilde{f_{n}}\right)=\frac{1}{n !} \sum_{\pi} \widetilde{f}_{\pi(1)} \otimes \widetilde{f}_{\pi(2)} \otimes \cdots \otimes \widetilde{f}_{\pi(n)}
$$

$\left(\widetilde{f}_{\imath} \in \mathscr{H}, \pi\right.$ : permutation on the set $\left.\{1,2, \ldots, n\}\right)$. The projection operator $P_{n}:$ $\mathscr{F}(\mathscr{H}) \mapsto \mathscr{H}^{n}$ projects onto the $n$-particle subspace, and we shall write $\varphi^{(n)} \equiv P_{n} \varphi$ as the $n$-particle component of $\varphi \in \mathscr{F}(\mathscr{H})$. The vector $\Omega_{0} \equiv(1,0,0, \ldots) \in \mathscr{F}_{+}(\mathscr{H})$ is the Fock vacuum. $\mathscr{F}_{0}$ is the finite-particle subspace, i.e. the linear span of the ranges of $\left\{P_{n}\right\}_{n \in \mathbf{N}}$. The GNS-representation for the Fock state may be identified with $\left\{\mathscr{F}_{+}(\mathscr{H}), \pi_{F}, \Omega_{0}\right\}$.

With $a(\widetilde{f})$ the usual annihilation operator in $\mathscr{F}_{+}(\mathscr{H})$ for $\widetilde{f} \in \mathscr{H}$ and the adjoint operator $a(\widetilde{f})^{*}$ the corresponding creation operator, then the linear selfadjoint operator $\Phi_{s}(\widetilde{f}) \equiv \frac{1}{\sqrt{2}} \overline{\left(a(\widetilde{f})+a(\widetilde{f})^{*}\right)}$ (the bar denotes the closure of the operator) is called the Segal field operator in $\mathscr{F}_{+}(\mathscr{H})$. If we also view $\widetilde{f} \in \mathscr{H}$ as an element of $H$, then we have $\Phi_{s}(\widetilde{f})=\Phi(f)$, after identifying $\mathscr{A}(H, \sigma)$ with $\pi_{F}\left(\mathscr{A}(H, \sigma)\right.$ ) (since they are isomorphic). $\mathscr{F}_{0}$ is a core for $\Phi(f)$, and for $\varphi \in \mathscr{F}_{0}$, $\Phi(f)$ satisfies the bound $\|\Phi(f) \varphi\| \leq \sqrt{2} \sqrt{n_{\varphi}+1}\|f\|\|\varphi\|$, where $n_{\varphi}$ equals the smallest $n \in \mathbf{N}$ such that $P_{N} \varphi=0$ for all $N>n$. If $\left(f_{n}\right)_{n=1}^{\infty}$ converges in $H$ to $f$, then for every $\varphi \in \mathscr{F}_{0}$ the sequence $\left(\Phi\left(f_{n}\right) \varphi\right)_{n=1}^{\infty}$ converges in $\mathscr{F}_{+}(\mathscr{H})$ to $\Phi(f) \varphi$. To make a notational connection to the discussion in Section I and in keeping with common harmonic oscillator conventions, if $\left\{e_{K}\right\}$ is a symplectic orthonormal basis in $H$, then the 'position operator' and the 'momentum operator' corresponding to the $k$-th degree of freedom are given by

$$
q_{k} \equiv \Phi_{s}\left(\widetilde{e_{k}}\right)=\Phi\left(e_{k}\right), \quad p_{k} \equiv \Phi_{s}\left(i \widetilde{e_{k}}\right)=\Phi\left(J e_{k}\right)=\Phi\left(e_{k^{\prime}}\right)
$$

\section{Quadratic Transformations}

In this chapter we rigorously define what we mean by quadratic transformations and then explicitly characterize those which are canonical, i.e. those which lead to a representation of the CCR. We shall show that any such transformation can be written in the standard form of equation (1.2). In the following, $D(O)$ (resp. $R(O)$ ) will denotes the domain (resp. range) of the operator $O$.

\section{§3.1. Two-Particle Operators and Generators}

We first define and study the two-particle operators and their associated transformations. Let $F \in H \odot H$ have the form $F=\sum_{i=1}^{n} \alpha_{i}\left(f_{i} \otimes g_{i}\right)$, with $\alpha_{i} \in \mathbf{R}, f_{i}, g_{i}$ 
$\in H$ and $n \in \mathbf{N}$. We define an operator $\Psi(F)$ acting on the domain $\mathscr{F}_{0} \subset \mathscr{F}_{+}(\mathscr{H})$ by

$$
\Psi(F) \phi=\Psi\left(\sum_{i=1}^{n} \alpha_{i}\left(f_{i} \otimes g_{i}\right)\right) \phi \equiv \sum_{i=1}^{n} \alpha_{i}: \Phi\left(f_{i}\right) \Phi\left(g_{i}\right): \phi, \quad \phi \in \mathscr{F}_{0}
$$

where the Wick ordering is taken with respect to the Fock vacuum $\Omega_{0}$, i.e.

$$
: \Phi(f) \Phi(g):=\Phi_{s}(\widetilde{f}) \Phi_{s}(\widetilde{g})-\frac{1}{2}\langle\widetilde{f}, \widetilde{g}\rangle_{\mathscr{H}} \mathbb{1}=\Phi(f) \Phi(g)-\frac{1}{2}\langle f, g\rangle \mathbb{1}-\frac{i}{2} \sigma(f, g) \mathbb{1}
$$

It is easy to verify that this definition is independent of the special choice of linear representation for $F$ (see [18], pp. 14-15).

Lemma 3.1.1. For each $\phi \in \mathscr{F}_{0}$ the mapping $F \mapsto \Psi(F) \psi$ from $H \odot H$ to $\mathscr{F}_{+}(\mathscr{H})$ is bounded with

$$
\|\Psi(F) \phi\| \leq 2 \sqrt{2} \sqrt{\left(n_{\psi}+2\right)\left(n_{\psi}+1\right)}\|F\|\|\varphi\|
$$

for any $\phi \in \mathscr{F}_{0}$. Hence it may be extended uniquely to a continuous linear map from $H$ $\otimes H\left(\equiv H^{2}\right)$ to $\mathscr{F}_{+}(\mathscr{H})$. Consequently, for each $F \in H^{2}$ there exists a linear operator with domain $\mathscr{F}_{0}$, which shall again be denoted by $\Psi(F)$ and which satisfies the same bound (3.1.1).

Proof. 1. With $F=\sum_{i=1}^{n} \alpha_{i}\left(f_{\imath} \otimes g_{i}\right)$, there is a finite orthonormal and symplectic system $\left\{e_{1}, J e_{1}, \ldots, e_{m}, J e_{m}\right\}=\left\{e_{K} \mid K=1,1^{\prime}, \ldots, m, m^{\prime}\right\}=\left\{e_{K}\right\}_{K \in M}$, with $M=\left\{1,1^{\prime}\right.$, $\left.\ldots, m, m^{\prime}\right\}, 2 m \leq n$, such that $F=\sum_{K, L \in \mathcal{M}} \beta_{K L}\left(e_{K} \otimes e_{L}\right)$. This can be seen by considering the subspace of $\mathscr{H}$ spanned by $\widetilde{f}_{1}, \ldots, \widetilde{f_{n}}, \widetilde{g_{1}}, \ldots, \widetilde{g_{n}}$ and choosing an orthonormal basis $\left\{h_{1}, \ldots, h_{m}\right\}$ for it. $\left\{e_{i}\right\}$ will denote the corresponding vectors in $H$, i.e. $\widetilde{e_{i}}=h_{i}$. Then one has $\widetilde{f_{1}}=\sum_{i=1}^{m} \gamma_{i} \widetilde{e_{i}}$ if and only if $f_{1}=\sum_{i=1}^{m} \Re e \gamma_{i} e_{i}+\Im m \gamma_{i} J e_{i}$ (similarly for the other test functions). With $F$ now expressed as $F=\sum_{K, L \in M} \beta_{K L}\left(e_{K} \otimes e_{L}\right)$, define $\widetilde{F}=\sum_{K, L \in M} \beta_{K L}\left(\widetilde{e_{K}} \otimes \widetilde{e_{L}}\right) \in \mathscr{H}^{2}$. Then one has $\|\widetilde{F}\| \leq \sqrt{2}\|F\|$, which can be verified by direct computation.

2. One then computes:

$$
\Psi(F) \phi=\sum_{K, L \in \mathcal{M}} \beta_{K L}: \Phi\left(e_{K}\right) \Phi\left(e_{L}\right): \psi
$$




$$
\begin{aligned}
= & \frac{1}{2} \sum_{K, L \in M} \beta_{K L}\left\{a^{*}\left(\widetilde{e_{K}}\right) a^{*}\left(\widetilde{e_{L}}\right)+a\left(\widetilde{e_{K}}\right) a\left(\widetilde{e_{L}}\right)+a^{*}\left(\widetilde{e_{K}}\right) a\left(\widetilde{e_{L}}\right)+a^{*}\left(\widetilde{e_{L}}\right) a\left(\widetilde{e_{K}}\right)\right\} \phi ; \\
\|\Psi(F) \phi\| \leq & \frac{1}{2}\left\{\left\|\sum_{K, L M M} \beta_{K L} a^{*}\left(\widetilde{e_{K}}\right) a^{*}\left(\widetilde{e_{L}}\right) \phi\right\|+\left\|\sum_{K, \in M} \beta_{K L} a\left(\widetilde{e_{K}}\right) a\left(\widetilde{e_{L}}\right) \phi\right\|\right. \\
& \left.+\left\|\sum_{K, L \in M} \beta_{K L} a^{*}\left(\widetilde{e_{K}}\right) a\left(\widetilde{e_{L}}\right) \phi\right\|+\left\|\sum_{K, L \in M}^{K L} \beta_{K L} a^{*}\left(\widetilde{e_{L}}\right) a\left(\widetilde{e_{K}}\right) \phi\right\|\right\} .
\end{aligned}
$$

3. Now, in particular, one has

$$
\begin{aligned}
\left\|\sum_{K L \in M} \beta_{K L} a^{*}\left(\widetilde{e_{K}}\right) a^{*}\left(\widetilde{e_{L}}\right) \phi\right\|^{2} & =\sum_{n=0}^{n_{\psi}}\left\|\sum_{K L \in \mathcal{M}} \beta_{K L} a^{*}\left(\widetilde{e_{K}}\right) a^{*}\left(\widetilde{e_{L}}\right) \phi^{(n)}\right\|^{2} \\
& =\sum_{n=0}^{n_{\psi}}\left\|\sum_{K, L \in M} \beta_{K L} \sqrt{n+2} \sqrt{n+1} P_{+} \widetilde{e_{K}} \otimes \widetilde{e_{L}} \otimes \phi^{(n)}\right\|^{2} \\
& =\sum_{n=0}^{n_{\psi}}(n+2)(n+1)\left\|P+\tilde{F} \otimes \phi^{(n)}\right\|^{2} \\
& \leq\left(n_{\psi}+2\right)\left(n_{\psi}+1\right)\|\tilde{F}\|^{2} \sum_{n=0}^{n_{\psi}}\left\|\phi^{(n)}\right\|^{2} \\
& \leq 2\left(n_{\psi}+2\right)\left(n_{\psi}+1\right)\|F\|^{2}\|\phi\|^{2} .
\end{aligned}
$$

Thus, the map $\phi^{(n)} \mapsto a^{*}\left(\widetilde{e_{K}}\right) a^{*}\left(\widetilde{e_{L}}\right) \phi^{(n)}$ is a bounded linear map from $P_{+} \mathscr{H}^{n}$ to $P_{+} \mathscr{H}^{(n+2)}$ with norm bounded by $\sqrt{2} \sqrt{(n+2)(n+1)}\|F\|$. The map $\phi^{(n+2)} \mapsto$ $a\left(\widetilde{e}_{K}\right) a\left(\widetilde{e}_{L}\right) \phi^{(n+2)}$ is (a restriction of) its adjoint and therefore satisfies the bound: $\left\|\sum_{K, L \in \mathcal{M}} \beta_{K L} a\left(\widetilde{e}_{K}\right) a\left(\widetilde{e}_{L}\right) \phi^{(n+2)}\right\| \leq \sqrt{2} \sqrt{(n+2)(n+1)}\|F\|\left\|\phi^{(n+2)}\right\|$, which entails $\left\|\sum_{K, L \in \mathcal{M}} \beta_{K L} a\left(\widetilde{e_{K}}\right) a\left(\widetilde{e_{L}}\right) \phi\right\|^{2} \leq 2\left(n_{\psi}+2\right)\left(n_{\psi}+1\right)\|F\|^{2}\|\phi\|^{2}$.

4. Similarly, one finds

$$
\begin{aligned}
\sum_{K, L \in \mathcal{M}} \beta_{K L} a^{*}\left(\widetilde{e_{K}}\right) a\left(\widetilde{e_{L}}\right) \phi^{(n)} & =\sum_{k=1}^{m} \sum_{L \in \mathcal{M}}\left(\beta_{k L}+i \beta_{k^{\prime} L}\right) a^{*}\left(\widetilde{e_{k}}\right) a\left(\widetilde{e_{L}}\right) \phi^{(n)} \\
& =\sum_{k=1}^{m} \sqrt{n} P_{+} \widetilde{e_{k}} \otimes\left[a\left(\sum_{L \in \mathcal{M}}\left(\beta_{k L}-i \beta_{k^{\prime} L}\right) \widetilde{e_{L}}\right) \psi^{(n)}\right] .
\end{aligned}
$$

In light of the standard bound $\left\|a(f) \phi^{(n)}\right\| \leq \sqrt{n}\|f\|\left\|\phi^{(n)}\right\|$, it now follows that

$$
\begin{aligned}
\left\|\sum_{K L \in \mathcal{M}} \beta_{K L} a^{*}\left(\widetilde{e_{K}}\right) a\left(\widetilde{e_{L}}\right) \phi^{(n)}\right\|^{2} & \leq n \sum_{k=1}^{m} n\left\|\sum_{L \in \mathcal{M}}\left(\beta_{k L}+i \beta_{k^{\prime} L}\right) \widetilde{e_{L}}\right\|^{2}\left\|\phi^{(n)}\right\|^{2} \\
& \leq 2 n^{2}\|F\|\left\|\phi^{(n)}\right\|^{2} .
\end{aligned}
$$

Thus one has the inequality $\left\|\sum_{K, L \in M} \beta_{K L} a^{*}\left({\widetilde{e_{K}}}\right) a\left(\widetilde{e_{L}}\right) \phi\right\|^{2} \leq 2\left(n_{\psi}+2\right)\left(n_{\psi}+1\right)\|F\|^{2}\|\phi\|^{2}$.

The same bound is applicable to the remaining term in (3.1.2).

Putting together Steps (2)-(4), one may conclude 


$$
\|\Psi(F) \phi\| \leq \frac{1}{2} \cdot 4 \sqrt{2} \sqrt{\left(n_{\psi}+2\right)\left(n_{\psi}+2\right)}\|F\|\|\phi\| .
$$

Of course, a consequence of this lemma is that if $\left\{F_{n}\right\} \subset H^{2}$ converges to $F$ $\in H^{2}$, then the operators $\Psi\left(F_{n}\right)$ converge strongly to $\Psi(F)$ on $\mathscr{F}_{0}$. This would not be true if in the definition of the two particle operators $\Psi(F)$ normal ordering had not been used (see [18], p.20). Another technically important fact is also implied by the previous lemma.

Corollary 3.1.2. For any $F \in H^{2}, \Psi(F)$ is a symmetric operator for which the equality $\Psi(F)=\Psi\left(P_{+} F\right)+\Psi\left(P_{-} F\right)=\Psi\left(P_{+} F\right)$ obtains.

Proof. That $\Psi(F){ }^{*} \psi=\Psi(F) \psi$ for $F \in H \odot H$ and $\phi \in \mathscr{F}_{0}$ is obvious. The continuity property established in Lemma 3.1.1 then entails that the operator $\Psi(F)$ is symmetric on $\mathscr{F}_{0}$ for all $F \in H^{2}$.

Moreover, since $\Psi(f \otimes g)=\Psi(g \otimes f)$, the equality $\Psi(F)=\Psi\left(P_{+} F\right)$ follows for $F \in H \odot H$ and once again by continuity for $F \in H^{2}$.

Therefore, with $f \in H$ and $F \in H^{2}$, we have $(\Phi(f)+\Psi(F)) * \supset \Phi(f)+\Psi(F) *$ $\supset \Phi(f)+\Psi(F)$, so that the operator $\Phi(f)+\Psi(F)$ is symmetric and closable. If $\Lambda: H \mapsto P_{+} H^{2}$ is a linear, densely defined operator, then we define a quadratic transformation of the field to be

$$
\Phi(f) \mapsto \Phi_{\Lambda}(f) \equiv \overline{\Phi(f)+\Psi(\Lambda f)}, \quad f \in D(\Lambda)
$$

where $\overline{\Phi(f)+\Psi(\Lambda f)}$ denotes the closure of $\Phi(f)+\Psi(\Lambda f)$ on $\mathscr{F}_{0}$. It is easy to verify that $\Phi_{\Lambda}(f)=\overline{\left.\Phi(f)\right|_{\mathscr{F}_{0}}+\Psi(\Lambda f)}$ and $\Phi_{\Lambda}(f)=\overline{\Phi(f)+\overline{\Psi(\Lambda f)}}$ (see [18], Satz 3.1). We next shall see that $\Phi_{\Lambda}(f)$ is self-adjoint.

Proposition 3.1.3. For every $f \in H$ and $F \in H^{2}$ the operator $\Phi(f)+\Psi(F)$ is essentially self-adjoint (on $\mathscr{F}_{0}$ ). In particular, for every $f \in D(\Lambda)$ the operator $\Phi_{\Lambda}(f)$ $\equiv \overline{\Phi(f)+\Psi(\Lambda f)}$ is self-adjoint.

Proof. Let $\phi$ be a finite particle vector, i.e. let $\phi=\left(\phi^{(n)}\right)_{n=0}^{\infty}, \psi^{(n)} \in P_{+} \mathscr{H}^{n}$, and $\psi^{(n)}=0$ for all $n>n_{\psi}$. As a shorthand we write: $\widehat{\Phi} \equiv \Phi(f)+\Psi(F)$ for arbitrary fixed $f \in H$ and $F \in H^{2}$, as well as $\alpha \equiv\|f\|+2\|F\|$. Since $\widehat{\Phi}$ maps every finite particle vector onto a vector in $\mathscr{F}_{0}$, one has

$$
\phi \in C^{\infty}(\widehat{\Phi}) \equiv \cap_{k=1}^{\infty} D\left(\widehat{\Phi}^{k}\right)
$$


Furthermore, one has

$$
\begin{aligned}
\|\widehat{\Phi} \phi\| & =\|\Phi(f) \phi+\Psi(F) \phi\| \leq\|\Phi(f) \phi\|+\|\Psi(F) \phi\| \\
& \leq \sqrt{2} \sqrt{n_{\psi}+1}\|f\|\|\psi\|+2 \sqrt{2} \sqrt{\left(n_{\psi}+1\right)\left(n_{\psi}+2\right)}\|F\|\|\phi\| .
\end{aligned}
$$

For the first term of the last line see e.g. the proof of Theorem X.41 in [21]; for the second term see the proof of Lemma 3.1.1 above. Therefore one has

$$
\|\widehat{\Phi} \phi\| \leq \sqrt{2} \sqrt{\left(n_{\psi}+1\right)\left(n_{\psi}+2\right)}(\|f\|+2\|F\|)\|\phi\|=\sqrt{2} \alpha \sqrt{\left(n_{\psi}+1\right)\left(n_{\psi}+2\right)}\|\phi\| .
$$

In light of the fact that the application of $\widehat{\Phi}$ to a finite particle vector increases the highest 'particle-number' at most by 2 (since it contains two creation operators), one concludes that

$$
\begin{aligned}
\left\|\widehat{\Phi}^{n} \phi\right\| & \leq \sqrt{2} \alpha\left[\left(n_{\psi}+2(n-1)+1\right)\left(n_{\psi}+2(n-1)+2\right)\right]^{1 / 2}\left\|\widehat{\Phi}^{n-1} \phi\right\| \\
& \leq 2^{n / 2} \alpha^{n}\left[\left(n_{\psi}+1\right)\left(n_{\psi}+2\right) \cdots\left(n_{\psi}+2 n\right)\right]^{1 / 2}\|\phi\| \\
& \leq 2^{n / 2} \alpha^{n} \sqrt{\left(n_{\psi}+2 n\right) !}\|\phi\| .
\end{aligned}
$$

In order to obtain a sufficient condition for the convergence of the series $\sum_{n=0}^{\infty} \frac{1}{n !}\left\|\widehat{\Phi}^{n} \psi\right\| t^{n}(t>0)$, one can apply the ratio test to its majorant

$\sum_{n=0}^{\infty} \frac{1}{n !} 2^{n / 2} \alpha^{n} t^{n} \sqrt{\left(n_{\psi}+2 n\right) !}\|\phi\|$ to find that

$$
\begin{aligned}
\frac{\left(2^{n / 2} / n !\right) \alpha^{n} t^{n} \sqrt{\left(n_{\psi}+2 n\right) !}}{\left(2^{(n-1) / 2} /(n-1) !\right) \alpha^{n-1} t^{n-1} \sqrt{\left(n_{\psi}+2(n-1)\right) !}} & =\frac{\sqrt{2}}{n} \alpha t \sqrt{\left(n_{\psi}+2 n-1\right)\left(n_{\psi}+2 n\right)} \\
& \leq \frac{\sqrt{2}}{n} \alpha t\left(n_{\psi}+2 n\right) \underset{n \rightarrow \infty}{\rightarrow} 2 \sqrt{2} \alpha t .
\end{aligned}
$$

Thus one has $\sum_{n=0}^{\infty} \frac{1}{n !}\left\|\widehat{\Phi}^{n} \psi\right\| t^{n}<\infty$ for $t<\frac{1}{2 \sqrt{2} \alpha}$, so $\phi$ is an analytic vector for $\widehat{\Phi}$. Since therefore all $\phi \in \mathscr{F}_{0}$ are analytic vectors for $\widehat{\Phi}$, since $\mathscr{F}_{0}$ is dense in Fock space and left invariant by $\widehat{\Phi}$, and since $\widehat{\Phi}$ is symmetric, the essential selfadjointness of $\widehat{\Phi}$ follows from Nelson's Theorem (see e.g. [21], Theorem X.6).

Remarks. 1. Also the operator $\Psi(F)$ is essentially self-adjoint, as one sees by setting $f=0$. The proof cannot be applied to an $N$-particle operator $\Psi^{(N)}(F)$, $F \in H^{N}$ with $N>2$, since then $\sqrt{\left(n_{\psi}+2 n\right) !}$ becomes $\sqrt{\left(n_{\psi}+N n\right) !}$, and the ratio 
test no longer yields convergence.

2. Because of the essential self-adjointness of $\Psi(F)$ and the proven continuity property, one may conclude from standard theorems that if $\left\{F_{n}\right\} \subset H^{2}$ converges to $F \in H^{2}$, then the operators $\overline{\Psi\left(F_{n}\right)}$ converge to $\overline{\Psi(F)}$ in the strong resolvent sense, and $\overline{\Psi(F)}$ is the strong graph limit of $\left\{\overline{\Psi\left(F_{n}\right)}\right\}$ (see, e.g., [20], Theorems VIII.25 and VIII.26).

\section{§3.2. Canonical Quadratic Transformations}

In this section we determine necessary and sufficient conditions on the linear, densely defined map $\Lambda: H \mapsto H^{2} \equiv H \otimes H$ such that the quadratic field transformation

$$
\Phi(f) \mapsto \Phi_{\Lambda}(f) \equiv \overline{\Phi(f)+\Psi(\Lambda F)}, \quad f \in D(\Lambda),
$$

is canonical. In light of Proposition 3.1.3, for every $f \in D(\Lambda)$ the transformed field operator $\Phi_{\Lambda}(f)$ is self-adjoint. We assume $\Lambda$ to be linear, so that $\Phi_{\Lambda}(f) \varphi$ is linear in $f$, analogously to $\Phi(f) \varphi$ (for $\varphi \in \mathscr{F}_{0}$ ). Moreover, $D(\Lambda)$ should be dense, so that there exists no nonzero $f \perp D(\Lambda)$, i.e. no degree of freedom, for which the transformation (3.2.1) would not be defined. On the other hand, $D(\Lambda)=H$ is not required, as we wish to admit unbounded $\Lambda$. Due to Corollary 3.1 .2 , the range $R(\Lambda)$ of $\Lambda$ may be taken to lie in $P_{+} H^{2}$ without loss of generality. For convenience, we introduce the following definition.

Definition 3.2.1. Let $\mathscr{L}$ be the set of all linear, densely defined operators from $H$ to $P_{+} H^{2}$, and let $\mathscr{L}_{C C R} \subset \mathscr{L}$ be the subset of $\mathscr{L}$ consisting of elements $\Lambda$ such that the transformation $\Phi(f) \mapsto \Phi_{\Lambda}(f)=\overline{\Phi(f)+\Psi(\Lambda f)}, f \in D(\Lambda)$, is canonical, i.e. one has

$$
\left[\Phi_{\Lambda}(f), \Phi_{\Lambda}(g)\right] \subset i \sigma(f, g) \mathbb{1}
$$

whenever $f, g \in D(\Lambda)$.

Note that since $\mathscr{F}_{0}$ is an invariant core for $\Phi_{\Lambda}(f)$, it suffices to verify equation (3.2.2) on the elements of $\mathscr{F}_{0}$. We introduce now a symplectic bilinear form on $H^{2}$.

Definition 3.2.2. For $F, G \in H^{2}$ let $\sigma(F, G) \equiv\langle(J \otimes \mathbb{1}) F, G\rangle$.

The map $\sigma: H^{2} \times H^{2} \mapsto \mathbf{R}$ is bilinear and alternating, since $J \otimes \mathbb{1}$ is unitary 
and we have

$$
\begin{aligned}
\sigma(F, G) & =\langle(J \otimes \mathbf{1})(J \otimes \mathbf{1}) F,(J \otimes \mathbf{1}) G\rangle \\
& =\langle(-\mathbf{1} \otimes \mathbf{1}) F,(J \otimes \mathbf{1}) G\rangle=-\langle(J \otimes \mathbf{1}) G, F\rangle=-\sigma(G, F) .
\end{aligned}
$$

In addition, this form is nondegenerate, since if $\sigma(F, G)=0$ for all $F \in H^{2}$, it follows that

$$
0=\sigma(-(J \otimes 1) G, G)=-\left\langle(J \times 1)^{2} G, G\right)=\langle G, G\rangle=\|G\|^{2},
$$

which entails $G=0$. We remark that for simple tensors $f_{1} \otimes f_{2}, g_{1} \otimes g_{2} \in H^{2}$, one has

$$
\sigma\left(f_{1} \otimes f_{2}, g_{1} \otimes g_{2}\right)=\left\langle\left(J f_{1}\right) \otimes f_{2}, g_{1} \otimes g_{2}\right\rangle=\left\langle J f_{1}, g_{1}\right\rangle\left\langle f_{2}, g_{2}\right\rangle=\sigma\left(f_{1}, g_{1}\right)\left\langle f_{2}, g_{2}\right\rangle,
$$

where the final $\sigma$ naturally is the symplectic form on $H$.

Definition 3.2.3. For any $f \in H$ and $F \in H^{2}$, let $b(f, F)$ denote the element of $H$ such that

$$
\langle b(f, F), g\rangle=\sigma(f \otimes g, F)
$$

holds for all $g \in H$.

$b$ is a well-defined map from $H \times H^{2}$ into $H$, as can be seen as follows. Consider the map

$$
g \mapsto \sigma(f \otimes g, F), g \in H,
$$

which is a bounded linear functional on $H$, since

$$
|\sigma(f \otimes g, F)|=|\langle(J f) \otimes g, F\rangle| \leq\|(J f) \otimes g\|\|F\|=\|J f\|\|g\|\|F\|=\|f\|\|F\|\|g\| .
$$

Therefore, according to the Riesz representation theorem there exists exactly one vector in $H$, which we shall denote by $b(f, F)$, so that equation (3.2.4) holds for all $g \in H$. In addition, Riesz' theorem entails that the norm of $b(f, F)$ is equal to that of the map in (3.2.5), hence we have $\|b(f, F)\| \leq\|f\|\|F\|$. Thus $b$ is linear and continuous in each entry, and for $F=f_{1} \otimes f_{2}$ one sees that

$$
\left\langle b\left(f, f_{1} \otimes f_{2}\right), g\right\rangle=\sigma\left(f \otimes g, f_{1} \otimes f_{2}\right)=\sigma\left(f, f_{1}\right)\left\langle g, f_{2}\right\rangle=\left\langle\sigma\left(f, f_{1}\right) f_{2}, g\right\rangle .
$$


Since this obtains for all $g \in H$, one has

$$
b\left(f, f_{1} \otimes f_{2}\right)=\sigma\left(f, f_{1}\right) f_{2} .
$$

Thus, we could have defined $b$ via linear and then continuous extension of (3.2.6). We define next a related map $B$.

Definition 3.2.4. For any $F, G \in H^{2}$, let $B(F, G)$ denote that element of $H^{2}$ for which $b(h, B(F, G))=b(b(h, F), G)$ holds for all $h \in H$.

Lemma 3.2.5. $\quad B$ is a well-defined bounded linear map from $H^{2} \times H^{2}$ into $H^{2}$.

Proof. 1. Let $\left\{e_{s}\right\}$ be an orthonormal basis in $H$ and fix $F, G \in H^{2}$. Then $\left\{J e_{s}\right\}$ is also an orthonormal basis, and one has

$$
F=\sum_{r, s} \alpha_{r s}\left(J e_{r}\right) \otimes\left(J e_{s}\right)=\sum_{r}\left(J e_{r}\right) \otimes f_{r}
$$

with $\alpha_{r s} \in \mathbf{R}$ and $f_{r} \equiv \sum_{s} \alpha_{r s} J e_{s}$. Define furthermore the vectors $B_{s} \in H^{2}, B_{s} \equiv\left(J e_{s}\right)$ $\otimes b\left(b\left(e_{s}, F\right), G\right)$, which are mutually orthogonal and whose norms satisfy

$$
\begin{aligned}
\left\|B_{s}\right\| & =\left\|b\left(b\left(e_{s}, F\right), G\right)\right\| \leq\left\|b\left(e_{s}, F\right)\right\|\|G\| \\
& =\| b\left(e_{s}, \sum_{r}\left(J e_{r}\right) \otimes f_{r}\|\| G\left\|=\sum_{r} \sigma\left(e_{s}, J e_{r}\right) f_{r}\right\|\|G\|\right. \\
& =\left\|\sum_{r}<J e_{s}, J e_{r}>f_{r}\right\|\|G\|=\left\|f_{s}\right\|\|G\| .
\end{aligned}
$$

(The continuity of $b$ was used.) Therefore,

$$
\sum_{s}\left\|B_{s}\right\|^{2} \leq\|G\|^{2} \sum_{s}\left\|f_{s}\right\|^{2}=\|G\|^{2}\|F\|^{2}<\infty
$$

and $\sum_{s} B_{s}$ is a well-defined vector in $H^{2}$. But for this vector, one has

$$
\begin{aligned}
b\left(h, \sum_{s} B_{s}\right) & =\sum_{s} b\left(h, B_{s}\right)=\sum_{s} \sigma\left(h, J e_{s}\right) b\left(b\left(e_{s}, F\right), G\right) \\
& =b\left(b\left(\sum_{s}\left\langle J h, J e_{s}\right\rangle e_{s}, F\right), G\right)=b(b(h, F), G) .
\end{aligned}
$$

2. Let $B_{1}$ and $B_{2}$ be two vectors in $H^{2}$ such that $b\left(h, B_{1}\right)=$ $b\left(h, B_{2}\right)=b(b(h, F), G) \forall h \in H$ for given $F, G \in H^{2}$. Then $b\left(h, B_{1}-B_{2}\right)=0$ and $\left\langle b\left(h, B_{1}-B_{2}\right), g\right\rangle=\sigma\left(h \otimes g, B_{1}-B_{2}\right)=0$ for all $g, h \in H$. Since, however, $\sigma(\cdot, \cdot)$ is linear and continuous in the first entry, one has therefore $\sigma\left(K, B_{1}-B_{2}\right)=0$ for all $K \in H^{2}$. But by the nondegeneracy of $\sigma$, it follows that $B_{1}=B_{2}$. 
3. From step (1) one obtains the bound $\|B(F, G)\| \leq\|F\|\|G\|$ and thus the continuity of $B$.

Note that from the above arguments, if $\left\{e_{s}\right\}$ is an orthonormal basis in $H$ and $f_{1}, f_{2}, g_{1}, g_{2} \in H$, then

$$
\begin{aligned}
B\left(f_{1} \otimes f_{2}, g_{1} \otimes g_{2}\right) & =\sum_{s}\left(J e_{s}\right) \otimes b\left(b\left(e_{s}, f_{1} \otimes f_{2}\right), g_{1} \otimes g_{2}\right) \\
& =\sum_{s} \sigma\left(e_{s}, f_{1}\right) \sigma\left(f_{2}, g_{1}\right)\left(J e_{s}\right) \otimes g_{2} \\
& =\sigma\left(f_{2}, g_{1}\right) \sum_{s}\left\langle J e_{s}, f_{1}\right\rangle\left(J e_{s}\right) \otimes g_{2}=\sigma\left(f_{2}, g_{1}\right) f_{1} \otimes g_{2},
\end{aligned}
$$

in analogy to $(3.2 .6)$.

We have introduced the maps $b$ and $B$, because they appear in the computation of the commutators of transformed fields, as we see in the next theorem.

Proposition 3.2.6. For every $f \in H, F, G \in P_{+} H^{2}$, and $\varphi \in \mathscr{F}_{0}$ one has

$$
\begin{aligned}
& {[\Phi(f), \Psi(G)] \varphi=2 i \Phi(b(f, G)) \varphi} \\
& {[\Psi(F), \Psi(G)] \varphi=4 i \Psi\left(P_{+} B(F, G)\right) \varphi+2 i \sigma(F, G) \varphi}
\end{aligned}
$$

It therefore follows that for any $\Lambda \in \mathscr{L}$, one has

$$
\begin{aligned}
{\left[\Phi_{\Lambda}(f), \Phi_{\Lambda}(g)\right] \varphi=} & i \sigma(f, g) \varphi+2 i \Phi(b(f, \Lambda g)-b(g, \Lambda f)) \varphi \\
& +4 i \Psi\left(P_{+} B(\Lambda f, \Lambda g)\right) \varphi+2 i \sigma(\Lambda f, \Lambda g) \varphi
\end{aligned}
$$

Proof. To begin, let $G$ be of the form $G=P_{+}\left(g_{1} \otimes g_{2}\right)$. Then one computes

$$
\begin{aligned}
{\left[\Phi(f), \Psi\left(P_{+}\left(g_{1} \otimes g_{2}\right)\right)\right] \varphi } & =\left[\Phi(f), \Psi\left(g_{1} \otimes g_{2}\right)\right] \varphi=\left[\Phi(f),: \Phi\left(g_{1}\right) \Phi\left(g_{2}\right):\right] \varphi \\
& =\left[\Phi(f), \Phi\left(g_{1}\right) \Phi\left(g_{2}\right)\right] \varphi \\
& =\left[\Phi(f), \Phi\left(g_{1}\right)\right] \Phi\left(g_{2}\right) \varphi+\Phi\left(g_{1}\right)\left[\Phi(f), \Phi\left(g_{2}\right)\right] \varphi \\
& =i \sigma\left(f, g_{1}\right) \Phi\left(g_{2}\right) \varphi+i \sigma\left(f, g_{2}\right) \Phi\left(g_{1}\right) \varphi \\
& =i \Phi\left(b\left(f, g_{1} \otimes g_{2}\right)+b\left(f, g_{2} \otimes g_{1}\right)\right) \varphi \\
& =2 i \Phi\left(b\left(f, P_{+}\left(g_{1} \otimes g_{2}\right)\right)\right) \varphi
\end{aligned}
$$

This equation holds as well for the dense linear span of such simple tensor products and hence for all $G \in P_{+} H^{2}$, as soon as it is seen that both sides of the equation are continuous in $G$. First, the map $G \mapsto 2 i \Phi(b(f, G)) \varphi$ is continuous, since both maps $G \mapsto b(f, G)$ and $h \mapsto \Phi(h) \varphi$ are continuous. The map $G \mapsto[\Phi(f)$, $\Psi(G)] \varphi$ is also bounded: 


$$
\begin{aligned}
\|[\Phi(f), \Psi(G)] \varphi\| & \leq\|\Phi(f) \Psi(G) \varphi\|+\|\Psi(G) \Phi(f) \varphi\| \\
& \leq \sqrt{2} \sqrt{n_{\varphi}+3}\|f\|\|\Psi(G) \varphi\|+2 \sqrt{2} \sqrt{\left(n_{\varphi}+2\right)\left(n_{\varphi}+3\right)}\|G\|\|\Phi(f) \varphi\| \\
& \leq 8 \sqrt{\left(n_{\varphi}+1\right)\left(n_{\varphi}+2\right)\left(n_{\varphi}+3\right)}\|f\|\|G\|\|\varphi\|
\end{aligned}
$$

(see $(3.1 .1))$.

With $\left\{e_{s}\right\}$ an orthonormal basis in $H, F=\sum_{r, s} \alpha_{r s} e_{r} \otimes e_{s}, G=\sum_{p, q} \gamma_{p q} e_{p} \otimes e_{q}$, $\alpha_{r s}=\alpha_{s r}, \gamma_{p q}=\gamma_{q p} \in \mathbf{R}$ and $\sigma_{r s} \equiv \sigma\left(e_{r}, e_{s}\right)$, one has

$$
\begin{aligned}
{[\Psi(F), \Psi(G)] \varphi=} & \sum_{p, q} \gamma_{p q}\left[\Psi(F), \Phi\left(e_{p}\right) \Phi\left(e_{q}\right)\right] \varphi \\
= & \sum_{p, q} \gamma_{p q}\left\{\Phi\left(e_{p}\right)\left[\Psi(F), \Phi\left(e_{q}\right)\right]+\left[\Psi(F), \Phi\left(e_{p}\right)\right] \Phi\left(e_{q}\right)\right\} \varphi \\
= & -2 i \sum_{p, q} \gamma_{p q}\left\{\Phi\left(e_{p}\right) \Phi\left(b\left(e_{q}, F\right)\right)+\Phi\left(b\left(e_{p}, F\right)\right) \Phi\left(e_{q}\right)\right\} \varphi \\
= & -2 i \sum_{p, q} \gamma_{p q}\left\{: \Phi\left(e_{p}\right) \Phi\left(b\left(e_{q}, F\right)\right):+\frac{1}{2}\left\langle e_{p}, b\left(e_{q}, F\right)\right\rangle\right. \\
& +\frac{i}{2} \sigma\left(e_{p}, b\left(e_{q}, F\right)\right)+: \Phi\left(b\left(e_{p}, F\right)\right) \Phi\left(e_{q}\right): \\
& \left.+\frac{1}{2}\left\langle b\left(e_{p}, F\right), e_{q}\right\rangle+\frac{i}{2} \sigma\left(b\left(e_{p}, F\right), e_{q}\right)\right\} \varphi \\
= & -2 i \sum_{p, q} \gamma_{p q}\left\{\Psi\left(\sum_{r, s} \alpha_{r s}\left(\sigma_{q} e_{p} \otimes_{e_{s}}+\sigma_{p r} e_{s} \otimes_{e_{q}}\right)\right)\right. \\
& +\frac{1}{2} \sigma\left(e_{q} \otimes_{e_{p}}+e_{p} \otimes e_{q}, F\right)+\frac{i}{2}\left(\sigma\left(e_{p}, b\left(e_{q}, F\right)\right)\right. \\
& \left.\left.-\sigma\left(e_{q}, b\left(e_{p}, F\right)\right)\right)\right\} \varphi .
\end{aligned}
$$

The last term vanishes, because it is antisymmetric and $\gamma_{p q}$ is symmetric in $p$ and $q$, so with

$$
\begin{aligned}
P_{+} B(F, G) & =\sum_{p, q} \gamma_{p q} \sum_{r, s} \alpha_{r s} \sigma_{s p} P_{+}\left(e_{r} \otimes e_{q}\right) \\
& =\frac{1}{2}\left(\sum_{p, q} \gamma_{p q} \sum_{r, s} \alpha_{r s} \sigma_{r p} e_{s} \otimes e_{q}+\sum_{p, q} \gamma_{p q} \sum_{r, s} \alpha_{r s} \sigma_{r q} e_{p} \otimes e_{s}\right)
\end{aligned}
$$

and

$$
\sigma(F, G)=\sum_{p, q} \gamma_{p q} \sigma\left(F, e_{p} \otimes e_{q}\right)=\sum_{p, q} \gamma_{p q} \sigma\left(F, e_{q} \otimes e_{p}\right)
$$

one obtains the right side of equation (3.2.8).

Remark. Note that the first two equations in the Proposition are valid only for symmetric $F, G \in H^{2}$.

A first result characterizing canonical quadratic transformations can now 
be given.

Proposition 3.2.7. Let $\Lambda \in \mathscr{L}$. The transformation $\Phi(f) \mapsto \Phi_{\Lambda}(f), f \in D(\Lambda)$, is canonical if and only if, for all $f, g \in D(\Lambda)$, one has

$$
b(f, \Lambda g)=b(g, \Lambda f)
$$

and

$$
P_{+} B(\Lambda f, \Lambda g)=0
$$

Proof. 1. $\quad(\Leftrightarrow)$ If equations (3.2.11) and (3.2.12) are satisfied, then by (3.2.9) the transformation is canonical, whenever $\sigma(\Lambda f, \Lambda g)=0$ holds. This, then, will be proven. Choose an orthonormal basis $\left\{e_{s}\right\}$ in $H$ and write

$$
\Lambda f=\sum_{r, s} \alpha_{r s} e_{r} \otimes e_{s}=\sum_{r} e_{r} \otimes f_{r}=\sum_{r} f_{r} \otimes e_{r}
$$

$\left(\alpha_{r s}=\alpha_{s r} \in \mathbf{R}, f_{r} \equiv \sum_{s} \alpha_{r s} e_{s}\right)$ and correspondingly $\Lambda g=\sum_{s} g_{s} \otimes e_{s}$. Then one sees that

$$
0=P_{+} B(\Lambda f, \Lambda g)=P_{+} \sum_{r, s} B\left(e_{r} \otimes f_{r}, g_{s} \otimes e_{s}\right)=\sum_{r, s} \sigma\left(f_{r}, g_{s}\right) P_{+}\left(e_{r} \otimes e_{s}\right)
$$

in particular, $\sigma\left(f_{r}, g_{r}\right)=0$ for every $r$, and thus

$$
\sigma(\Lambda f, \Lambda g)=\sum_{r, s} \sigma\left(f_{r} \otimes_{e_{r},} g_{s} \otimes_{e_{s}}\right)=\sum_{r, s} \sigma\left(f_{r}, g_{s}\right)\left\langle e_{r}, e_{s}\right\rangle=\sum_{r} \sigma\left(f_{r}, g_{r}\right)=0
$$

2. $(\Rightarrow)$ On the other hand, if $\Phi(f) \mapsto \Phi_{\Lambda}(f)$ is canonical, then by equation (3.2.9) one has

$$
2 \Psi(K) \varphi+\Phi(k) \varphi+\sigma(\Lambda f, \Lambda g) \varphi=0 \quad \forall \varphi \in \mathscr{F}_{0}
$$

where the quantity $P_{+} B(\Lambda f, \Lambda g)$ has been designated by $K$ and $b(f, \Lambda g)-b(g$, $\Lambda f$ ) by $k$. One must therefore show that $K=0$ and $k=0$. With this goal in mind, by (3.2.13) one has

$$
\begin{aligned}
0 & =\left[\Phi\left(h_{1}\right),\left[\Phi\left(h_{2}\right), 2 \Psi(K)+\Phi(k)+\sigma(\Lambda f, \Lambda g) 1\right]\right] \varphi \\
& =2\left[\Phi\left(h_{1}\right),\left[\Phi\left(h_{2}\right), \Psi(K)\right]\right] \varphi=4 i\left[\Phi\left(h_{1}\right), \Phi\left(b\left(h_{2}, K\right)\right)\right] \varphi
\end{aligned}
$$

for all $h_{1}, h_{2} \in H, \varphi \in \mathscr{F}_{0}$, where in the last step equation (3.2.7) was used. This leads to 
$0=-4 \sigma\left(h_{1}, b\left(h_{2}, K\right)\right) \varphi=-4 \sigma\left(h_{2} \otimes\left(J h_{1}\right), K\right) \varphi=-4\left\langle\left(J h_{2}\right) \otimes\left(J h_{1}\right), K\right\rangle \varphi$,

so that $K=0$. Equation (3.2.13) then entails that $\Phi(k) \varphi+\sigma(\Lambda f, \Lambda g) \varphi=0$, from which it follows that $k=0$.

Remark 3.2.8. We next note that if $F=\sum_{r} f_{r} \otimes_{e_{r}}=\sum_{r_{r}} \otimes f_{r} \in P_{+} H^{2}$ and $G=$ $\sum_{s} g_{s} \otimes e_{s}=\sum_{s} e_{s} \otimes g_{s} \in P_{+} H^{2}$, then

$$
\begin{aligned}
2 P_{+} B(F, G) & =\sum_{r, s} \sigma\left(f_{r}, g_{s}\right)\left(e_{r} \otimes e_{s}+e_{s} \otimes e_{r}\right) \\
& =\sum_{r, s}\left(\sigma\left(f_{r}, g_{s}\right) e_{r} \otimes e_{s}-\sigma\left(g_{s}, f_{r}\right) e_{s} \otimes_{e_{r}}\right)=B(F, G)-B(G, F)
\end{aligned}
$$

thus one has $P_{+} B(\Lambda f, \Lambda g)=0$ if and only if $B(\Lambda f, \Lambda g)=B(\Lambda g, \Lambda f)$.

A variant of Proposition 3.2.7 will now be presented, which demonstrates that if the quadratic transformation is canonical, then not only the symmetric part of $B(\Lambda f, \Lambda g)$ must be zero, but, in fact, also the antisymmetric part vanishes.

Proposition 3.2.9. Let $\Lambda \in \mathscr{L}$. The transformation $\Phi(f) \mapsto \Phi_{\Lambda}(f), f \in D(\Lambda)$, is canonical if and only if, for all $f, g \in D(\Lambda)$, one has

$$
b(f, \Lambda g)=b(g, \Lambda f)
$$

and

$$
B(\Lambda f, \Lambda g)=0
$$

Proof. Given the preceding proposition, one may assume that (3.2.14) and (3.2.12) hold. Using the equality

$$
\langle B(F, G),(J h) \otimes(J k)\rangle=\sigma(b(h, F), b(k, G))
$$

(for general $F, G \in P_{+} H^{2}, h, k \in H$ ) and Remark 3.2.8, one finds for $f, g, h, k \in$ $D(\Lambda)$ :

$$
\begin{aligned}
\langle B(\Lambda f, \Lambda g),(J h) \otimes(J k)\rangle=\sigma(b(h, \Lambda f), b(k, \Lambda g))=-\sigma(b(k, \Lambda g), b(h, \Lambda f)) \\
=-\langle B(\Lambda g, \Lambda f),(J k) \otimes(J h)\rangle=-\langle B(\Lambda f, \Lambda g),(J k) \otimes(J h)\rangle \\
=-\sigma(b(k, \Lambda f), b(h, \Lambda g))=-\sigma(b(f, \Lambda k), b(g, \Lambda h)) \\
=-\langle B(\Lambda k, \Lambda h),(J f) \otimes(J g)\rangle=-\langle B(\Lambda h, \Lambda k),(J f) \otimes(J g)\rangle \\
=-\sigma(b(f, \Lambda h), b(g, \Lambda k))=-\sigma(b(h, \Lambda f), b(k, \Lambda g))
\end{aligned}
$$




$$
=-\langle B(\Lambda f, \Lambda g),(J h) \otimes(J k)\rangle .
$$

Since $D(\Lambda)$ is dense in $H$, the set of vectors of the form $(J h) \otimes(J k)$ is total in $H^{2}$; hence it follows that $B(\Lambda f, \Lambda g)=0$.

We next reformulate condition (3.2.15) in a more symplectic geometrical context.

Lemma 3.2.10. Let $\Lambda \in \mathscr{L}$. The condition (3.2.15) holds exactly when there exists a closed isotropic subspace $M \subset H$ (i.e. $\sigma(m, n)=0, \forall m, n \in M)$ such that $R(\Lambda) \subset M \otimes M$.

Proof. Since it has been shown that the range of $\Lambda$ consists of symmetric vectors, the relation $R(\Lambda) \subset M \otimes M$ may be replaced by $R(\Lambda) \subset P_{+}(M \otimes M)$.

1. $(\Leftarrow)$ Let $f, g \in D(\Lambda)$ and $\left\{n_{i}\right\}$ be an orthonormal basis in $M$, which by hypothesis must satisfy $\sigma\left(n_{i}, n_{j}\right)=0$. Since by assumption the vectors $\Lambda f$ and $\Lambda g$ are elements of $M \otimes M$, one may write $\Lambda f=\sum_{i} f_{i} \otimes n_{i}$ and $\Lambda g=\sum_{j} n_{j} \otimes g_{j}$, with $f_{i}$, $g_{j} \in M$. Employing the continuity of $B$, one obtains

$$
B(\Lambda f, \Lambda g)=\sum_{i} \sum_{j} B\left(f_{i} \otimes n_{i}, n_{j} \otimes g_{j}\right)=\sum_{i} \sum_{j} \sigma\left(n_{i}, n_{j}\right) f_{i} \otimes g_{j}=0 .
$$

2. $(\Rightarrow)$ In this direction the isotropic subspace will be explicitly constructed. If $\left\{e_{s}\right\}$ is an orthonormal basis in $H$ and one writes $F \in \mathrm{R}(\Lambda)$ in the form $F=\sum_{s} f_{s} \otimes e_{s}$, then one obtains the vectors $f_{1}, f_{2}, f_{3}, \ldots \in H$. This leads to a map from an element $F \in \mathrm{R}(\Lambda)$ to a set $\left\{f_{s}\right\}$ of vectors. The range of this map, as $F$ runs through $\mathrm{R}(\Lambda)$, will be called $M_{0}$. The closure of the linear span of $M_{0}$ will be called $M$. This will be shown to be the desired subspace.

Let $f$ and $g$ be chosen from $D(\Lambda)$; then one has $\Lambda f=\sum_{r} f_{r} \otimes_{e_{r}}=\sum_{r_{r}} \otimes f_{r}$ (since $\Lambda f$ is symmetric), $\Lambda g=\sum_{s} g_{s} \otimes e_{s}$, and $f_{r}, g_{s} \in M_{0}$. If

$$
0=B(\Lambda f, \Lambda g)=\sum_{r} \sum_{s} \sigma\left(f_{r}, g_{s}\right) e_{r} \otimes e_{s}
$$

then $\sigma\left(f_{r}, g_{s}\right)=0$ and $\sigma(f, g)=0$ hold for all $f, g \in M_{0}$. Since the set $M_{0}$ is total in $M$, it therefore follows that $\sigma(m, n)=0$ for all $m, n \in M$.

On the other hand, if $F \in \mathrm{R}(\Lambda)$, then also $F \in M \otimes H$, and therefore one may write $F=\sum_{i} n_{i} \otimes \widehat{f_{i}}$, with an orthonormal basis $\left\{n_{i}\right\}$ in $M$ and $\widehat{f_{i}} \in H$. For an arbitrary element $n_{j}$ of this basis and an $h \in H$, one then has

$$
\left\langle F, n_{j} \otimes h\right\rangle=\sum_{i}\left\langle n_{i}, n_{j}\right\rangle\left\langle\widehat{f}_{i}, h\right\rangle=\left\langle\widehat{f}_{j}, h\right\rangle
$$


But $F$ is symmetric, so one also has

$$
\left\langle F, n_{j} \otimes h\right\rangle=\left\langle F, h \otimes n_{j}\right\rangle=\sum_{i}\left\langle n_{i}, h\right\rangle\left\langle\widehat{f}_{i}, n_{j}\right\rangle
$$

Choosing $h$ from the orthogonal space $M^{\perp}$ to $M$, one concludes $\left\langle\widehat{f}_{j}, h\right\rangle=0, \forall h \in$ $M^{\perp}$, in other words one has $\widehat{f}_{j} \in M^{\perp \perp}=\bar{M}=M$. But since then all $\widehat{f}_{i}$ in the sum $F=\sum_{i} n_{i} \otimes \widehat{f}_{i}$ belong to $M$, one clearly has $F \in M \otimes M$, which completes the proof.

We summarize the main results of this section up to this point in the following theorem.

Theorem 3.2.11. The transformation $\Phi(f) \mapsto \Phi_{\Lambda}(f), f \in D(\Lambda)$ with $\Lambda \in \mathscr{L}$ is canonical (i.e., $\left.\Lambda \in \mathscr{L}_{C C R}\right)$ if and only if $b(f, \Lambda g)=b(g, \Lambda f)$ for all $f, g \in D(\Lambda)$ and $R(\Lambda) \subset M \otimes M$ for some closed, isotropic subspace $M$ of $H$.

\section{§3.3. The Standard Form of Canonical Quadratic Transformations}

In this section we shall prove that if $\Lambda \in \mathscr{L}_{C C R}$, then there exists a suitable basis in $H$ in terms of which the map $\Lambda$ has a particularly convenient form. In fact, we shall be able to explicitly characterize the elements of $\mathscr{L}_{C C R}$ in this manner. This technical point is crucial in the arguments of the following sections.

Proposition 3.3.1. For any $\Lambda \in \mathscr{L}_{C C R}$ there exists a symplectic orthonormal basis $\left\{e_{k}, e_{k^{\prime}}\right\}=\left\{e_{K}: K=1,1^{\prime}, 2,2^{\prime}, \ldots\right\}$ in $H$ and real numbers $\lambda_{i j k}, i, j, k=1,2,3$, ..., which are completely symmetric in the three indices, and satisfy

$$
\sum_{i, j} \lambda_{i j k}^{2}<\infty
$$

for every $k$, such that the operator $\Lambda$ may be expressed as:

$$
\Lambda f=\sum_{i, j} \sum_{k} \lambda_{i j k} \sigma\left(e_{k}, f\right) e_{i} \otimes e_{j}
$$

for all $f \in D(\Lambda)$.

Proof. 1. From Theorem 3.2.11 it is known that there exists a closed isotropic subspace $M$ of $H$ with $\mathrm{R}(\Lambda) \subseteq M \otimes M$. Let $\left\{m_{i}\right\}$ be an orthonormal basis in $M$ and consider the corresponding vectors $\tilde{m}_{i}$ in $\mathscr{H}$. Since one has $\left\langle\widetilde{m}_{i}, \tilde{m}_{j}\right\rangle_{\mathscr{H}}=$ 
$\left\langle m_{i}, m_{j}\right\rangle+\mathrm{i} \sigma\left(m_{i}, m_{j}\right)=\left\langle m_{i}, m_{j}\right\rangle=\delta_{i j}$, these vectors form an orthonormal system, which can be completed in the case that it is not already complete in $\mathscr{H}$. From the discussion in Section 2, the resulting orthonormal basis $\left\{\tilde{n}_{k}\right\}$ in $\mathscr{H}$ can be transformed into a symplectic orthonormal basis $\left\{n_{k}, J n_{k}\right\}=\left\{n_{k}, n_{k^{\prime}}\right\}=\left\{n_{K}\right\}$ in $H$. In the subset $\left\{n_{k}\right\}$ of this system are contained the original vectors $m_{i}$. Calling $N \subset H$ the closed subspace spanned by $\left\{n_{k}\right\}$, one has by construction: $M \subset N$, $\mathrm{R}(\Lambda) \subset N \otimes N$ and $\sigma(m, n)=0$, for any $m, n \in N$. One may therefore replace $M$ by $N$, which has the hypothesized properties of $M$ and which, moreover, has the advantage of being a maximal isotropic subspace of $H$. Note that one has $J N=N^{\perp}$ and $N \oplus(J N)=H$.

Next let $f \in D(\Lambda), g \in H$ and $n \in N \cap D(\Lambda)$. Since $\Lambda f \in N \otimes N$, it may be written as $\Lambda f=\sum_{i} f_{i} \otimes g_{i}$, with $f_{i}, g_{i} \in N$. Thus one has $\sigma(n \otimes g, \Lambda f)=\sum_{i} \sigma\left(n, f_{i}\right)<g$, $\left.g_{i}\right\rangle=0$. Using (3.2.14) one finds then that

$$
\sigma(f \otimes g, \Lambda n)=\langle b(f, \Lambda n), g\rangle=\langle b(n, \Lambda f), g\rangle=\sigma(n \otimes g, \Lambda f)=0
$$

which entails $\Lambda n=0$. One may therefore extend $\Lambda$ to an operator $\hat{\Lambda}$ by defining:

$$
\begin{aligned}
D(\widehat{\Lambda}) & \equiv D(\Lambda)+N=\{f \in H \mid f=l+n, l \in D(\Lambda), n \in N\}, \\
\widehat{\Lambda} f & =\widehat{\Lambda}(l+n) \equiv \Lambda l,
\end{aligned}
$$

where $f=l+n \in D(\widehat{\Lambda}), l \in D(\Lambda)$, and $n \in N$. Since the decomposition $f=l+n$ need not be unique, it must be checked that the definition (3.3.2) makes sense. Therefore, consider two decompositions $f=l_{1}+n_{1}, f=l_{2}+n_{2}$ of $f \in D(\widehat{\Lambda})\left(l_{1}, l_{2} \in\right.$ $\left.D(\Lambda), n_{1}, n_{2} \in N\right)$. Since $n_{1}-n_{2}=l_{2}-l_{1}$, one has $n_{1}-n_{2} \in N \cap D(\Lambda)$; hence $\Lambda\left(n_{1}-\right.$ $\left.n_{2}\right)=0$, as has just been shown above. Thus one has $\Lambda l_{2}=\Lambda\left(n_{1}-n_{2}+l_{1}\right)=\Lambda l_{1}$ and $\widehat{\Lambda}\left(l_{2}+n_{2}\right)=\Lambda l_{2}=\Lambda l_{1}=\widehat{\Lambda}\left(l_{1}+n_{1}\right)$. It is straightforward to verify that $D(\widehat{\Lambda})$ is a linear subspace of $H$, and that $\hat{\Lambda}$ is linear. Moreover, one also has $\mathrm{R}(\hat{\Lambda})=$ $\mathrm{R}(\Lambda)$ and $\hat{\Lambda} \in \mathscr{L}$.

If $\Lambda \in \mathscr{L}_{C C R}$, then also for its extension one has $\widehat{\Lambda} \in \mathscr{L}_{C C R}$, as can be seen: Since the condition $\mathrm{R}(\widehat{\Lambda})=\mathrm{R}(\Lambda) \subset N \otimes N$ has already been verified, one needs only check (3.2.14). But for arbitrary $f_{1}=l_{1}+n_{1}, f_{2}=l_{2}+n_{2} \in D(\widehat{\Lambda})$, one computes

$$
b\left(f_{1}, \widehat{\Lambda} f_{2}\right)=b\left(l_{1}+n_{1}, \Lambda l_{2}\right)=b\left(l_{1}, \Lambda l_{2}\right)=b\left(l_{2}, \Lambda l_{1}\right)=b\left(f_{2}, \widehat{\Lambda} f_{1}\right)
$$

2. Since $\widehat{\Lambda} f=\Lambda f$, for any $f \in D(\Lambda)$, it will suffice to show that the theorem holds for the extended operator. This will be technically easier. Let $P$ be the orthogonal projection onto the subspace $N$, so that $P^{\perp}=I-P$ is the orthogonal projection onto $N^{\perp}=J N$. The set $P^{\perp} D(\widehat{\Lambda})$ is dense in $N^{\perp}$ and is a separable pre- 
Hilbert space; therefore it contains an orthonormal basis $\left\{e_{k^{\prime}}\right\}$, which is also an orthonormal basis for $\overline{P^{\perp} D(\widehat{\Lambda})}=N^{\perp}$. Hence $\left\{-J e_{k^{\prime}}\right\}$ is an orthonormal basis in $-J N^{\perp}=-J J N=N$, and if one sets $-J e_{k^{\prime}} \equiv e_{k}$, then $J e_{k}=e_{k^{\prime}}$ and $\left\{e_{k}, e_{k^{\prime}}\right\}=\left\{e_{K}\right\}$ is a symplectic orthonormal basis in $H$ contained in $D(\widehat{\Lambda})$ : the containment $\left\{e_{k}\right\} \subset N$ $\subseteq D(\widehat{\Lambda})$ is clear. Now, if $f$ is an arbitrary element in $D(\widehat{\Lambda})$, then $P f \in N \subset D(\widehat{\Lambda})$ and $P^{\perp} f=f-P f \in D(\widehat{\Lambda})$. In other words, $P^{\perp} D(\widehat{\Lambda}) \subset D(\widehat{\Lambda})$. However, as $\left\{e_{k^{\prime}}\right\}$ is by construction a subset of $P^{\perp} D(\widehat{\Lambda})$, one also has $\left\{e_{k^{\prime}}\right\} \subset D(\widehat{\Lambda})$.

3. It shall be seen that the constructed basis $\left\{e_{K}\right\}$ and the numbers defined by $\lambda_{i j k} \equiv\left\langle e_{i} \otimes e_{j}, \widehat{\Lambda} J e_{k}\right\rangle$ satisfy the conclusions of the proposition. First, since $\widehat{\Lambda} e_{k} \in P_{+} H^{2}$, it follows that $\lambda_{i j k}=\lambda_{j i k}$. Moreover, one has

$$
\lambda_{i j k}=-\sigma\left(\left(J e_{i}\right) \otimes e_{j}, \widehat{\Lambda} J e_{k}\right)=-\left\langle b\left(J e_{i}, \widehat{\Lambda} J e_{k}\right), e_{j}\right\rangle=-\left\langle b\left(J e_{k}, \hat{\Lambda} J e_{i}\right), e_{j}\right\rangle=\lambda_{k j i}
$$

This implies that $\lambda_{i j k}$ is invariant under exchange of the first with the second, as well as the first with the third index, so it is completely symmetric in the three indices.

Next it is noted that

$$
\begin{aligned}
\infty>\left\|\hat{\Lambda} J e_{k}\right\|^{2} & =\| \sum_{I, J}\left\langle e_{I} \otimes_{e_{J}}, \widehat{\Lambda} J e_{k}>e_{I} \otimes e_{J} \|^{2}\right. \\
& =\sum_{I, J}<e_{I} \otimes e_{J}, \widehat{\Lambda} J e_{k}>^{2} \geq \sum_{i, j}<e_{i} \otimes e_{j}, \widehat{\Lambda} J e_{k}>^{2}=\sum_{i, j} \lambda_{i j k}^{2},
\end{aligned}
$$

for every $k$, since $J e_{k} \in D(\widehat{\Lambda})$. Further, for $f \in D(\widehat{\Lambda})$ one has

$$
\widehat{\Lambda} f=\sum_{I, J}\left\langle e_{I} \otimes e_{J}, \widehat{\Lambda} f\right\rangle e_{I} \otimes e_{J}=\sum_{I, J}\left\langle e_{I} \otimes e_{J}, \widehat{\Lambda} \sum_{K}\left\langle J e_{K}, f\right\rangle J e_{K}\right\rangle e_{I} \otimes e_{J}
$$

Employing once again $(3.2 .14)$, one sees that for any $e_{I}, e_{J}$ the linear map

$$
f \mapsto\left\langle e_{I} \otimes e_{J}, \widehat{\Lambda} f\right\rangle=-\left\langle b\left(J e_{I}, \widehat{\Lambda} f\right), e_{J}\right\rangle=-\left\langle b\left(f, \widehat{\Lambda} e_{I}\right), e_{J}\right\rangle
$$

is bounded, since $\|b(f, F)\| \leq\|f\|\|F\|$. Thus, one may pull the infinite sum through the scalar product above to obtain

$$
\widehat{\Lambda} f=\sum_{I, J} \sum_{K}\left\langle J e_{K}, f\right\rangle\left\langle e_{I} \otimes e_{J}, \widehat{\Lambda} J e_{K}\right\rangle e_{I} \otimes_{e_{J}}
$$

Since on the one hand, $\widehat{\Lambda} J e_{K} \in N \otimes N$, and on the other hand, $\widehat{\Lambda} J N^{\perp}=\widehat{\Lambda} N=\{0\}$, it follows that the only scalar products satisfying $\left\langle e_{I} \otimes_{e_{J}}, \hat{\Lambda} J e_{K}\right\rangle \neq 0$ are those for which $e_{I}, e_{J}, e_{K} \in N$, in other words, the vectors must come from $\left\{e_{k}\right\}$. Thus, one 
has finally

$$
\widehat{\Lambda} f=\sum_{i, j} \sum_{k}\left\langle J e_{k}, f\right\rangle\left\langle e_{i} \otimes e_{j}, \widehat{\Lambda} J e_{k}\right\rangle e_{i} \otimes_{e_{j}}=\sum_{i, j} \sum_{k} \sigma\left(e_{k}, f\right) \lambda_{i j k} e_{i} \otimes_{e_{j}}
$$

The next step in our program to explicitly characterize the elements of $\mathscr{L}_{C C R}$ is the following proposition, which is in some sense the converse of the preceding result.

Proposition 3.3.2. Let $\left\{e_{k}\right\}$ be an orthonormal system in $H$ with $\sigma\left(e_{k}, e_{l}\right)=0$ for all $k, l$, and let $\lambda_{i j k}$ be real numbers which are completely symmetric in the three indices satisfying $\sum_{i, j} \lambda_{i j k}^{2}<\infty$ for every $k$. Then the operator $\Lambda: H \mapsto H^{2}$ defined by

$$
\begin{aligned}
D(\Lambda) & \equiv\left\{f \in H \mid \sum_{i, j}\left(\sum_{k} \lambda_{i j k} \sigma\left(e_{k}, f\right)\right)^{2}<\infty\right\}, \\
\Lambda f & \equiv \sum_{i, j} \sum_{k} \lambda_{i j k} \sigma\left(e_{k}, f\right) e_{i} \otimes e_{j},
\end{aligned}
$$

for $f \in D(\Lambda)$ is contained in $\mathscr{L}_{C C R}$, i.e. the quadratic transformation $\Phi(f) \mapsto \Phi_{\Lambda}(f)$, $f \in D(\Lambda)$, is canonical.

Proof. 1. It shall be assumed that $\left\{e_{k}\right\}$ is an infinite orthonormal system, and that the indices $i, j, k$ run from 1 to infinity, since the finite case is relatively trivial. The first step is to show that for every $i, j$ and $f \in H$ the series $\sum_{k} \lambda_{i j k} \sigma\left(e_{k}, f\right)$ is absolutely convergent. Since $\left\{J e_{k}\right\}$ is also an orthonormal system in $H$, Bessel's inequality yields $\|f\|^{2} \geq \sum_{k}\left\langle J e_{k}, f\right\rangle^{2}=\sum_{k} \sigma\left(e_{k}, f\right)^{2}$, for $f \in H$. Thus the sequence $\varphi \equiv\left\{\left|\sigma\left(e_{1}, f\right)\right|,\left|\sigma\left(e_{2}, f\right)\right|,\left|\sigma\left(e_{3}, f\right)\right|, \ldots\right\}$ belongs to $\ell_{2}(\mathbf{R})$ and $\|\varphi\|_{\ell_{2}}^{2} \leq\|f\|^{2}$. In addition, the sequence $\lambda_{i j} \equiv\left\{\left|\lambda_{i j 1}\right|,\left|\lambda_{i j 2}\right|,\left|\lambda_{i j 3}\right|, \ldots\right\} \in \ell_{2}(\mathbf{R})$, since $\sum_{i, j} \lambda_{k i j}^{2}<\infty$ entails $\sum_{j} \lambda_{k i j}^{2}<\infty$. In the Hilbert space $\ell_{2}(\mathbf{R})$ the Cauchy-Schwarz inequality implies:

$$
\left\langle\lambda_{i j}, \varphi\right\rangle_{\ell_{2}}=\sum_{k}\left|\lambda_{i j k}\right|\left|\sigma\left(e_{k}, f\right)\right| \leq\left\|\lambda_{i j}\right\|_{\ell_{2}}\|\varphi\|_{\ell_{2}}<\infty
$$

so that $\sum_{k} \lambda_{i j k} \sigma\left(e_{k}, f\right)$ is absolutely convergent.

2. The next step is to show that $\Lambda \in \mathscr{L}$. The linearity of $\Lambda$ is clear, once it is seen that $f, g \in D(\Lambda)$ implies that $f+g \in D(\Lambda)$. But, in fact,

$$
\begin{aligned}
\sum_{i, j}\left(\sum_{k} \lambda_{i j k} \sigma\left(e_{k}, f+g\right)\right)^{2} & =\sum_{i, j}\left(\sum_{k} \lambda_{i j k} \sigma\left(e_{k}, f\right)+\sum_{k} \lambda_{i j k} \sigma\left(e_{k}, g\right)\right)^{2} \\
& \leq 2 \sum_{i, j}\left\{\left(\sum_{k} \lambda_{i j k} \sigma\left(e_{k}, f\right)\right)^{2}+\left(\sum_{k} \lambda_{i j k} \sigma\left(e_{k}, g\right)\right)^{2}\right\} \\
& =2 \sum_{i, j}\left(\sum_{k} \lambda_{i j k} \sigma\left(e_{k}, f\right)\right)^{2}+2 \sum_{i, j}\left(\sum_{k} \lambda_{i j k} \sigma\left(e_{k}, g\right)\right)^{2}<\infty
\end{aligned}
$$


where the trivial fact $(\alpha+\beta)^{2} \leq(\alpha+\beta)^{2}+(\alpha-\beta)^{2}=2\left(\alpha^{2}+\beta^{2}\right)$ for real numbers was employed.

From Step (1) of the proof of Proposition 3.3.1, it is already known that there exists a symplectic orthonormal basis $\left\{n_{l}, n_{l^{\prime}}\right\}=\left\{n_{L}\right\}$ of $H$ with $\left\{n_{l}\right\} \supseteq\left\{e_{k}\right\}$. For any element $n_{L}$ of this basis the quantity $\sigma\left(e_{k}, n_{L}\right)$ is nonzero for at most one choice of $k$, and since $\sum_{i, j} \lambda_{i j k}^{2}<\infty$, it must be the case that $n_{L} \in D(\Lambda)$. Hence, the linear operator $\Lambda$ is defined on the linear hull of $\left\{n_{K}\right\}$ and consequently is densely defined. The relation $\mathrm{R}(\Lambda) \subseteq P_{+} H^{2}$ is trivially fulfilled, because of the symmetry of $\lambda_{i j k}$. It has therefore been proven that $\Lambda \in \mathscr{L}$.

3. Since the sufficient condition of Lemma 3.2.10 is clearly satisfied, it suffices to demonstrate that equation $(3.2 .14)$ holds in order to conclude that $\Lambda \in \mathscr{L}_{C C R}$. For $f, g \in D(\Lambda)$ one verifies that

$$
\begin{aligned}
b(f, \Lambda g) & =b\left(f, \sum_{i, j} \sum_{k} \lambda_{i j k} \sigma\left(e_{k}, g\right) e_{i} \otimes_{e_{j}}\right)=\sum_{i, j} \sum_{k} \lambda_{i j k} \sigma\left(e_{k}, g\right) b\left(f, e_{i} \otimes e_{j}\right) \\
& =\sum_{j} \sum_{i} \sum_{k} \lambda_{i j k} \sigma\left(e_{k}, g\right) \sigma\left(f, e_{i}\right) e_{j}=\sum_{j} \sum_{k} \sum_{i} \lambda_{i j k} \sigma\left(e_{i}, g\right) \sigma\left(f, e_{k}\right) e_{j}
\end{aligned}
$$

and

$$
b(g, \Lambda f)=\sum_{j} \sum_{i} \sum_{k} \lambda_{i j k} \sigma\left(e_{k}, f\right) \sigma\left(g, e_{i}\right) e_{j}
$$

These two expressions are equal, if the sums $\sum_{i}$ and $\sum_{k}$ can be exchanged, and that will be the case if the double sum

$$
\sum_{i} \sum_{k} \lambda_{i j k} \sigma\left(e_{k}, f\right) \sigma\left(g, e_{i}\right)
$$

is absolutely convergent. To demonstrate that this is the case here, consider the sequences $\varphi$ and $\lambda_{i j}$ from the first part of this proof and also the sequences

$$
\phi \equiv\left(\left|\sigma\left(e_{1}, g\right)\right|,\left|\sigma\left(e_{2}, g\right)\right|,\left|\sigma\left(e_{3}, g\right)\right|, \ldots\right), \quad \lambda_{j} \equiv\left(\left\|\lambda_{1 j}\right\|_{\ell_{2}},\left\|\lambda_{2 j}\right\|_{\ell_{2}},\left\|\lambda_{3} j\right\|_{\ell_{2}}, \ldots\right) ;
$$

$\phi$ is an element of $\ell_{2}$ for the same reason that $\varphi$ is, and also $\lambda_{j}$ belongs to $\ell_{2}$, since $\sum_{i}\left\|\lambda_{i j}\right\|_{\ell_{2}}^{2}=\sum_{i} \sum_{k} \lambda_{i j k}^{2}<\infty$. Therefore, one has

$$
\infty>\|\varphi\|_{\ell_{2}}\left\langle\lambda_{j}, \varphi\right\rangle_{\ell_{2}}=\|\varphi\|_{\ell_{2}} \sum\left\|\lambda_{i}\right\|_{\ell_{2}}\left|\sigma\left(e_{i}, g\right)\right| \geq \sum_{i} \sum_{k}\left|\lambda_{i j k}\left\|\sigma\left(e_{k}, f\right)\right\| \sigma\left(e_{i}, g\right)\right|,
$$

where equation (3.3.3) was used in the last step. This demonstrates the desired absolute convergence, and $b(f, \Lambda g)=b(g, \Lambda f)$ follows. 
We next shall show that each $\Lambda \in \mathscr{L}_{C C R}$ has a maximal extension $\Lambda_{\max } \in$ $\mathscr{L}_{C C R}$. To this end we make the following definition.

Definition 3.3.3 For a given $\Lambda \in \mathscr{L}_{C C R}$ determine the quantities $\left\{e_{K}\right\}$ and $\lambda_{i j k}$ as in Proposition 3.3.1, and then define with these an operator via Proposition 3.3.2. The resulting operator will be denoted by $\Lambda_{\max }$.

The fact emerged in the proof of Proposition 3.3.1 that the basis $\left\{e_{K}\right\}$ and the numbers $\lambda_{i j k}$ are not necessarily determined uniquely by $\Lambda$. It shall be made clear that the operator $\Lambda_{\max }$ is independent of this choice of $\left\{e_{K}\right\}$ and $\lambda_{i j k}$. An immediate consequence of Proposition 3.3.2 is that $\Lambda_{\max }$ is an element of $\mathscr{L}_{C C R}$; moreover, for any $f \in D(\Lambda)$ we have

$$
\|\Lambda f\|^{2}=\sum_{i, j}\left(\sum_{k} \lambda_{i j k} \sigma\left(e_{k}, f\right)\right)^{2}<\infty,
$$

so that $f \in D\left(\Lambda_{\max }\right)$, and $\Lambda_{\max }$ is indeed an extension of $\Lambda$. We next see that $\Lambda_{\max }$ is a maximal extension of $\Lambda$ in the class $\mathscr{L}_{C C R}$.

Proposition 3.3.4. If $\Lambda^{\prime} \in \mathscr{L}_{C C R}$ is an extension of $\Lambda$, then $\Lambda^{\prime} \subset \Lambda_{\text {max }}$ Furthermore, $\Lambda_{\max }$ is a closed operator.

Proof. 1. First consider the case $\Lambda^{\prime} \supset \Lambda_{\max }$. If $f \in D\left(\Lambda^{\prime}\right)$, then set $\Lambda^{\prime} f=\sum_{I} e_{I}$ $\otimes f_{I}$, where $\left\{e_{I}\right\}$ is the symplectic orthonormal basis from which $\Lambda$ and $\Lambda_{\max }$ are constructed, and the vectors $f_{I} \in H$ are obtained from

$$
b\left(J e_{I}, \Lambda^{\prime} f\right)=\sum_{J} \sigma\left(J e_{I}, e_{J}\right) f_{J}=-\sum_{J} \delta_{I J} f_{J}=-f_{I}
$$

Since $J e_{I} \in D\left(\Lambda_{\max }\right)$, by assumption one also has $J e_{I} \in D\left(\Lambda^{\prime}\right)$, so that

$$
\begin{aligned}
f_{I} & =-b\left(J e_{I}, \Lambda^{\prime} f\right)=-b\left(f, \Lambda^{\prime} J e_{I}\right)=-b\left(f, \Lambda_{\max } J e_{I}\right) \\
& =-b\left(f, \sum_{m, n} \sum_{k} \lambda_{m n k} \sigma\left(e_{k}, J e_{I}\right) e_{m} \otimes e_{n}\right)=-\sum_{m, n} \sum_{k} \lambda_{m n k} \delta_{k I} \sigma\left(f, e_{m}\right) e_{n} .
\end{aligned}
$$

Therefore, $f_{i^{\prime}}=0$ and $f_{i}=\sum_{n} \sum_{m} \lambda_{m n i} \sigma\left(e_{m}, f\right) e_{n}$, which entails

$$
\left\|\Lambda^{\prime} f\right\|^{2}=\sum_{I}\left\|f_{I}\right\|^{2}=\sum_{i} \sum_{n}\left(\sum_{m} \lambda_{m n i} \sigma\left(e_{m}, f\right)\right)^{2} .
$$

Since this quantity must be finite, it follows that $f \in D\left(\Lambda_{\max }\right)$.

2. In general, one cannot assume that $D(\Lambda)$ or $D\left(\Lambda^{\prime}\right)$ contain a symplectic orthonormal basis, but, as has been argued before, there exists an orthonormal basis $\left\{k_{s}\right\}$ for the scalar product in $H$ such that $J k_{s} \in D(\Lambda) \subset D\left(\Lambda^{\prime}\right)$. Using this 
basis we write as above

$$
\begin{aligned}
\Lambda_{\max } f & =\sum_{s} k_{s} \otimes l_{\max , s}(f)=\sum_{s} l_{\text {max }, s}(f) \otimes k_{s}, \\
l_{\text {max }, s}(f) & =-b\left(J k_{s}, \Lambda_{\max } f\right), \quad f \in D\left(\Lambda_{\max }\right)
\end{aligned}
$$

and

$$
\Lambda^{\prime} g=\sum_{s} k_{s} \otimes l_{s}^{\prime}(g)=\sum_{s} l_{s}^{\prime}(g) \otimes k_{s}, \quad l_{s}^{\prime}(g)=-b\left(k_{s}, \Lambda^{\prime} g\right), \quad g \in D\left(\Lambda^{\prime}\right)
$$

Since $f \in D(\Lambda)$ entails $\Lambda_{\max } f=\Lambda f=\Lambda^{\prime} f, l_{\max , s}(\cdot)$ and $l_{s}^{\prime}(\cdot)$ coincide on $D(\Lambda)$. Because $l_{\text {max }, s}(f)=-b\left(f, \Lambda_{\max } J k_{s}\right)$ and $l_{s}^{\prime}(g)=-b\left(g, \Lambda^{\prime} J k_{s}\right)$, the linear maps $f \mapsto$ $l_{\text {max }, s}(f)$ and $g \mapsto l_{s}^{\prime}(g)$ are continuous. Thus, since the continuous extension of a linear, continuous, densely defined operator is unique, $l_{\max , s}(\cdot)$ and $l_{s}^{\prime}(\cdot)$ are restrictions of the same continuous map $l_{s}(\cdot)$ defined on all of $H$.

If $f \in D\left(\Lambda_{\max }\right)$ and $g \in D\left(\Lambda^{\prime}\right)$, then the sum $\sum_{s} k_{s} \otimes l_{s}(f+g)$ converges strongly, since by construction $\sum_{s} k_{s} \otimes l_{s}(f)$ and $\sum_{s} k_{s} \otimes l_{s}(g)$ converge. One may therefore define an operator $\tilde{\Lambda}$ by

$$
\begin{gathered}
\widetilde{\Lambda} h \equiv \sum_{s} k_{s} \otimes l_{s}(h)=\sum_{s} l_{s}(h) \otimes k_{s}, \\
h \in D(\widetilde{\Lambda}) \equiv D\left(\Lambda_{\max }\right)+D\left(\Lambda^{\prime}\right)=\left\{f+g \mid f \in D\left(\Lambda_{\max }\right), g \in D\left(\Lambda^{\prime}\right)\right\} .
\end{gathered}
$$

One clearly has $\tilde{\Lambda} \supset \Lambda_{\max }$ and $\tilde{\Lambda} \supset \Lambda^{\prime}$.

It will next be shown that $\tilde{\Lambda} \in \mathscr{L}_{C C R}$. First it is noted that since $\tilde{\Lambda}(h)=\tilde{\Lambda}(f$ $+g)=\Lambda_{\max } f+\Lambda^{\prime} g \in P_{+} H^{2}$, one has $\tilde{\Lambda} \in \mathscr{L}$. With $f, g \in D(\widetilde{\Lambda})$ one has

$$
B(\widetilde{\Lambda} f, \widetilde{\Lambda} g)=\sum_{r, s} \sigma\left(l_{r}(f), l_{s}(g)\right) k_{r} \otimes k_{s}
$$

and

$$
b(f, \tilde{\Lambda} g)=\sum_{s} \sigma\left(f, l_{s}(g)\right) k_{s}
$$

Choosing two sequences $\left(f_{m}\right)$ and $\left(g_{n}\right)$ from $D(\Lambda)$, which converge to $f$, resp. $g$ $\in D(\widetilde{\Lambda})$, it follows from $B\left(\Lambda f_{m}, \Lambda g_{n}\right)=0$ that $\sigma\left(l_{r}\left(f_{m}\right), l_{s}\left(g_{n}\right)\right)=0$ and from $b\left(f_{m}, \Lambda g_{n}\right)=b\left(g_{n}, \Lambda f_{m}\right)$ that $\sigma\left(f_{m}, l_{s}\left(g_{n}\right)\right)=\sigma\left(g_{n}, l_{s}\left(f_{m}\right)\right)$, for every $r, s, m$ and $n$. Thus,

$$
\begin{aligned}
\sigma\left(l_{r}(f), l_{s}(g)\right) & =\sigma\left(l_{r}\left(\lim _{m} f_{m}\right), l_{s}(g)\right)=\sigma\left(\lim _{m} l_{r}\left(f_{m}\right), l_{s}(g)\right) \\
& =\lim _{m} \sigma\left(l_{r}\left(f_{m}\right), l_{s}(g)\right)=\lim _{m} \lim _{n} \sigma\left(l_{r}\left(f_{m}\right), l_{s}\left(g_{n}\right)\right)=0
\end{aligned}
$$


entails $B(\widetilde{\Lambda} f, \widetilde{\Lambda} g)=0$, and

$$
\sigma\left(f, l_{s}(g)\right)=\lim _{m} \lim _{n} \sigma\left(f_{m}, l_{s}\left(g_{n}\right)\right)=\lim _{m} \lim _{n} \sigma\left(g_{n}, l_{s}\left(f_{m}\right)\right)=\sigma\left(g, l_{s}(f)\right)
$$

entails $b(f, \widetilde{\Lambda} g)=b(g, \widetilde{\Lambda} f)$. Hence one has $\widetilde{\Lambda} \in \mathscr{L}_{C C R}$. From the first step of this proof, it then follows from $\widetilde{\Lambda} \supset \Lambda_{\max }$ the equality $\widetilde{\Lambda}=\Lambda_{\max }$, so that $\Lambda^{\prime} \subset \widetilde{\Lambda}=\Lambda_{\max }$.

3. Finally, one turns to the closedness of $\Lambda_{\max }$. To this end, one considers $\Lambda_{\max }$ in the form

$$
D\left(\Lambda_{\max }\right)=\left\{f \in H \mid \sum_{\imath}\left\|h_{\imath}(f)\right\|^{2}<\infty\right\}, \quad \Lambda_{\max } f=\sum_{\imath} e_{\imath} \otimes h_{\imath}(f)
$$

with the continuous functions $h_{\imath}(f)=-b\left(J e_{l}, \Lambda_{\max } f\right)=\sum_{j} \sum_{k} \lambda_{i j k} \sigma\left(e_{k}, f\right) e_{j}$. Let $\left(f_{n}\right)$ be a sequence in $D\left(\Lambda_{\max }\right)$ which converges to $f \in H$ so that $\left(\Lambda_{\max } f_{n}\right)$ converges to $F \in P_{+} H^{2}$. Set $F=\sum_{I} e_{I} \otimes g_{I}$, so that $g_{\imath}=\lim _{n} h_{\imath}\left(f_{n}\right)$ and $g_{i^{\prime}}=0$, since

$$
\Lambda_{\max } f_{n}=\sum_{\imath} e_{\imath} \otimes h_{\imath}\left(f_{n}\right) \stackrel{n \rightarrow \infty}{\longrightarrow} F=\sum_{I} e_{I} \otimes g_{I}
$$

But because of the continuity of $h_{\imath}(\cdot), h_{i}\left(f_{n}\right)$ converges to $h_{\imath}(f)$. Therefore, $g_{\imath}$ $=h_{i}(f)$, so $F=\sum_{i e_{\imath}} \otimes h_{\imath}(f)$, which implies $\sum_{i}\left\|h_{\imath}(f)\right\|^{2}=\|F\|^{2}$ and thus also $f \in$ $D\left(\Lambda_{\max }\right)$ and $\Lambda_{\max } f=F$, concluding the proof.

From the proof of this last proposition we see that for every $\Lambda \in \mathscr{L}_{C C R}$ there is a unique maximal extension $\Lambda_{\max } \in \mathscr{L}_{C C R}$. Moreover, each such $\Lambda$ is closable, since it possesses a closed extension (namely $\Lambda_{\max }$ ), and, of course, $\bar{\Lambda} \subset \overline{\Lambda_{\max }}=$ $\Lambda_{\max }$. It may be the case that $\widetilde{\Lambda}=\Lambda_{\max }$, but this has not been determined.

Since $\Lambda_{\max }$ uniquely exists, it is henceforth admissible to consider $\Lambda$ as being defined on the symplectic orthonormal basis $\left\{e_{K}\right\}$, where it takes the values $\Lambda e_{k}=0$ and $\Lambda e_{k^{\prime}}=\sum_{i, j} \lambda_{i j k} e_{i} \otimes e_{j}$. We can now finally state what we mean by the standard form of a canonical quadratic transformation $\Phi(f) \mapsto \Phi_{\Lambda}(f)$ in terms of the field operators $\Phi\left(e_{K}\right)$, where $\left\{e_{K}\right\}$ is a symplectic orthonormal basis in which $\Lambda$ takes the form (3.3.1).

$$
q_{k} \equiv \Phi\left(e_{k}\right) \mapsto \Phi_{\Lambda}\left(e_{k}\right)=\overline{\Phi\left(e_{k}\right)+\Psi\left(\Lambda e_{k}\right)}=q_{k},
$$

and

$$
\begin{aligned}
p_{k} \equiv \Phi\left(e_{k^{\prime}}\right) \mapsto \Phi_{\Lambda}\left(e_{k^{\prime}}\right) & =\overline{\Phi\left(e_{k^{\prime}}\right)+\Psi\left(\Lambda e_{k^{\prime}}\right)} \\
& =\overline{p_{k}+\Psi\left(\sum_{i, j} \lambda_{i j k} e_{i} \otimes_{e_{j}}\right)}
\end{aligned}
$$




$$
\begin{aligned}
& =\overline{p_{k}+\sum_{i, j} \lambda_{i j k} \Psi\left(e_{i} \otimes e_{j}\right)} \\
& =\overline{p_{k}+\sum_{i, j} \lambda_{i j k}\left(: q_{i} q_{j}:\left.\right|_{\mathscr{F}_{0}}\right)} .
\end{aligned}
$$

In short, the standard form of a canonical quadratic transformation is

$$
q_{k} \mapsto q_{k}, \quad p_{k} \mapsto \overline{p_{k}+\sum_{i, j} \lambda_{i j k}\left(: q_{i} q_{j}:\left.\right|_{\mathscr{F}_{0}}\right)}
$$

The above results demonstrate that every canonical quadratic transformation can be written in this standard form, so that the apparently special case in troduced in [22] was already perfectly general.

\section{Unitary Equivalence Characterized}

In the previous section, we have characterized the canonical quadratic transformations, but in general there is certainly no guarantee that a representation of the CCR can be exponentiated to obtain a representation of the corresponding Weyl algebra. The first section of this chapter is dedicated to the proof that, in fact, representations of the CCR which are obtained from the Fock representation via canonical quadratic transformations can be exponentiated. Then we present the proof of the main theorem of this paper, which characterizes those quadratic representations which are unitarily equivalent to the Fock representation. In particular, we shall prove the following theorem.

Theorem 4.1. Let $\Lambda \in \mathscr{L}_{C C R}$ and let $\Phi_{\Lambda}(f)$ be the corresponding representation of the CCR. $\Phi_{\Lambda}(f)$ is unitarily equivalent to the Fock representation $\Phi(f)$ if and only if the map $\Lambda: H \mapsto H \otimes H$ is Hilbert-Schmidt. Moreover, the corresponding Weyl representation $W_{\Lambda}(f)$ is unitarily equivalent to the Fock representation $W(f)$ if and only if the map $\Lambda: H \mapsto H \otimes H$ is Hilbert-Schmidt.

\section{§4.1. Quadratic Transformations and Weyl Algebras}

First we shall prove that any canonical quadratic representation can be exponentiated to obtain a representation of the Weyl algebra.

Theorem 4.1.1. Let $\Lambda \in \mathscr{L}_{C C R}$ and define the unitary operators $W_{\Lambda}(f), f \in$ $D(\Lambda)$, on $\mathscr{F}_{+}(\mathscr{H})$ by $W_{\Lambda}(f) \equiv e^{i \Phi_{\Lambda}(f)}$. Then for any $f, g \in D(\Lambda)$,

(a) $W_{\Lambda}(-f)=W_{\Lambda}(f)^{*}$ and (b) $W_{\Lambda}(f) W_{\Lambda}(g)=e^{-\imath \sigma(f, g) / 2} W_{\Lambda}(f+g)$. 
Proof. 1. The unitarity of $W_{\Lambda}(f)$ is an immediate consequence of Proposition 3.1.3, and assertion (a) is clear: $W_{\Lambda}(-f)=e^{i \Phi_{\Lambda}(-f)}=e^{-\imath \Phi_{\Lambda}(f)}=W_{\Lambda}(f) *$. Thus, only assertion (b) is nontrivial. The proof of (b) uses analytic vectors and the exponential series for $e^{\imath \Phi_{\Lambda}(f)}$. An analogous proof for the Segal field $\Phi$ instead of $\Phi_{\Lambda}$ is in [21] (see Theorem X.41).

If $f=0$ or $g=0$, then the Weyl relations (b) obtain trivially. Hence, it shall be assumed that $f \neq 0$ and $g \neq 0$ are chosen from $D(\Lambda)$. Define the functional $\alpha \in$ $D(\Lambda)^{\prime}$ by $\alpha(h) \equiv\|h\|+2\|\Lambda h\|$, and let $k$ be a natural number so that $k>4 \sqrt{2} \max$ $\{\alpha(f), \alpha(g)\}$ and $0<\beta \equiv \frac{1}{k}<\frac{1}{4 \sqrt{2} \max \{\alpha(f), \alpha(g)\}}$. Finally, let $\varphi=\left(\varphi^{(0)}, \varphi^{(1)}\right.$, $\left.\ldots, \varphi^{\left(n_{\varphi}\right)}, 0, \ldots\right)$ be a finite-particle vector.

From Proposition 3.1 .3 one knows that the series $\sum_{m=0}^{\infty} \frac{t^{m}}{m !}\left\|\Phi_{\Lambda}(\beta f+\beta g)^{m} \varphi\right\|$ converges for all $t$ such that $0 \leq t<1 / 2 \sqrt{2} \alpha(\beta f+\beta g)$ and that $\varphi$ is an analytic vector for $\Phi_{\Lambda}(\beta f+\beta g)$. Thus one has

$$
e^{\imath \Phi_{\Lambda}(\beta f+\beta g)} \varphi=\sum_{m=0}^{\infty} \frac{i^{m}}{m !} \Phi_{\Lambda}(\beta f+\beta g)^{m} \varphi
$$

since $\beta$ has been chosen to satisfy $|i|<\frac{1}{2 \sqrt{2} \alpha(\beta f+\beta g)}$. Therefore, one may write

$$
\begin{aligned}
e^{-\imath \sigma(\beta f, \beta g) / 2} W_{\Lambda}(\beta f+\beta g) \varphi & =e^{-\imath \sigma(\beta f, \beta g) / 2} \sum_{m=0}^{\infty} \frac{i^{m}}{m !} \Phi_{\Lambda}(\beta f+\beta g)^{m} \varphi \\
& =\sum_{n=0}^{\infty} \sum_{m=0}^{\infty} \frac{i^{m+n}}{n ! m !}\left(-\frac{1}{2}\right)^{n} \beta^{2 n+m} \sigma(f, g)^{n}\left(\Phi_{\Lambda}(f)+\Phi_{\Lambda}(g)\right)^{m} \varphi
\end{aligned}
$$

On the other hand, $\varphi$ is also an analytic vector of $\Phi_{\Lambda}(\beta g)$, and

$$
e^{i \Phi_{\Lambda}(\beta g)} \varphi=\sum_{m=0}^{\infty} \frac{i^{m}}{m !} \Phi_{\Lambda}(\beta g)^{m} \varphi
$$

From the proof of Proposition 3.1.3 it is already established that

$$
\left\|\Phi_{\Lambda}(f)^{n} \phi\right\| \leq 2^{n / 2} \alpha(f)^{n}\left[\left(n_{\psi}+1\right)\left(n_{\psi}+2\right) \cdots\left(n_{\psi}+2 n\right)\right]^{1 / 2}\|\phi\|
$$

where $\alpha(f) \equiv\|f\|+2\|\Lambda f\|$. Thus one obtains

$$
\begin{aligned}
\left\|\Phi_{\Lambda}(f)^{n} \Phi_{\Lambda}(g)^{m} \varphi\right\| & \leq 2^{n / 2} \alpha(f)^{n}\left[\left(n_{\varphi}+2 m+1\right) \cdots\left(n_{\varphi}+2 m+2 n\right)\right]^{1 / 2}\left\|\Phi_{\Lambda}(g)^{m} \varphi\right\| \\
& \leq 2^{n / 2} \alpha(f)^{n} 2^{m / 2} \alpha(g)^{m}\left[\left(n_{\varphi}+1\right) \cdots\left(n_{\varphi}+2 m\right)\right.
\end{aligned}
$$




$$
\begin{aligned}
& \left.\cdot\left(n_{\varphi}+2 m+1\right) \cdots\left(n_{\varphi}+2 m+2 n\right)\right]^{1 / 2}\|\varphi\| \\
& \leq 2^{(n+m) / 2} \alpha(f)^{n} \alpha(g)^{m} \sqrt{\left(n_{\varphi}+2 m+2 n\right) !}\|\varphi\| \\
& \leq 2^{(n+m) / 2} \alpha(f)^{n} \alpha(g)^{m}\left(n_{\varphi}+4 n\right) !^{1 / 4}\left(n_{\varphi}+4 m\right) !^{1 / 4}\|\varphi\| .
\end{aligned}
$$

The last inequality follows from the straightforward estimate $(n+m)$ ! $\leq \sqrt{(2 n) !(2 m) !}$. Hence one may bound $\sum_{n, m=0}^{\infty} \frac{t^{n}}{n ! m !}\left\|\Phi_{\Lambda}(\beta f)^{n} \Phi_{\Lambda}(\beta g)^{m} \varphi\right\|$ from above by

$$
\left(\sum_{n=0}^{\infty} \frac{2^{n / 2} t^{n}}{n !} \alpha(\beta f)^{n}\left(n_{\varphi}+4 n\right) !^{1 / 4}\right)\left(\sum_{m=0}^{\infty} \frac{2^{m / 2}}{m !} \alpha(\beta g)^{m}\left(n_{\varphi}+4 m\right) !^{1 / 4}\right)\|\varphi\|
$$

Applying again the ratio test to, e.g., the second of the series in this product, one obtains

$$
\begin{gathered}
\frac{\left(2^{m / 2} / m !\right) \alpha(\beta g)^{m}\left(n_{\varphi}+4 m\right) !^{1 / 4}}{\left(2^{(m-1) / 2} /(m-1) !\right) \alpha(\beta g)^{m-1}\left(n_{\varphi}+4 m-4\right) !^{1 / 4}} \\
=\sqrt{2} \alpha(\beta g) \frac{1}{m}\left[\left(n_{\varphi}+4 m-3\right) \cdots\left(n_{\varphi}+4 m\right)\right]^{1 / 4} \\
\leq \sqrt{2} \alpha(\beta g) \frac{1}{m}\left(n_{\varphi}+4 m\right) \underset{m \rightarrow \infty}{\rightarrow} 4 \sqrt{2} \alpha(\beta g) .
\end{gathered}
$$

After a similar argument for the first of the series in the product, one may thereby conclude that the double series (4.1.1) converges whenever $4 \sqrt{2} \beta \alpha(f) t$ $<1$ and $4 \sqrt{2} \beta \alpha(g)<1$. The latter condition is satisfied by the specified choice of $\beta$. Thus one may conclude that (4.1.1) converges if $t<\frac{1}{4 \sqrt{2} \beta \alpha(f)}$, where also the case $t=1$ is included. Hence for arbitrary $n$,

$$
\sum_{m=0}^{\infty} \frac{1}{m !}\left\|\Phi_{\Lambda}(\beta f)^{n} \Phi_{\Lambda}(\beta g)^{m} \varphi\right\|<\infty
$$

from which one may conclude that $\sum_{m=0}^{\infty} \frac{i^{m}}{m !} \Phi_{\Lambda}(\beta f)^{n} \Phi_{\Lambda}(\beta g)^{m} \varphi$ converges. Since $\Phi_{\Lambda}(\beta f)^{n}$ is self-adjoint and therefore closed, it follows from this convergence that of $\sum_{m=0}^{\infty} \frac{i^{m}}{m !} \Phi_{\Lambda}(\beta g)^{m} \varphi$, which entails that $W_{\Lambda}(\beta g) \varphi$ is in the domain of definition of $\Phi_{\Lambda}(\beta f)^{n}$ and also that

$$
\Phi_{\Lambda}(\beta f)^{n} W_{\Lambda}(\beta g) \varphi=\sum_{m=0}^{\infty} \frac{i^{m}}{m !} \Phi_{\Lambda}(\beta f)^{n} \Phi_{\Lambda}(\beta g)^{m} \varphi
$$


The above estimates imply that $\mathrm{W}_{\Lambda}(\beta g) \varphi$ is in fact an analytic vector for $\Phi_{\Lambda}(\beta f)$, and one may use the exponential series again, i.e.

$$
e^{i \Phi_{\Lambda}(\beta f)} e^{i \Phi_{\Lambda}(\beta g)} \varphi=\sum_{n, m=0}^{\infty} \frac{i^{n+m}}{n ! m !} \beta^{n+m} \Phi_{\Lambda}(f)^{n} \Phi_{\Lambda}(g)^{m} \varphi
$$

Since the double series (4.1.1) and (4.1.2) are both absolutely convergent, one can arbitrarily re-order the series without changing the sum with the aim to compare terms of the same degree in $\beta$. Then, in the same manner as one establishes the validity of the Baker-Hausdorff formula $\left(e^{A} e^{B}=e^{[A, B] / 2} e^{A+B}\right.$ for $[A, B]$ $\in \mathbf{C}$ ), one verifies the equality of (4.1.1) and (4.1.2). For any $\varphi \in \mathscr{F}_{0}$ one has proven the validity of the equation $W_{\Lambda}(\beta f) W_{\Lambda}(\beta g) \varphi=e^{-\frac{i}{2} \sigma(\beta f, \beta g)} W_{\Lambda}(\beta f+\beta g) \varphi$. Since the Weyl operators are unitary, this relation is also fulfilled for all other vectors in Fock space.

2. In order to eliminate the quantity $\beta$, one writes

$$
W_{\Lambda}(f)=W_{\Lambda}(k \beta f)=e^{i k \Phi_{\Lambda}(\beta f)}=W_{\Lambda}(\beta f)^{k} \text { and } W_{\Lambda}(f) W_{\Lambda}(g)=W_{\Lambda}(\beta f)^{k} W_{\Lambda}(\beta g)^{k}
$$

But

$$
\begin{aligned}
W_{\Lambda}(\beta f) W_{\Lambda}(\beta g) & =e^{-i \sigma(\beta f, \beta g) / 2} W_{\Lambda}(\beta f+\beta g) \\
& =e^{-i \sigma(\beta f, \beta g)} e^{-i \sigma(\beta g, \beta f) / 2} W_{\Lambda}(\beta g+\beta f) \\
& =e^{-i \sigma(\beta f, \beta g)} W_{\Lambda}(\beta g) W_{\Lambda}(\beta f)
\end{aligned}
$$

Thus the product $W_{\Lambda}(f) W_{\Lambda}(g)$ can be rewritten:

$$
W_{\Lambda}(f) W_{\Lambda}(g)=W_{\Lambda}(\beta f)^{k-1} e^{-i \sigma(\beta f, \beta g)} W_{\Lambda}(\beta g) W_{\Lambda}(\beta f) W_{\Lambda}(\beta g)^{k-1}
$$

By repeating this commutation one can manipulate $W_{\Lambda}(f) W_{\Lambda}(g)$ until only the products $W_{\Lambda}(\beta f) W_{\Lambda}(\beta g)$ arise. Every such commutation yields a factor $e^{-i \sigma(\beta f, \beta g)}$, and the required number of commutations is $(k-1)+(k-2)+\cdots+(k$ $-(k-1))=\frac{1}{2} k(k-1)$. In this manner one obtains the desired result:

$$
\begin{aligned}
W_{\Lambda}(f) W_{\Lambda}(g) & =e^{-i \sigma(\beta f, \beta g) \cdot \frac{1}{2} k(k-1)}\left(W_{\Lambda}(\beta f) W_{\Lambda}(\beta g)\right)^{k} \\
& =e^{-\frac{i}{2} k(k-1) \sigma(\beta f, \beta g)} e^{-\frac{i}{2} k \sigma(\beta f, \beta g)} W_{\Lambda}(\beta f+\beta g)^{k} \\
& =e^{-\frac{i}{2} k^{2} \sigma^{(\beta f, \beta g)} W_{\Lambda}(k(\beta f+\beta g))=e^{-\frac{i}{2} \sigma(f, g)} W_{\Lambda}(f+g) .} .
\end{aligned}
$$

Consider the $C^{*}$-subalgebra $\mathscr{A}(D(\Lambda))$ of the CCR-algebra $\mathscr{A}(H, \sigma)$ generated by the set $\{W(f) \in \mathscr{A}(H, \sigma) \mid f \in D(\Lambda)\}$. If $\Lambda$ is bounded, then $D(\Lambda)=H$ and 
$\mathscr{A}(D(\Lambda))=\mathscr{A}(H, \sigma)$. But if $\Lambda$ is unbounded, we may consider it maximally defined by replacing it with $\Lambda_{\text {max }}$. The resulting algebra $\mathscr{A}(D(\Lambda))$ is then ([2], Theorem 5.2.9) a proper subalgebra of $\mathscr{A}(H, \sigma)$. By the uniqueness of the CCR-algebra over a preHilbert space, the algebra $\mathscr{A}(D(\Lambda))$ is just the CCR-algebra over $(D(\Lambda), \sigma)$. On the other hand, the $C^{*}$-algebra $\mathscr{A}_{\Lambda}(D(\Lambda))$ generated by the operators $W_{\Lambda}(f)=e^{i \Phi_{\Lambda}(f)}, f \in D(\Lambda)$ on Fock space is, by Theorem 4.1.1 and [2], Theorem 5.2.8, isomorphic to $\mathscr{A}(D(\Lambda))$, i.e. there exists a unique $C^{*}$-isomorphism $\pi_{\Lambda}$ from $\mathscr{A}(D(\Lambda))$ onto $\mathscr{A}_{\Lambda}(D(\Lambda))$, such that $\pi_{\Lambda}(W(f))=W_{\Lambda}(f)$ for all $f \in D(\Lambda)$.

Corollary 4.1.2. For any $\Lambda \in \mathscr{L}_{C C R}, \pi_{\Lambda}$ is a faithful, regular, irreducible representation of the CCR-algebra $\mathscr{A}(D(\Lambda))$.

Proof. Since the other assertions are obvious, only the proof of the claim of irreducibility will be sketched. If $A \in \mathscr{B}\left(\mathscr{F}_{+}(\mathscr{H})\right)$ commutes with all elements of $\mathscr{A}_{\Lambda}(D(\Lambda))$, then it commutes with every $\Phi_{\Lambda}(f)$. Since $q_{k}$ and $p_{k}$ can be expressed in terms of the $\Phi_{\Lambda}\left(e_{J}\right)$ (which is clear from the standard form (3.3.4)), $A$ commutes also with all $q_{k}$ and $p_{k}$. Since the Fock representation is irreducible, $A$ must be trivial.

We comment that the representation $\pi_{\Lambda}$ is not quasifree in general. In fact, since $\left\langle\Omega_{0}, \Phi_{\Lambda}(f) \Omega_{0}\right\rangle=0$, the truncated three-point-function equals

$$
\left\langle\Omega_{0}, \Phi_{\Lambda}\left(f_{1}\right) \Phi_{\Lambda}\left(f_{2}\right) \Phi_{\Lambda}\left(f_{3}\right) \Omega_{0}\right\rangle
$$

and the truncated four-point-function is

$$
\begin{aligned}
& \left\langle\Omega_{0}, \Phi_{\Lambda}\left(f_{1}\right) \Phi_{\Lambda}\left(f_{2}\right) \Phi_{\Lambda}\left(f_{3}\right) \Phi_{\Lambda}\left(f_{4}\right) \Omega_{0}\right\rangle \\
& -\left\langle\Omega_{0}, \Phi_{\Lambda}\left(f_{1}\right) \Phi_{\Lambda}\left(f_{2}\right) \Omega_{0}\right\rangle\left\langle\Omega_{0}, \Phi_{\Lambda}\left(f_{3}\right) \Phi_{\Lambda}\left(f_{4}\right) \Omega_{0}\right\rangle \\
& -\left\langle\Omega_{0}, \Phi_{\Lambda}\left(f_{1}\right) \Phi_{\Lambda}\left(f_{3}\right) \Omega_{0}\right\rangle\left\langle\Omega_{0}, \Phi_{\Lambda}\left(f_{2}\right) \Phi_{\Lambda}\left(f_{4}\right) \Omega_{0}\right\rangle \\
& -\left\langle\Omega_{0}, \Phi_{\Lambda}\left(f_{1}\right) \Phi_{\Lambda}\left(f_{4}\right) \Omega_{0}\right\rangle\left\langle\Omega_{0}, \Phi_{\Lambda}\left(f_{2}\right) \Phi_{\Lambda}\left(f_{3}\right) \Omega_{0}\right\rangle .
\end{aligned}
$$

If one chooses $\Lambda f \equiv \lambda \sigma(e, f) e \otimes e$ with $\lambda \in \mathbb{R} \backslash\{0\}$ and $e \in H$ a unit vector, and sets $f_{1}=f_{2}=e, f_{3}=f_{4}=J e$, then one finds that neither of these truncated functions vanishes. Of course, that does not exclude the possibility that $\pi_{\Lambda}$ is unitarily equivalent to a quasifree representation. But note that since quadratic representations are irreducible and since pure quasifree states are Fock states [14], $\pi_{\Lambda}$ is unitarily equivalent to a quasifree representation if and only if it is unitarily equivalent to a Fock representation (though, of course, not necessarily the original Fock representation). It appears that, in fact, a quadratic representation is unitarily equivalent to a quasifree representation if and only if both are unitarily equivalent to the original Fock representation [23]. Moreover, $\pi_{\Lambda}$ 
is unitarily equivalent to a coherent representation if and only if once again both are unitarily equivalent to the orizinal Fock representation [23]. These results will be proven elsewhere.

\section{§4.2. Unitary Equivalence Implies $\Lambda$ Hilbert-Schmidt}

Though, as has just been shown, the algebras $\mathscr{A}(D(\Lambda))$ and $\mathscr{A}_{\Lambda}(D(\Lambda))$ are isomorphic, what is of primary physical concern is whether they are unitarily equivalent. (Unfortunately, it is a common mistake in the physics literature to overlook the difference between these two notions.) To specify exactly what we shall mean below, we shall say that the transformation $\Phi(f) \rightarrow \Phi_{\Lambda}(f)$ is unitarily implementable (or the two representations $\Phi(f)$ and $\Phi_{\Lambda}(f)$ are unitarily equivalent), when there exists a unitary operator $U: \mathscr{F}_{+}(\mathscr{H}) \mapsto \mathscr{F}_{+}(\mathscr{H})$ such that $U \Phi(f) U^{*} \phi=\Phi_{\Lambda}(f) \phi$ for all $f \in D(\Lambda)$ and all $\phi \in D\left(\Phi_{\Lambda}(f)\right)$. (Recall that we have defined the operators $\Phi(f)$ and $\Phi_{\Lambda}(f)$ to be closed and have shown that they are, in fact, self-adjoint.) This entails, in particular, that $U^{*} D\left(\Phi_{\Lambda}(f)\right)$ $\subset D(\Phi(f))$, for all $f \in D(\Lambda)$, and these domains of definition can indeed depend upon the choice of $f$. Note that by Theorem 4.1.1, $\Phi(f) \rightarrow \Phi_{\Lambda}(f)$ is unitarily implementable if and only if $U W(f) U^{*}=W_{\Lambda}(f)$ for all $f \in D(\Lambda)$. First we consider the proof of the following lemma.

Lemma 4.2.1. Let $\Lambda \in \mathscr{L}_{C C R}$. If the canonical transformation $\Phi(f) \rightarrow \Phi_{\Lambda}(f)$ is unitarily implementable, then $\Lambda$ is bounded.

Proof. 1. Let $U$ be the unitary such that $U \Phi(f) U^{*}=\Phi_{\Lambda}(f)$ for all $f \in$ $D(\Lambda)$. Thus the set $U \mathscr{F}_{0}$ is dense in $\mathscr{F}_{+}(\mathscr{H})$ and is contained in the domain of definition of $\Phi_{\Lambda}(f)$. Using the closedness of $\Phi_{\Lambda}(f)$, let $\phi \in U \mathscr{F}_{0} \subset D\left(\Phi_{\Lambda}(f)\right)$ and $\left\{\phi_{k}\right\} \subset \mathscr{F}_{0}$ converge to $\phi$ in such a manner that $\Phi_{\Lambda}(f) \phi_{k} \rightarrow \Phi_{\Lambda}(f) \phi$. Then, since $\Psi(\Lambda f)$ is quadratic in the creation and annihilation operators, it is clear that

$$
P_{n} \Phi_{\Lambda}(f) \phi=\lim _{k \rightarrow \infty} P_{n} \Phi_{\Lambda}(f) \phi_{k}=\lim _{k \rightarrow \infty} P_{n} \Phi_{\Lambda}(f)\left(P_{n-2}+\cdots+P_{n+2}\right) \phi_{k}
$$

But since $P_{n} \Phi_{\Lambda}(f)$ is closed, it follows that $P_{n} \Phi_{\Lambda}(f) \phi=P_{n} \Phi_{\Lambda}(f)\left(P_{n-2}+\cdots+\right.$ $\left.P_{n+2}\right) \psi$. Arguing similarly on the components $\Phi(f)$ and $\Phi(\Lambda f)$ and using the comments following equation (3.1.3), one obtains

$$
\begin{aligned}
P_{n} U \Phi(f) U^{*} \phi & =P_{n} \Phi_{\Lambda}(f) \phi=P_{n}(\Phi(f)+\Psi(\Lambda f))\left(P_{n-2}+\cdots+P_{n+2}\right) \psi \\
& =P_{n} \Phi(f)\left(P_{n-1}+P_{n+1}\right) \psi+P_{n} \Psi(\Lambda f)\left(P_{n-2}+P_{n}+P_{n+2}\right) \psi
\end{aligned}
$$

in particular, for $n=2$ : 


$$
P_{2} U \Phi(f) U^{*} \phi-P_{2} \Phi(f)\left(P_{1}+P_{3}\right) \phi=P_{2} \Psi(\Lambda f)\left(P_{0}+P_{2}+P_{4}\right) \psi
$$

But since $U^{*} \phi \in \mathscr{F}_{0}$ and $\left(P_{1}+P_{3}\right) \phi \in \mathscr{F}_{0}$, the maps $f \mapsto \Phi(f) U^{*} \phi$ and $f \mapsto \Phi(f)\left(P_{1}\right.$ $\left.+P_{3}\right) \psi$ are continuous, and thus so are the maps $f \mapsto P_{2} U \Phi(f) U^{*} \psi$ and $f \mapsto$ $P_{2} \Phi(f)\left(P_{1}+P_{3}\right) \psi$. Hence it follows that the map $f \mapsto P_{2} \Psi(\Lambda f)\left(P_{0}+P_{2}+P_{4}\right) \phi$ from $D(\Lambda)$ to $\mathscr{F}_{+}(H)$ is continuous.

2. Consider now the expression $P_{2} \Psi(\Lambda f)\left(P_{0}+P_{2}+P_{4}\right) \psi$. One has

$$
\begin{aligned}
\left\|P_{2} \Psi(\Lambda f)\left(P_{0}+P_{2}+P_{4}\right) \phi\right\| & =\left\|P_{2} \Psi(\Lambda f)\left(P_{0}+P_{2}+P_{4}\right) \phi\right\|+\left\|-P_{2} \Psi(\Lambda f)\left(P_{2}+P_{4}\right) \phi\right\| \\
& \quad-\left\|P_{2} \Psi(\Lambda f)\left(P_{2}+P_{4}\right) \phi\right\| \\
& \geq\left\|P_{2} \Psi(\Lambda f)\left(P_{0}+P_{2}+P_{4}\right) \phi-P_{2} \Psi(\Lambda f)\left(P_{2}+P_{4}\right) \phi\right\| \\
& \quad-\left\|P_{2} \Psi(\Lambda f)\left(P_{2}+P_{4}\right) \phi\right\| \\
= & \left\|P_{2} \Psi(\Lambda f) P_{0} \psi\right\|-\left\|P_{2} \Psi(\Lambda f)\left(P_{2}+P_{4}\right) \psi\right\| .
\end{aligned}
$$

Now, of course, $P_{0} \phi=\left(\phi^{(0)}, 0,0, \ldots\right)=\phi^{(0)} \Omega_{0}$, where $\phi^{(0)} \in \mathbb{C}$. Since $\Lambda \in \mathscr{L}_{C C R}$, Theorem 3.3.1 yields $\Lambda=\sum_{i, j} \sum_{k} \lambda_{i j k} \sigma\left(e_{k}, \cdot\right) e_{i} \otimes \mathrm{e}_{j}$, and one finds

$$
\begin{aligned}
P_{2} \Psi(\Lambda f) P_{0} \psi & =\phi^{(0)} P_{2} \Psi\left(\sum_{i, j} \sum_{k} \lambda_{i j k} \sigma\left(e_{k}, f\right) e_{i} \otimes e_{j}\right) \Omega_{0} \\
& =\phi^{(0)} \sum_{i, j} \sum_{k} \lambda_{i j k} \sigma\left(e_{k}, f\right) P_{2}: \Phi\left(e_{i}\right) \Phi\left(e_{j}\right): \Omega_{0} \\
& =\frac{1}{2} \psi^{(0)} \sum_{i, j} \sum_{k} \lambda_{i j k} \sigma\left(e_{k}, f\right) a\left(\widetilde{e_{i}}\right) * a\left(\widetilde{e_{j}}\right) * \Omega_{0} \\
& =\frac{1}{2} \frac{\sqrt{2}}{2} \phi^{(0)} \sum_{i, j} \sum_{k} \lambda_{i j k} \sigma\left(e_{k}, f\right)\left(0,0, \widetilde{e_{i}} \otimes \widetilde{e_{j}}+\widetilde{e_{j}} \otimes \widetilde{e_{i}}, 0, \ldots\right) \\
& =\frac{1}{\sqrt{2}} \psi^{(0)}\left(0,0, \sum_{i, j} \sum_{k} \lambda_{i j k} \sigma\left(e_{k}, f\right) \widetilde{e_{i}} \otimes \widetilde{e_{j}}, 0,0, \ldots\right) .
\end{aligned}
$$

Since $\left\{\tilde{e}_{\imath}\right\}$ is an orthonormal basis in $\mathscr{H}$, one finds for the norm squared:

$$
\left\|P_{2} \Psi(\Lambda f) P_{0} \phi\right\|^{2}=\frac{1}{2}\left|\phi^{(0)}\right|^{2} \sum_{i, j}\left(\sum_{k} \lambda_{i j k} \sigma\left(e_{k}, f\right)\right)^{2}=\frac{1}{2}\left|\phi^{(0)}\right|^{2}\|\Lambda f\|^{2}
$$

Up to this point $\phi$ was an arbitrary vector from $U \mathscr{F}_{0}$, which is dense in Fock space. Thus choose $\phi$ such that $\left\|\phi-\Omega_{0}\right\|<\varepsilon$; then one has

$$
\varepsilon^{2}>\left\|\phi-\Omega_{0}\right\|^{2}=\left\|\left(\phi^{(0)}-1, \phi^{(1)}, \phi^{(2)}, \ldots\right)\right\|^{2}=\left|\phi^{(0)}-1\right|^{2}+\sum_{n=1}^{\infty}\left\|\phi^{(n)}\right\|^{2},
$$

which implies $\varepsilon>\left|1-\phi^{(0)}\right| \geq 1-\left|\phi^{(0)}\right|$, yielding $\left|\phi^{(0)}\right|>1-\varepsilon$. Thus one finds that for this choice of $\phi$, one has $\left\|P_{2} \Psi(\Lambda f) P_{0} \psi\right\| \geq \frac{1-\varepsilon}{\sqrt{2}}\|\Lambda f\|$.

Now to the second term in $(4.2 .1)$. In this case one has 


$$
\begin{aligned}
\left\|P_{2} \Psi(\Lambda f)\left(P_{2}+P_{4}\right) \phi\right\| & \leq\left\|\Psi(\Lambda f)\left(P_{2}+P_{4}\right) \phi\right\| \\
& \leq\left\|\Psi(\Lambda f)\left(P_{2}\right) \phi\right\|+\left\|\Psi(\Lambda f) P_{4} \phi\right\| \\
& \leq 2 \sqrt{2} \sqrt{3 \cdot 4}\|\Lambda f\|\left\|\phi^{(2)}\right\|+2 \sqrt{2} \sqrt{5 \cdot 6}\|\Lambda f\|\left\|\phi^{(4)}\right\|
\end{aligned}
$$

(see (3.1.1)). From (4.2.2) one extracts the bounds $\left\|\psi^{(2)}\right\|<\varepsilon$ and $\left\|\phi^{(4)}\right\|<\varepsilon$ for the specified choice of $\phi$, so that $\left\|P_{2} \Psi(\Lambda f)\left(P_{2}+P_{4}\right) \phi\right\| \leq 2 \sqrt{2}(\sqrt{3 \cdot 4}+\sqrt{5 \cdot 6}) \varepsilon\|\Lambda f\|$ $\leq 2 \sqrt{2}(\sqrt{4 \cdot 4}+\sqrt{6 \cdot 6}) \varepsilon\|\Lambda f\|=20 \sqrt{2} \varepsilon\|\Lambda f\|$.

With these two results, one can now continue (4.2.1):

$$
\left\|P_{2} \Psi(\Lambda f)\left(P_{0}+P_{2}+P_{4}\right) \psi\right\| \geq\left(\frac{1-\varepsilon}{\sqrt{2}}-20 \sqrt{2} \varepsilon\right)\|\Lambda f\|=\beta\|\Lambda f\|,
$$

with $\beta \equiv \frac{1-\varepsilon}{\sqrt{2}}-20 \sqrt{2} \varepsilon$. Choosing $\varepsilon<\frac{1}{41}$, one finds $\beta>0$. Therefore, for arbitrary $f \in D(\Lambda)$ one has found that

$$
\|\Lambda f\| \leq \frac{1}{\beta}\left\|P_{2} \Psi(\Lambda f)\left(P_{0}+P_{2}+P_{4}\right) \psi\right\| .
$$

Since it has already been demonstrated that the map $f \mapsto P_{2} \Psi(\Lambda f)\left(P_{0}+P_{2}+P_{4}\right) \psi$ is bounded, this implies that the operator $\Lambda$ is bounded, completing the proof.

This lemma is then used in the proof of the next proposition.

Proposition 4.2.2. Let $\Lambda \in \mathscr{L}_{C C R}$. If the canonical transformation $\Phi(f) \rightarrow$ $\Phi_{\Lambda}(f)$ is unitarily implementable, then $\Lambda$ is Hilbert-Schmidt.

Proof. 1. By Lemma 4.2.1 it may be assumed that $\Lambda$ is bounded and everywhere defined. Certainly $\Lambda_{\max }$ is bounded, so $\bar{\Lambda} \subset \Lambda_{\max }$ is everywhere defined. But it is straightforward to show that $U \Phi(f) U^{*}=\Phi_{\Lambda}(f)$, for all $f \in$ $D(\Lambda)$, entails $U \Phi(f) U^{*}=\Phi_{\bar{\Lambda}}(f)$, for all $f \in D(\bar{\Lambda})=H$, since it has already been remarked that $f_{n} \rightarrow f$ in $H$ implies that the self-adjoint $\Phi_{\Lambda}(f)$ is the strong graph limit of the self-adjoint $\Phi_{\Lambda}\left(f_{n}\right)$ (see [18], pp. 62-63 for further details).

It may therefore be assumed that $U \Phi(f) U^{*}=\Phi_{\Lambda}(f)$ for all $f \in H, \Lambda$ is bounded and $U$ is unitary. Choose a symplectic orthonormal basis $\left\{e_{k}, e_{k^{\prime}}\right\}$ in $H$, in which $\Lambda$ takes on its standard form, and consider

$$
\begin{aligned}
\Omega & \equiv U \Omega_{0}, \\
a_{k} & \equiv a\left(\widetilde{e_{k}}\right)=\frac{1}{\sqrt{2}}\left(\Phi\left(e_{k}\right)+i \Phi\left(J e_{k}\right)\right)=\frac{1}{\sqrt{2}}\left(q_{k}+i p_{k}\right),
\end{aligned}
$$




$$
U a_{k} U^{*}=\frac{1}{\sqrt{2}}\left(\Phi_{\Lambda}\left(e_{k}\right)+i \Phi_{\Lambda}\left(J e_{k}\right)\right)=\frac{1}{\sqrt{2}}\left(q_{k}+\overline{\left.i p_{k}+i \sum_{l, m} \lambda_{k l m}: q_{l} q_{m}:\left.\right|_{\mathscr{F}_{0}}\right)}\right.
$$

where (3.3.4) has been used. With $\Omega \equiv U \Omega_{0}$, one has $U a_{k} U^{*} \Omega=0$, so that (for $n \geq 2$ )

$$
\begin{aligned}
0 & =P_{n} U a_{k} U^{*} \Omega=P_{n} U a_{k} U^{*}\left(P_{n-2}+\cdots+P_{n+2}\right) \Omega \\
& =P_{n}\left(a_{k}+\frac{i}{2 \sqrt{2}} \sum_{l, m} \lambda_{k l m}\left(a_{l}^{*} a_{m}^{*}+2 a_{l}^{*} a_{m}+a_{l} a_{m}\right)\right)\left(P_{n-2}+\cdots+P_{n+2}\right) \Omega \\
& =a_{k} P_{n+1} \Omega+\frac{i}{2 \sqrt{2}} \sum_{l, m} \lambda_{k l m}\left(a_{l}^{*} a_{m}^{*} P_{n-2} \Omega+2 a_{l}^{*} a_{m} P_{n} \Omega+a_{l} a_{m} P_{n+2} \Omega\right) .
\end{aligned}
$$

Expanding with respect to the basis $\left\{\tilde{e}_{i}\right\}$, one writes $P_{m} \Omega=\left(0, \ldots, 0, \Omega^{(m)}, 0, \ldots\right)$ and $\Omega^{(m)}=\sum_{i_{1}, \ldots, i_{m}} \omega_{i_{1}, \ldots, i m}^{(m)} \tilde{e}_{i_{1}} \otimes \cdots \otimes \widetilde{e_{i m}}$, with $m \in \mathbb{N}$ and $\omega_{i_{1}, \ldots, i m}^{(m)} \in \mathbb{C}$, totally symmetric in the $m$ indices. Setting this into (4.2.3) and isolating a particular component $\widetilde{e_{i_{1}}} \otimes \cdots \otimes \widetilde{i_{i_{n}}}$, one finds for every $n \geq 2$,

$$
\begin{aligned}
& \sqrt{n+1} \omega_{k i_{1}, \ldots, i_{n}}^{(n+1)}+\frac{i}{2 \sqrt{2}}\left\{\frac{1}{\sqrt{n(n-1)}} \sum_{\substack{\nu, \mu=1 \\
\nu \neq \mu}}^{n} \lambda_{k i_{\nu} i_{\mu}} \omega_{i_{1} \ldots \hat{i} \ldots \ldots i_{\mu} \ldots i_{n}}^{(n-2)}\right. \\
& \left.+2 \sum_{m=1}^{\infty} \sum_{\nu=1}^{n} \lambda_{k m i_{\nu}} \omega_{m i_{1} \ldots \hat{i}_{\nu} \ldots i_{n}}^{(n)}+\sqrt{(n+2)(n+1)} \sum_{l, m=1}^{\infty} \lambda_{k l m} \omega_{l m i_{1} \ldots i_{n}}^{(n+2)}\right\}=0,
\end{aligned}
$$

where the symbol " over an index signifies that it should be left out of the sum.

2. For any $m \in \mathbf{N}$, one has $\left\|\Omega^{(m)}\right\|^{2}=\sum_{i_{1}, \ldots, i m}\left|\omega_{i_{1} \ldots i m}^{(m)}\right|^{2} \leq 1$ and, in particular, $\sum_{i_{1}, \ldots, i_{n}}\left|\omega_{k i_{1} \ldots i_{n}}^{(n+1}\right|^{2}<\infty$, for an arbitrary fixed index $k$. Moreover, since $\sum_{i_{\nu}, i_{\mu}} \lambda^{2}{ }_{k i_{\nu} i_{u}}$ $<\infty$ (see Prop. 3.3.1), one also has

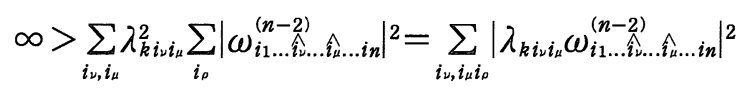

$$
\begin{aligned}
& =\sum_{i_{\nu}, i_{\nu} i_{\rho}}\left|\lambda_{k i_{\nu} i_{\rho}} \omega_{i_{1} \ldots \hat{i_{\nu} \ldots i_{\rho} \ldots i_{n}}}^{\left(n-\hat{i}_{2}\right)}\right|^{2} .
\end{aligned}
$$

To study the term $\sum_{m=1} \lambda_{k m i_{\nu}} \omega_{m i_{1} \ldots \hat{\nu} \ldots . . i_{n}}^{(n)}$ in $(4.2 .4)$ choose a vector $\sum_{m, i_{1}, \ldots, \hat{\nu}, \ldots, i_{n}} \Re e \omega_{m i_{1} \ldots \hat{i}_{u} \ldots i_{n}}^{(n)} \ell_{m} \otimes \ldots \otimes e_{i n}$ from $H^{\otimes n}$ (the $n$-fold direct product of $H$ with itself) and apply to it the operator $(\Lambda J) \otimes I^{\otimes n}$, which is continuous and defined everywhere. This results in a vector from $H^{\otimes n+1}$.

$$
\begin{aligned}
\left(\Lambda J \otimes I^{\otimes n}\right) & \sum_{m, i_{1}, \ldots, \hat{\nu}, \ldots, i_{n}} \\
& \Re e \omega_{m i_{1} \ldots \hat{i}_{\nu} \ldots i_{n}}^{(n)} e_{m} \otimes \cdots \otimes e_{i_{n}} \\
& =\sum_{m, i_{1}, \ldots, \hat{\hat{i}_{\nu}, \ldots, i_{n}}} \mathfrak{R} e \omega_{m i_{1} \ldots \hat{i}_{\nu} \ldots i_{n}}^{(n)}\left(\Lambda J e_{m}\right) \otimes \cdots \otimes e_{i_{n}}
\end{aligned}
$$




$$
=\sum_{m, i_{1}, \ldots, \hat{i}_{\nu}, \ldots, i_{n}} \Re e \omega_{m i_{1} \ldots \hat{i} \ldots \ldots i_{n}}^{(n)}\left(\sum_{k, i} \lambda_{k i m} e_{k} \otimes e_{i}\right) \otimes \cdots \otimes e_{i n}
$$

The square norm of this vector equals $\sum_{k, i, i_{1}, \ldots, \hat{\hat{i}_{1} \ldots, \ldots, i n}}\left(\sum_{m} \lambda_{k i m} \Re e \omega_{m i_{1} \ldots \hat{\mathrm{i}} \ldots . . i_{n}}^{(n)}\right)^{2}$ and must be finite. The same considerations employing the imaginary part of $\omega_{m i_{1} \ldots \hat{i}_{\nu} \ldots i_{n}}^{(n)}$ yield $\sum_{k, i, i_{1}, \ldots, \hat{i}_{\nu}, \ldots, i_{n}}\left(\sum_{m} \lambda_{k i m} \Im m \omega_{m i_{1} \ldots \hat{\nu}_{\nu} \ldots i_{n}}^{(n)}\right)^{2}<\infty$, and together they lead to

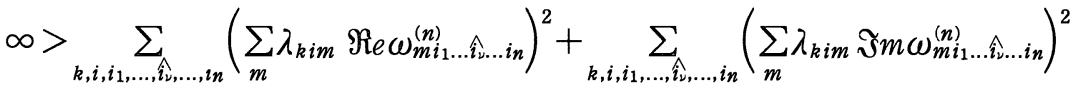

$$
\begin{aligned}
& =\sum_{k, i, i_{1}, \ldots, \hat{\hat{\nu}} \ldots \ldots, i_{n}}\left\{\left(\Re e \sum_{m} \lambda_{k i m} \omega_{m i_{1} \ldots \hat{i} \ldots \ldots i n}^{(n)}\right)^{2}+\left(\Im m \sum_{m} \lambda_{k i m} \omega_{m i_{1} \ldots \hat{i} \ldots . i_{n}}^{(n)}\right)^{2}\right\} \\
& =\sum_{k, i, i_{1}, \ldots, \hat{\hat{i}_{\nu}, \ldots, i_{n}}}\left|\sum_{m} \lambda_{k i m} \omega_{m i_{1} \ldots \hat{i}_{2} \ldots i_{n}}^{(n)}\right|^{2} .
\end{aligned}
$$

For the last term in $(4.2 .4)$ the operator $(\Lambda J)^{*}$ is useful. With $\Lambda J e_{k}=$ $\sum_{l, m} \lambda_{k l m} e_{l} \otimes e_{m}$, one has $(\Lambda J)^{*} e_{l} \otimes e_{m}=\sum_{k} \lambda_{k l m} e_{k}$, and since $\Lambda J: H \mapsto H \otimes H$ is bounded, its adjoint $(\Lambda J)^{*}: H \otimes H \mapsto H$ and $(\Lambda J)^{*} \otimes I^{\otimes n-1}:(H \otimes H) \otimes H^{\otimes n-1} \mapsto H^{\otimes n}$ are bounded (and everywhere defined). Thus one has

$$
\begin{gathered}
(\Lambda J)^{*} \otimes I^{\otimes n-1} \sum_{l, m, i, i_{1} \ldots i n-1} \Re e \omega_{l m i 1 \ldots i n}^{(n+2)} e_{l} \otimes e_{m} \otimes e_{i_{1}} \otimes \cdots \otimes e_{i_{n}} \\
=\sum_{l, m, i_{1}, \ldots, i_{n-1}} \Re e \omega_{l m i 1 \ldots i n}^{(n+2)}\left(\sum_{k} \lambda_{k l m} e_{k}\right) \otimes e_{i_{1}} \otimes \cdots \otimes e_{i n},
\end{gathered}
$$

with the square norm satisfying $\sum_{k, i_{1}, \ldots, i_{n}}\left(\sum_{l m} \lambda_{k l m} \Re e \omega_{l m i_{1} \ldots i_{n}}^{(n+2)}\right)^{2}<\infty$. With this and the corresponding bound for the imaginary part, one finds as above that

$$
\sum_{k, i_{1}, \ldots, i_{n}}\left|\sum_{l, m} \lambda_{k l m} \omega_{l m i 1 \ldots i n}^{(n+2)}\right|^{2}<\infty
$$

3. Pick $\nu, \mu \in\{1, \ldots, n\}$ with $\nu \neq \mu$, and write $i=i_{\nu}, j=i_{\mu}$ and relabel $\left\{i_{1}, \ldots\right.$, $\left.\hat{i}_{\nu} \ldots \hat{i}_{\mu \ldots} i_{n}\right\}$ by $\left\{i_{3}, \ldots, i_{n}\right\}$. One has seen that all the terms in (4.2.4) except possibly $\lambda_{k i j} \omega_{i_{3} . . i_{n}}^{(n-2)}$ are square summable over $k, i$ and $j$, so that all other quantities entering into (4.2.4) and indexed by $k, i$ and $j$ can be viewed as vectors in $\ell_{2} \otimes \ell_{2} \otimes \ell_{2}$. Therefore solving (4.2.4) for $\lambda_{k i j} \omega_{i 3 . . i n}^{(n-2)}$, it follows that $\left(\lambda_{k i j} \omega_{i 3 . . i n}^{(n-2)}\right)_{k, i, j=1,2, \ldots}$ must be an element of $\ell_{2} \otimes \ell_{2} \otimes \ell_{2}$, i.e.,

$$
\sum_{k, i, j}\left|\lambda_{k i j} \omega_{i 3 \ldots i n}^{(n-2)}\right|^{2}=\left|\omega_{i 3 \ldots i n}^{(n-2)}\right|^{2} \sum_{k, \imath, j} \lambda_{k i j}^{2}<\infty
$$

so that $\sum_{k, i, j} \lambda_{k i j}^{2}<\infty$ (one can always find a nonzero $\omega_{i 3 \ldots i n}^{(n-2)}$ for some $n$, since $\Omega$ $\neq 0)$. With this the proof is completed, since 


$$
\|\Lambda\|_{2}^{2}=\sum_{K}\left\|\Lambda e_{K}\right\|^{2}=\sum_{K, I, J}\left\langle e_{I} \otimes e_{J}, \Lambda e_{K}\right\rangle^{2}=\sum_{k, i, j} \lambda_{k i j}^{2}
$$

so that $\|\Lambda\|_{2}<\infty$.

\section{§4.3. $\Lambda$ Hilbert-Schmidt Implies Unitary Equivalence}

We next wish to prove the converse to Proposition 4.2.2, i.e. that $\Lambda \in \mathscr{L}_{C C R}$ Hilbert-Schmidt entails that the canonical transformation $\Phi(f) \mapsto \Phi_{\Lambda}(f)$ is unitarily implementable. The previously demonstrated standard form of the elements of $\mathscr{L}_{C C R}$ will be crucial here, since it will enable us to use $Q$-space techniques to facilitate the explicit construction of the desired unitary $U$. Once the quadratic transformation has been written in standard form (3.3.1), the unitary implementing the transformation should be (at least formally):

$$
U=\exp \left(-\frac{i}{3} \sum_{i, j, k} \lambda_{i j k}: \Phi\left(e_{i}\right) \Phi\left(e_{j}\right) \Phi\left(e_{k}\right):\right)
$$

as can be verified with the help of the Baker-Campbell-Hausdorff formula

$$
e^{A} B e^{-A}=B+[A, B]+\frac{1}{2 !}[A,[A, B]]+\frac{1}{3 !}[A,[A,[A, B]]]+\cdots
$$

However, proving that if $\Lambda$ is Hilbert-Schmidt, then (4.3.1) actually defines a unitary operator intertwining the two representations is not trivial.

Let $\Lambda \in \mathscr{L}_{C C R}$ be given and then written in standard form, with $\left\{\lambda_{k l m}\right\}_{k, l, m \in \mathbf{N}}$ the resulting sequence of real numbers totally symmetric with respect to permutations of the indices $k, l, m$, and $\sum_{k, l, m=1}^{\infty} \lambda_{k l m}^{2}<\infty$, and let $\left\{e_{k}, e_{k}^{\prime}\right\}=\left\{e_{K} \mid K=1,1^{\prime}\right.$, $\left.2,2^{\prime}, \ldots\right\} \subset H$ be the resulting symplectic orthonormal basis. Moreover, let $x=$ $\left(x_{1}, x_{2}, \ldots\right)$ be a point in $Q \equiv \times_{k=1}^{\infty} \mathbb{R}$, and $\sum$ be the $\sigma$-algebra generated by the cylinder sets of $Q$ with Lebesgue measurable base. Then $\mu=\otimes_{k=1}^{\infty} \mu_{k}$, where

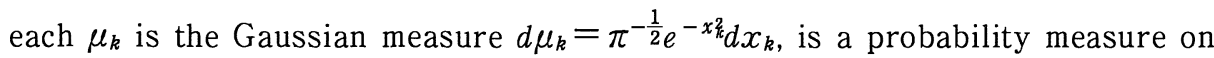
$(Q, \Sigma)$.

It is well-known that there exists a unitary map $S$ of $\mathscr{F}_{+}(\mathscr{H})$ onto $L^{2}(Q$, $d \mu)$ such that [24] [27] [21]

$$
\begin{aligned}
& S \Omega_{0}=1 \text { and } S P_{+}\left({\widetilde{e_{k}}}_{1} \otimes{\widetilde{e_{k 2}}} \otimes \cdots \otimes \widetilde{e_{k r}}\right)=(r !)^{-\frac{1}{2}}(\sqrt{2})^{r}: x_{k 1} x_{k 2} \cdots x_{k r}: \\
& S \Phi\left(e_{k}\right) S^{-1} \equiv q_{k}=\text { (multiplication by) } x_{k}, \text { and }
\end{aligned}
$$




$$
S \Phi\left(e_{k}\right) S^{-1} \equiv p_{k}=\frac{1}{i} \frac{\partial}{\partial x_{k}}+i x_{k}
$$

where the operator equations are understood to hold on the dense set $S \mathscr{F}_{0}$. We shall occasionally drop the symbol $S$, when the identification is clear.

Let $\underline{k}=\left\{k_{1}, k_{2}, \ldots, k_{r}\right\}$ be a multiple index in $\mathbf{N}^{(r)}=\times_{j=1}^{r} \mathbf{N},\left\{\lambda_{\underline{k}}\right\}_{\underline{k} \in \mathbf{N}^{(r)}}$ a sequence of real numbers, totally symmetric in $\underline{k}, \sum_{\underline{k}} \lambda_{\underline{k}}^{2}<\infty$, and $I_{n}=\left\{\underline{k} \mid \max \left\{k_{1}, k_{2}\right.\right.$, $\left.\left.\ldots, k_{r}\right\} \leq n\right\}$. Then

$$
f_{n}^{(r)}=\sum_{\underline{k} \in I_{n}} \lambda_{\underline{\underline{k}}} e_{k_{1}} \otimes_{e_{k_{2}}} \otimes \cdots \otimes \otimes_{e_{r}} \in H^{(r)},
$$

with $\left\|f_{n}^{(r)}\right\|^{2}=\sum_{\underline{\underline{k}} \in I_{n}} \lambda_{\underline{\underline{k}}}^{2}$. Since with $m<n$, we have $\left\|f_{n}^{(r)}-f_{m}^{(r)}\right\|^{2}=\sum_{\underline{\underline{k}} \in I_{n} \backslash I_{m}} \lambda_{\underline{k}}^{2}$ and $\sum_{\underline{k} \in I_{n}} \lambda_{\underline{k}}^{2}$ converges with $n \rightarrow \infty$, it follows that

$$
f_{n}^{(r)} \rightarrow f^{(r)}=\sum_{\underline{k}} \lambda_{\underline{k}} e_{k_{1}} \otimes e_{k_{2}} \otimes \cdots \otimes e_{k r} \text { in } H^{(r)}
$$

We want to study the sequence of operators

$$
A\left(f_{n}^{(r)}\right)=\sum_{\underline{k} \in I_{n}} \lambda_{\underline{k}}: \Phi\left(e_{k_{1}}\right) \Phi\left(e_{k_{2}}\right) \cdots \Phi\left(e_{k_{r}}\right):
$$

the $Q$-space realization of which is given by $A_{n}=A\left(f_{n}^{(r)}\right)=\sum_{\underline{k} \in I_{n}} \lambda_{\underline{k}}: x_{k_{1}} x_{k_{2}} \cdots x_{k_{r}}:$. Note that since it is a polynomial, $A_{n}=A_{n}(x)$ is in $L^{2}(Q, d \mu)$ with

$$
\begin{gathered}
\left\|A_{n}\right\|^{2}=2^{-r} r ! \sum_{\underline{k} \in I n} \lambda_{\underline{\underline{k}}}^{2}=2^{-r} r !\left\|f_{n}^{(r)}\right\|^{2}, \\
\text { and }\left\|A_{n}-A_{m}\right\|^{2}=2^{-r} r ! \quad\left\|f_{n}^{(r)}-f_{m}^{(r)}\right\|^{2}
\end{gathered}
$$

(cf. the proof of Lemma I.18 of [27]). Therefore, $A_{n}(x)$ converges in $L^{2}(Q, d \mu)$, and we shall call the a.e.-defined limit $A(x)=\sum_{\underline{k}} \lambda_{\underline{k}}: x_{k_{1}} x_{k_{2}} \cdots x_{k_{r}}:$, which up to a factor of $\sqrt{r !}(\sqrt{2})^{-r}$ corresponds to $f^{(r)}$.

The advantage of the $Q$-space formulation is that all functions of $\left\{\Phi\left(e_{k}\right), k\right.$ $=1,2, \ldots\}$ become multiplication operators on $L^{2}(Q, d \mu) . A_{n}(x)$ and $A(x)$ are measurable, real-valued functions on $Q$ which are finite a.e. $[\mu]$. So with $D(A)$ $\equiv\left\{\varphi \mid A \varphi \in L^{2}(Q, d \mu)\right\}$ (similarly for $\left.D\left(A_{n}\right)\right),\left(A_{n} \varphi\right)(x)=A_{n}(x) \varphi(x)$ and $(A \varphi)(x)$ $=A(x) \varphi(x)$ are self-adjoint operators (cf. [20], VIII. 3 Proposition 1). Thus, for every $f^{(r)} \in P_{+} H^{(r)}, A\left(f^{(r)}\right)$ represents a well-defined self-adjoint multiplication operator.

We collect a number of useful technical results concerning these operators. 
Lemma 4.3.1. 1. $L^{\infty}(Q, d \mu) \subset L^{2}(Q, d \mu)$ is a common core for all $A_{n}, A$.

2. As operators on $L^{2}(Q, d \mu), A_{n} \rightarrow A$ in the strong resolvent sense.

3. On $L^{\infty}(Q, d \mu)$ the operator family $A\left(f^{(r)}\right)$ is strongly continuous in $f^{(r)} \in$ $P_{+} H^{(r)}$.

4. Let $f$ be a bounded continuous function on $\mathbb{R}$. Then $f\left(A_{n}\right) \varphi \rightarrow f(A) \varphi$ for all $\varphi \in L^{2}(Q, d \mu)$, and especially, $e^{i t A n} e^{i t A}$ strongly for each $t \in \mathbb{R}$. Moreover, $A$ is the strong graph limit of $A_{n}$.

Proof. 1. Of course, $L^{\infty}(Q, d \mu)$ is dense in $L^{2}(Q, d \mu)$. For any vector $\varphi \in$ $L^{\infty}(Q, d \mu)$, one has $\left\|A_{n} \varphi\right\| \leq\|\varphi\|_{\infty}\left\|A_{n}\right\|<\infty$ and $\|A \varphi\| \leq\|\varphi\|_{\infty}\|A\|<\infty$, showing that $L^{\infty}(Q, d \mu) \subset D\left(A_{n}\right)$ and $L^{\infty}(Q, d \mu) \subset D(A)$, respectively. $A_{n}, A$ are multiplication by real-valued functions. Hence, for all $t \in \mathbf{R}$, one has $e^{i t A n}: L^{\infty}(Q, d \mu) \rightarrow L^{\infty}(Q$, $d \mu)$ and $e^{i t A}: L^{\infty}(Q, d \mu) \rightarrow L^{\infty}(Q, d \mu)$. Assertion (1) now follows from [20], Theorem VIII.11.

2. For $\varphi \in L^{\infty}(Q, d \mu)$ one has $\left\|A_{n} \varphi-A \varphi\right\| \leq\|\varphi\|_{\infty}\left\|A_{n}-A\right\| \underset{n \rightarrow \infty}{\rightarrow 0}$. Hence, $A_{n} \varphi \rightarrow A \varphi$ for each $\varphi \in L^{\infty}(Q, d \mu)$, which according to [20], Theorem VIII.25, implies $A_{n} \rightarrow A$ in the strong resolvent sense.

3. For $\varphi \in L^{\infty}(Q, d \mu)$ one has from Step (2) and equation (4.3.2)

$$
\left\|A\left(f^{(r)}\right) \varphi-A\left(g^{(r)}\right) \varphi\right\|^{2} \leq 2^{-r} r !\|\varphi\|_{\infty}^{2}\left\|f^{(r)}-g^{(r)}\right\|^{2}
$$

4. Assertion (4) follows from the previous steps and standard theorems (see, e.g. [20], Theorems VIII.20, VIII.21 and VIII.26).

Remark. Assertion 4 means in particular that if $f_{n}=\sum_{k=1}^{n} \alpha_{k} e_{k} \rightarrow f \in H$ then $\Phi(f)$ is the strong graph limit of $\Phi\left(f_{n}\right)$. Since the role of $p_{k}$ and $q_{k}$ as multiplication operators could have been interchanged, it is also true that if $g_{n}=$ $\sum_{k=1}^{n} \beta_{k} J e_{k} \rightarrow g \in H$ then $\Phi(g)$ is the strong graph limit of $\Phi\left(g_{n}\right)$.

In the next lemma we want to show that $\mathscr{F}_{0} \subset D\left(A\left(f^{(r)}\right)\right.$ for all $f^{(r)} \in H^{(r)}$, that in contrast to $L^{\infty}(Q, d \mu), \mathscr{F}_{0}$ is invariant under $A\left(f^{(r)}\right)$ and that the analogue of Lemma 4.3.1, Assertion 3, also holds on $\mathscr{F}_{0}$. Note that on $\mathscr{F}_{0}$, we have $\Phi\left(e_{k}\right)=\frac{1}{\sqrt{2}}\left(a^{*}\left(\widetilde{e_{k}}\right)+a\left(\widetilde{e_{k}}\right)\right)$. Inserting $\frac{1}{\sqrt{2}}\left(a^{*}\left(\widetilde{e_{k}}\right)+a\left(\widetilde{e_{k}}\right)\right)$ for each $\Phi\left(e_{k}\right)$ in $A$ $\left(f_{n}^{(r)}\right)=\sum_{\underline{k} \in I n} \lambda_{\underline{k}}: \Phi\left(e_{k_{1}}\right) \Phi\left(e_{k_{2}}\right) \cdots \Phi\left(e_{k_{r}}\right):$, we observe that $A\left(f_{n}^{(r)}\right)$ is a finite sum of finite products of $a^{*}\left(\widetilde{e}_{k}\right), a\left(\widetilde{e}_{k}\right)$ under which $\mathscr{F}_{0}$ is invariant. Hence, $\mathscr{F}_{0} \subset D$ $\left(A_{n}\right)$ for all $n$.

In order to express $A\left(f_{n}^{(r)}\right)$ in terms of $a^{*}\left(\widetilde{e_{k}}\right), a\left(\widetilde{e_{k}}\right)$ we define for $0 \leq j \leq r$ 


$$
B_{j}\left(f_{n}^{(r)}\right)=\sum_{\underline{k} \in I n} \lambda_{\underline{k}} a^{*}\left({\widetilde{e_{k},+1}}\right) a^{*}\left({\widetilde{e_{k},+2}}\right) \cdots a^{*}\left(\widetilde{e_{k r}}\right) a\left(\widetilde{e_{k}}\right) a\left(\widetilde{e_{k}-1}\right) \cdots a\left({\widetilde{e_{k 1}}}\right)
$$

Since with $\underline{k} \in I_{n}$ we sum over a symmetric index set and since $\lambda_{\underline{k}}$ is totally symmetric in $\underline{k}, B_{j}\left(f_{n}^{(r)}\right)$ does not depend on the specific ordering of the indices $\left\{k_{1}\right.$, $\left.k_{2}, \ldots, k_{r}\right\}$. Taking into account this symmetry we see that on $\mathscr{F}_{0}$ the equality

$$
A\left(f_{n}^{(r)}\right)=(\sqrt{2})^{-r} \sum_{j=0}^{r}\left(\begin{array}{l}
r \\
j
\end{array}\right) B_{j}\left(f_{n}^{(r)}\right)
$$

holds. Let us denote by $H_{s, 0}^{(r)}$ the subset of $P_{+} H^{(r)}$ which consists of finite symmetrized linear combinations of the basis $e_{k_{1}} \otimes e_{k_{2}} \otimes \cdots \otimes e_{k_{r}}$. Hence, $f_{n}^{(r)}$ is a typical example of a vector in $H_{s, 0}^{(r)}$.

Lemma 4.3.2. Let $f^{(r)} \in P_{+} H^{(r)}$. Then

1. $\mathscr{F}_{0} \subset D\left(A\left(f^{(r)}\right)\right)$,

2. $\mathscr{F}_{0}$ is invariant under $A\left(f^{(r)}\right)$,

3. $A\left(f^{(r)}\right)$ is strongly continuous in $f^{(r)}$ on $\mathscr{F}_{0}$.

Proof. By the preceding discussion, assertions (1) and (2) are obvious for $f^{(r)} \in H_{s, 0}^{(r)}$. Let $\varphi=\left\{\varphi^{(0)}, \varphi^{(1)}, \varphi^{(2)}, \ldots\right\} \in \mathscr{F}_{0}$ and $\varphi^{(s)}=0$ for $s>n_{\varphi}$. By a natural embedding $\varphi^{(s)}=\left\{0, \ldots, 0, \varphi^{(s)}, 0, \ldots\right\}$. Consider the expansion $\varphi^{(s)}=\sum_{\underline{i}} \varphi_{\underline{i}}^{(s)} \widetilde{e_{i_{1}}} \otimes \widetilde{e_{i_{2}}}$ $\otimes \cdots \otimes \widetilde{e_{i s}}$ with $\varphi_{\underline{i}}^{(s)} \in \mathbb{C}$, totally symmetric in $\underline{i}=\left\{i_{1}, \ldots, i_{s}\right\}$. Then, for $j \leq s \leq n_{\varphi}$,

$$
\begin{aligned}
& B_{j}\left(f_{n}^{(r)}\right) \varphi^{(s)} \\
& =P+\sum_{\underline{k} \in I_{n}} \sum_{i} \lambda_{\underline{k}} \varphi_{\underline{i}} n(s, j, r) \delta_{k 1 i_{1}} \delta_{k 2 i 2} \cdots \delta_{k, i}{\widetilde{e_{k j+1}}} \otimes \cdots \otimes \widetilde{e_{k r}} \otimes \widetilde{e_{i j+1}} \otimes \cdots \otimes \widetilde{e_{i s}} \\
& =P+\sum_{\underline{k} \in I_{n} i_{j+1} \ldots i_{s}} \lambda_{\underline{k}} \varphi_{k_{1} \ldots k_{j} i_{j+1} \ldots i_{s}} n(s, j, r) \widetilde{e_{k_{j+1}}} \otimes \cdots \otimes \widetilde{e_{k r}} \otimes \widetilde{e_{i_{j+1}}} \otimes \cdots \otimes \widetilde{e_{i_{s}}},
\end{aligned}
$$

where $n(s, j, r)=\sqrt{s(s-1) \cdots(s-j+1)} \sqrt{(s-j+1)(s-j+2) \cdots(s-j+r-j)}$. Taking into account that $P_{+}$is a projection operator, using the Schwarz inequality and $|n(s, j, r)|^{2} \leq s^{j}(s+r)^{r-j} \leq(s+r)^{r}$, one gets

$$
\left\|B_{j}\left(f_{n}^{(r)}\right) \varphi^{(s)}\right\|^{2} \leq(s+r)^{r}\left\|f_{n}^{(r)}\right\|^{2}\left\|\varphi^{(s)}\right\|^{2}
$$

Note that $B_{j}\left(f_{n}^{(r)}\right) \varphi^{(s)}=0$ for $j>s$, so the inequality (4.3.4) holds also in this case. Since $s \leq n_{\varphi}, \varphi=\sum_{s \underline{\underline{\varphi}}_{0}}^{n^{(s)}}$ and $\left\|\varphi^{(s)}\right\|^{2} \leq\|\varphi\|^{2},\left\|B_{j}\left(f_{n}^{(r)}\right) \varphi\right\| \leq\left(n_{\varphi}+1\right)\left(n_{\varphi}+\right.$ r) $\frac{r}{2}\left\|f_{n}^{(r)}\right\|\|\varphi\|$ by the triangle inequality. Inserting $A\left(f_{n}^{(r)}\right)$ in the form given by equation $(4.3 .3)$ we arrive at 


$$
\left\|A\left(f_{n}^{(r)}\right) \varphi\right\| \leq(\sqrt{2})^{r}\left(n_{\varphi}+r\right)^{\frac{r}{2}+1}\left\|f_{n}^{(r)}\right\|\|\varphi\| .
$$

Consequently, $\left\|A\left(g^{(r)}\right) \varphi-A\left(^{(r)}\right) \varphi\right\|=\left\|A\left(g^{(r)}-h^{(r)}\right) \varphi\right\| \leq(\sqrt{2})^{r}\left(n_{\varphi}+r\right)^{\frac{r}{2}+1} \| g^{(r)}-$ $h^{(r)}\|\| \varphi \|$ for all $g^{(r)}, h^{(r)} \in H_{s, 0}^{(r)}$, proving assertion (3) for $f^{(r)} \in H_{s, 0}^{(r)}$. Choosing $f_{n}^{(r)}$, $f_{m}^{(r)}$ for $g^{(r)}, h^{(r)}$ in (4.3.5), one sees that $A\left(f_{n}^{(r)}\right) \varphi$ converges, and, since $A\left(f^{(r)}\right)$ is the strong graph limit of $A\left(f_{n}^{(r)}\right), \varphi \in D\left(A\left(f^{(r)}\right)\right)$ and $A\left(f_{n}^{(r)}\right) \varphi \rightarrow A\left(f^{(r)}\right) \varphi$. This proves assertion (1) and together with (4.3.5) also assertion (3). Note that for all $n, A\left(f_{n}^{(r)}\right)$ creates at most $r$ particles. Thus $\left(A\left(f_{n}^{(r)}\right) \varphi\right)^{(s)}=0$ for $s>$ $n_{\varphi}+r$, which is then true also for the limit $A\left(f^{(r)}\right) \varphi$, i.e. $A\left(f^{(r)}\right) \varphi \in \mathscr{F}_{0}$.

We now come back to the standard form of $\Lambda$ and decompose $f \in H$ as $f^{r e}+$ $f^{i m}$, $f^{r e}=\sum_{k} \alpha_{k} e_{k}, f^{i m}=\sum_{k} \beta_{k} J e_{k}$, so that $\Lambda f=\sum_{l, m} \sum_{k} \lambda_{k l m} \beta_{k} e_{l} \otimes e_{m}$. Note that $\|\Lambda f\|^{2}$ $=\sum_{l, m}\left(\sum_{k} \lambda_{k l m} \beta_{k}\right)^{2} \leq \sum_{k, l, m} \lambda_{k l m}^{2}\|f\|^{2}<\infty$ showing $\Lambda f \in P_{+} H^{(2)}$ for all $f \in H$.

In Proposition 3.1.3 above it has been shown that $\Phi_{\Lambda}(f)=\Phi(f)+\Phi(\Lambda f)$ is essentially self-adjoint on $\mathscr{F}_{0}$. On $\mathscr{F}_{0}$ we have $\Phi_{\Lambda}\left(f^{r e}+f^{i m}\right)=\Phi_{\Lambda}\left(f^{r e}\right)+$ $\Phi_{\Lambda}\left(f^{i m}\right)=\Phi\left(f^{r e}\right)+\Phi\left(f^{i m}\right)+\Psi\left(\Lambda f^{i m}\right)$ with $\Psi\left(\Lambda f^{i m}\right)=\sum_{l, m} \sum_{k} \lambda_{k l m} \beta_{k}: \Phi\left(e_{l}\right) \Phi\left(e_{m}\right)$ :. It shall be shown that on $\mathscr{F}_{0}$ which is a core for $\Phi_{\Lambda}(f)$, one has

$$
\begin{gathered}
\Phi_{\Lambda}\left(f^{r e}\right)=\Phi\left(f^{r e}\right)=U \Phi\left(f^{r e}\right) U^{*} \text { and } \\
\Phi_{\Lambda}\left(f^{i m}\right)=\Phi\left(f^{i m}\right)+\Psi\left(\Lambda f^{i m}\right)=U \Phi\left(f^{i m}\right) U^{*}
\end{gathered}
$$

with the unitary operator $U=e^{-\frac{i}{3} A\left(f^{(3)}\right)}$, where $f^{(3)}=\sum_{k, l, m} \lambda_{k l m e_{k}} \otimes e_{l} \otimes e_{m}$ and $A\left(f^{(3)}\right)=\sum_{k, l, m} \lambda_{k l m}: \Phi\left(e_{k}\right) \Phi\left(e_{l}\right) \Phi\left(e_{m}\right):$.

In $L^{2}(Q, d \mu), A\left(f^{(3)}\right)$ is multiplication by $A(x)=\sum_{k, l, m} \lambda_{k l m}: x_{k} x_{l} x_{m}:$ and $U^{*}$ is multiplication by $e^{\frac{i}{3} A(x)}$. Similarly, $\Phi\left(f^{r e}\right)$, is multiplication by $\Phi_{f r e}(x)=$ $\sum_{k} \alpha_{k} x_{k}$ with $D\left(\Phi\left(f^{r e}\right)\right)=\left\{\varphi \mid \Phi\left(f^{r e}\right) \varphi \in L^{2}(Q, d \mu)\right\}$. Therefore, $D\left(\Phi\left(f^{r e}\right)\right)$ is left invariant by $U^{*}$, and, obviously, $U \Phi\left(f^{r e}\right) U^{*} \varphi=\Phi\left(f^{r e}\right) \varphi$ for all $\varphi \in D\left(\Phi\left(f^{r e}\right)\right) \supset$ $\mathscr{F}_{0}$.

Next consider $\Phi_{\Lambda}\left(J e_{i}\right)=\Phi\left(e_{i}\right)+\Psi\left(\Lambda J e_{i}\right)=\Phi\left(J_{i}\right)+\sum_{l, m} \lambda_{i l m}: \Phi\left(e_{l}\right) \Phi\left(e_{m}\right):$, which on $S \mathscr{F}_{0} \subset L^{2}(Q, d \mu)$ takes the form

$$
\Phi_{\Lambda}\left(J e_{i}\right)=p_{i}+\sum_{l, m} \lambda_{i l m}: x_{l} x_{m}: \quad \text { with } \quad p_{i}=\frac{1}{i} \frac{\partial}{\partial x_{i}}+i x_{i} .
$$

Lemma 4.3.3. $A(x)=\sum_{k, l, m} \lambda_{k l m}: x_{k} x_{l} x_{m}:$ is differentiable by every $x_{i}$ and $\frac{\partial A}{\partial x_{i}}(x)=3 \sum_{l, m} \lambda_{i l m}: x_{l} x_{m}:$ 
Proof. Writing $A(x)$ in the form

$$
\begin{aligned}
A(x) & =\lambda_{i n l}: x_{i}^{3}:+3 \sum_{l \neq i} \lambda_{i i l}: x_{l}^{2} x_{l}:+3 \sum_{l, m \neq i} \lambda_{i l m}: x_{i} x_{l} x_{m}:+\sum_{k, l, m \neq i} \lambda_{k l m}: x_{k} x_{l} x_{m}: \\
& =\lambda_{\text {iii }}: x_{i}^{3}:+3: x_{i}^{2}: \sum_{l \neq i} \lambda_{i i l}: x_{l}:+3: x_{\imath}: \sum_{l, m \neq i} \lambda_{i l m}: x_{l} x_{m}:+\sum_{k, l, m \neq i} \lambda_{k l m}: x_{k} x_{l} x_{m}:
\end{aligned}
$$

one sees that

$$
\begin{aligned}
\frac{\partial A}{\partial x_{i}}(x) & =3\left(\lambda_{i t i}: x_{i} x_{i}:+2 \sum_{l \neq i} \lambda_{i i l}: x_{i} x_{l}:+\sum_{l, m \neq i} \lambda_{i l m}: x_{l} x_{m}:\right) \\
& =3 \sum_{l, m} \lambda_{i l m}: x_{l} x_{m}:
\end{aligned}
$$

The convergence of the respective infinite sums is the same as discussed following (4.3.2).

Since $\sum_{l, m} \lambda_{i l m e_{l}} \otimes_{e_{m}} \in P_{+} H^{(2)}$, we see by Lemma 4.3 .2 that $U^{*} \mathscr{F}_{0} \subset D\left(p_{i}\right)$, because for $\varphi \in \mathscr{F}_{0}, p_{i} U^{*} \varphi=\left(\frac{1}{i} \frac{\partial}{\partial x_{i}}+i x_{i}\right) e^{\frac{i}{3} A(x)} \varphi(x)=e^{\frac{i}{3} A(x)}\left(\left(\frac{1}{i} \frac{\partial}{\partial x_{i}}+i x_{i}\right)+\right.$ $\left.\frac{1}{3} \frac{\partial A}{\partial x_{i}}(x)\right) \varphi(x)$. Hence

$$
p_{i} U^{*} \varphi=\left(p_{i}+\sum_{l, m} \lambda_{\imath l m}: x_{l} x_{m}:\right) \varphi
$$

i.e. $U \Phi\left(J e_{\imath}\right) U^{*} \varphi=\Phi_{\Lambda}\left(J e_{i}\right) \varphi$ for all $\varphi \in \mathscr{F}_{0}$. Equation (4.3.6) also implies $U \Phi\left(f^{i m}\right) U^{*}=\Phi_{\Lambda}\left(f^{i m}\right)$ on $\mathscr{F}_{0}$ for those $f^{i m}$ which are finite linear combinations of $\left\{J e_{k}\right\}_{k=1}^{\infty}$. Now let $f_{n}^{i m}=\sum_{k=1}^{n} \beta_{k} J e_{k} \rightarrow f_{n \rightarrow \infty}^{i m} \in H$. Then, with $\varphi \in \mathscr{F}_{0}$,

$$
\begin{aligned}
\Phi\left(f_{n}^{i m}\right) U^{*} \varphi & =U^{*} \Phi_{\Lambda}\left(f_{n}^{i m}\right) \varphi=U^{*}\left(\Phi\left(f_{n}^{i m}\right)+\sum_{l, m} \sum_{k} \lambda_{k l m} \beta_{k}: \Phi\left(e_{l}\right) \Phi\left(e_{m}\right):\right) \varphi \\
& =U^{*}\left(\Phi\left(f_{n}^{i m}\right) \varphi+A\left(\Lambda f_{n}^{i m}\right) \varphi\right) .
\end{aligned}
$$

Since $\Lambda$ is bounded, $\Lambda f_{n}^{i m} \rightarrow \Lambda f^{i m} \in P_{+} H^{(2)}$ and by Lemma 4.3.2, $A\left(\Lambda f_{n}^{i m}\right) \varphi \rightarrow$ $A\left(\Lambda f_{n}^{i m}\right) \varphi$. Taking limits on both sides of equation (4.3.7) yields, according to

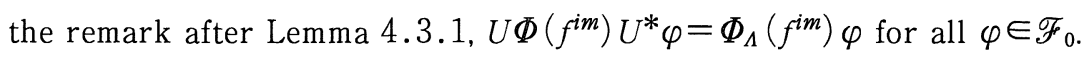

We summarize the results of this section as

Proposition 4.3.4. Let $\Phi(f) \mapsto \Phi_{\Lambda}(f)$ be a quadratic transformation of the CCR. If $\Lambda \in \mathscr{L}_{C C R}$ is Hilbert-Schmidt, then $\Phi_{\Lambda}(f)$ is unitarily equivalent to the Fock representation $\Phi(f)$.

It is clear then that Theorem 4.1 is a consequence of Theorem 4.1.1, Corollary 
4.1.2, and Propositions 4.2.2 and 4.3.4.

\section{Additional Comments and Developments}

In this chapter we collect a number of relevant comments on further developments and possible applications of quadratic transformations.

\section{Heuristic Comments on General Quadratic Canonical Transformations}

Combining the purely quadratic transformations we have rigorously studied above with the well-known inhomogeneous linear transformations of [1], we obtain the general (formal) quadratic transformation of $\Phi: \Phi(f) \mapsto \Phi_{\text {gen }}(f)$, where

$$
\begin{aligned}
\Phi_{\text {gen }}(f) \equiv \sum_{k=1}^{\infty} & \left(\alpha_{k}(f) a_{k}^{*}+\widetilde{\alpha}_{k}(f) a_{k}\right) \\
& \quad+\sum_{k, l=1}^{\infty}\left(\alpha_{k l}(f) a_{k}^{*} a_{l}^{*}+\widetilde{\alpha}_{k l}(f) a_{k} a_{l}+2 \beta_{k l}(f) a_{k}^{*} a_{l}\right)+\ell(f),(5.1)
\end{aligned}
$$

with $f \in H, \alpha_{k}, \widetilde{\alpha}_{k}, \alpha_{k l}, \widetilde{\alpha}_{k l}, \beta_{k l}, \ell$ (complex-valued) linear functionals on $H$ and $a_{k}=a\left(\widetilde{e_{k}}\right), a_{k}^{*}=a\left(\widetilde{e_{k}}\right)^{*}$ annihilator and creator for the $k$-th degree of freedom $\left(\left\{\widetilde{e_{k}}\right\}\right.$ is an orthonormal basis in $\left.\mathscr{H}\right)$. A term of the form $a_{k} a_{l}^{*}$ can be absorbed into the $a_{k}^{*} a_{l}$-and $\ell$-terms using the commutation relations. $\Phi_{\text {gen }}(f)$ should be symmetric, whence it follows that $\widetilde{\alpha}_{k}(f)=\overline{\alpha_{k}(f)}, \widetilde{\alpha}_{k l}(f)=\overline{\alpha_{k l}(f)}$, and $\beta_{k l}(f)=$ $\overline{\beta_{l k}(f)}, \ell(f) \in \mathbb{R}$. Since $a_{k}^{*}$ commutes with $a_{l}^{*}$, one may assume without loss of generality that $\alpha_{k l}(f)=\alpha_{l k}(f)$.

The 'linear' part of $\Phi_{\text {gen }}(f)$ can be written as

$$
\sum_{k=1}^{\infty}\left(\alpha_{k}(f) a_{k}^{*}+\overline{\alpha_{k}(f)} a_{k}\right)=\Phi(T f)
$$

with $T: H \mapsto H$ defined by

$$
T f \equiv \sqrt{2} \sum_{k=1}^{\infty}\left\{\Re e \alpha_{k}(f) e_{k}+\Im m \alpha_{k}(f) e_{k^{\prime}}\right\}
$$

or equivalently $\widetilde{T f}=\sqrt{2} \sum_{k} \alpha_{k}(f) \widetilde{e}_{k}$. Defining, in addition, the operator $\Gamma: H \mapsto$ $P_{+}(H \otimes H)$ by

$$
\Gamma f=\sum_{K, L} \gamma_{K L}(f) e_{K} \otimes e_{L}, \quad \gamma_{K L}(f) \in \mathbb{R}, \quad \gamma_{K L}(f)=\gamma_{L K}(f),
$$


with $\gamma_{k l}(f) \equiv \Re e\left(\alpha_{k l}(f)+\beta_{k l}(f)\right), \gamma_{k^{\prime} l^{\prime}}(f) \equiv \Re e\left(-\alpha_{k l}(f)+\beta_{k l}(f)\right), \gamma_{k l^{\prime}}(f) \equiv$ $\Im m\left(\alpha_{k l}(f)-\beta_{k l}(f)\right)$, and $\gamma_{k^{\prime} l}(f) \equiv \Im m\left(\alpha_{k l}(f)+\beta_{k l}(f)\right)$ and setting it into the two-particle operator $\Psi(\cdot)$, we obtain

$$
\begin{gathered}
\Psi(\Gamma f)=\frac{1}{2} \sum_{K, L} \gamma_{K L}(f)\left\{a\left(\widetilde{e_{K}}\right) * a\left(\widetilde{e_{L}}\right) *+a\left(\widetilde{e_{K}}\right) a\left(\widetilde{e_{L}}\right)+2 a\left(\widetilde{e_{K}}\right) * a\left(\widetilde{e_{L}}\right)\right\} \\
=\frac{1}{2} \sum_{k, l}\left\{\left(\gamma_{k l}(f)-\gamma_{k^{\prime} l^{\prime}}(f)+i \gamma_{k l^{\prime}}(f)+i \gamma_{k^{\prime} l}(f)\right) a_{k}^{*} a_{l}^{*}\right. \\
+\left(\gamma_{k l}(f)-\gamma_{k^{\prime} l^{\prime}}(f)-i \gamma_{k l^{\prime}}(f)-i \gamma_{k^{\prime} l}(f)\right) a_{k} a_{l} \\
\left.+2\left(\gamma_{k l}(f)+\gamma_{k^{\prime} l^{\prime}}(f)-i \gamma_{k l^{\prime}}(f)+i \gamma_{k^{\prime} l}(f)\right) a_{k}^{*} a_{l}\right\},
\end{gathered}
$$

which is exactly the quadratic part of $\Phi_{\text {gen }}(f)$, since $\gamma_{k l}(f)-\gamma_{k^{\prime} l^{\prime}}(f)+i \gamma_{k l^{\prime}}(f)$ $+i \gamma_{k^{\prime} l}(f)=2 \alpha_{k l}(f)$, and $\gamma_{k l}(f)+\gamma_{k^{\prime} l^{\prime}}(f)-i \gamma_{k l^{\prime}}(f)+i \gamma_{k^{\prime} l}(f)=2 \beta_{k l}(f)$. Hence we have

$$
\Phi_{\mathrm{gen}}(f)=\Phi(T f)+\Psi(\Gamma f)+\ell(f)
$$

so that, at least formally, the two-particle operators $\Psi(\cdot)$ we have been working with are sufficiently general to reproduce the transformations (5.1).

As in the proofs in Section 3.2, we see that if the transformation $\Phi(f) \mapsto$ $\Phi_{\text {gen }}(f)$ is to be canonical, then the operator $T$ must be symplectic. In particular, one has

$$
\begin{gathered}
{\left[\Phi_{\mathrm{gen}}(f), \Phi_{\mathrm{gen}}(g)\right]=i \sigma(T f, T g)+2 i \Phi(b(T f, \Gamma g)-b(T g, \Gamma f))} \\
+4 i \Psi\left(P_{+} B(\Gamma f, \Gamma g)\right)+2 i \sigma(\Gamma f, \Gamma g)
\end{gathered}
$$

Thus, as before, requiring $\left[\Phi\left(h_{1}\right),\left[\Phi\left(h_{2}\right),\left[\Phi_{\text {gen }}(f), \Phi_{\text {gen }}(g)\right]\right]\right]=\left[\Phi\left(h_{1}\right)\right.$, $\left.\left[\Phi\left(h_{2}\right), i \sigma(f, g) I\right]\right]=0$ for arbitrary $h_{1}, h_{2} \in H$, we find $P_{+} B(\Gamma f, \Gamma g)=0$, which implies $\sigma(\Gamma f, \Gamma g)=0$. Therefore, we conclude that $\sigma(T f, T g)=\sigma(f, g)$, i.e. $T$ is symplectic.

Now, since $T$ is symplectic and $\sigma(\cdot, \cdot)$ is nondegenerate, $T$ is injective, and, assuming that $T^{-1}$ is defined on all of $H$ (in infinitely many dimensions, this is not always assured), we can write $\Phi_{\text {gen }}\left(T^{-1} f\right)=\Phi(f)+\Psi\left(\Gamma T^{-1} f\right)+$ $\ell\left(T^{-1} f\right)$, and $\left[\Phi_{\text {gen }}\left(T^{-1} f\right), \Phi_{\text {gen }}\left(T^{-1} g\right)\right]=\left[\Phi(f)+\Psi\left(\Gamma T^{-1} f\right), \Phi(g)+\right.$ $\left.\Psi\left(\Gamma T^{-1} g\right)\right]$. Since $T^{-1}$ is also symplectic, it follows that

$$
\left[\Phi_{\mathrm{gen}}\left(T^{-1} f\right), \Phi_{\mathrm{gen}}\left(T^{-1} g\right)\right]=i \sigma(f, g)
$$

so that the transformation $\Phi(f) \mapsto \Phi(f)+\Psi\left(\Gamma T^{-1} f\right)$ must be canonical. Thus, the resulting conditions on $\Gamma T^{-1} \equiv \Lambda$ (i.e. $\Gamma=\Lambda T$ ) are $\Lambda \in \mathscr{L}_{C C R}$ (i.e., see Theorem 3.2.11 and Proposition 3.3.1). To summarize, we have seen (at least 
formally) that necessary and sufficient conditions for the general quadratic transformation (5.1) to be canonical are that the operator $T$ defined in (5.2) is symplectic and together with the operator $\Gamma$ defined in (5.3) must satisfy $\Gamma T^{-1}$ $\in \mathscr{L}_{C C R}$.

We compare this result with the special case $\operatorname{dim} \mathscr{H}=1$ handled previously in the literature [3], where a general quadratic canonical transformation was understood as

$$
\begin{aligned}
& q \mapsto Q=a_{1}(q+y p)^{2}+a q+b p+a_{4}, \\
& p \mapsto P=a_{1} x(q+y p)^{2}+c q+d p+b_{4}
\end{aligned}
$$

with $a_{1}, a_{4}, b_{4}, a, b, c, d, x, y \in \mathbb{R}$ satisfying the conditions

$$
a d-b c=1, \quad y=\frac{b x-d}{a x-c} .
$$

In fact, this is subsumed in our class of transformations; explicitly, with $H$ spanned by the symplectic orthonormal basis $e$ and $J e \equiv e^{\prime}$, then our $\Phi_{\text {gen }}(e)$ becomes their $Q$ and $\Phi_{\text {gen }}\left(e^{\prime}\right)$ becomes $P$, if we set $\ell(e)=a_{4}, \ell\left(e^{\prime}\right)=b_{4}, T e=a e+b e^{\prime}, T e^{\prime}=$ $c e+d e^{\prime}$, and $\Gamma=\Lambda T$, with $\Lambda=\sigma(l, \cdot) l \otimes l, l=\lambda e+\lambda^{\prime} e^{\prime}, \lambda=a_{1}^{1 / 3}(a x-c)^{1 / 3}$, and $\lambda^{\prime}=$ $a_{1}^{1 / 3} \frac{b x-d}{(a x-c)^{2 / 3}} . T$ is symplectic, since $\sigma\left(T e, T e^{\prime}\right)=a d-b c=1$, and also $\Lambda \in \mathscr{L}_{C C R}$, since after pulling out the factor $\|\ell\|^{3}$, it is in standard form.

\section{Quadratic Transformations and Automorphisms of the Weyl Algebra}

It is known [13] that the coherent transformation $\Phi(f) \mapsto \Phi(f)+\lambda(f) \mathbb{1}, f \in$ $D(\lambda)$, induces an automorphism on the $C^{*}$-algebra $\mathscr{A}(D(\lambda))$ via $W(f) \mapsto$ $e^{\imath \lambda(f)} W(f)$. The same is true on the algebra $\mathscr{A}(D(T))$ for the symplectic transformation $\Phi(f) \mapsto \Phi(T f), f \in D(T)$, via $W(f) \mapsto W(T f)$, whenever $\mathrm{R}(T)=D(T)$. But the canonical quadratic transformations do not induce such automorphisms, in other words, in general there exists no automorphism $\alpha$ on $\mathscr{A}(D(\Lambda))$, such that $\pi_{F}(\alpha(W(f)))=e^{i \Phi_{\Lambda}(f)}$ (recall that $\pi_{F}$ denotes the Fock representation, so that $\left.\pi_{F}(W(f))=e^{i \Phi(f)}\right)$, for all $f \in D(\Lambda)$. This is due to the fact that in general the operator $e^{i \Phi_{\Lambda}(f)}$ simply does not lie in the norm closure of the set $\left\{e^{i \Phi(f)} \mid f \in\right.$ $H\}$. This assertion is demonstrated in [18] for the simplest nontrivial example $-H$ two-dimensional with symplectic orthonormal basis $\{e, J e\}$ and $\Lambda f=\lambda \sigma(e$, f) $e \otimes e, \lambda>0$. 


\section{Transformations of Order Higher Than Quadratic}

It is, of course, natural to consider higher-order transformations which go beyond the quadratic [22]. Define an $r$-particle operator $\Psi^{(r)}$ by

$$
\Psi^{(r)}(F) \varphi \equiv \sum_{i=1}^{n} \alpha_{i}: \Phi\left(f_{1 i}\right) \Phi\left(f_{2 i}\right) \cdots \Phi\left(f_{r i}\right): \varphi, \quad \varphi \in F_{0}
$$

for $F=\sum_{i=1}^{n} \alpha_{i}\left(f_{1 \imath} \otimes f_{2 i} \otimes \cdots \otimes f_{r i}\right)\left(r, n \in \mathbb{N}, \alpha_{i} \in \mathbf{R}, f_{k i} \in H\right)$. As with the two-particle operator $\Psi$, one finds that the $\operatorname{map} F \mapsto \Psi^{(r)}(F) \varphi$ is bounded when $\varphi \in \mathscr{F}_{0}$, so that again $\Psi^{(r)}(F) \varphi$ can be continuously extended to all of $H^{r}$. Because of the normal ordering in $(5.5), \Psi^{(r)}(F)=\Psi^{(r)}\left(P_{+} F\right)$ once again, and $\Psi^{(r)}(F)$ is a symmetric operator. Thus a canonical transformation of order $r$ could be defined mathematically as $\Phi(f) \mapsto \overline{\Phi(f)+\Psi^{(r)}(\Lambda f)}, f \in D(\Lambda)$, where $\Lambda$ is a densely defined linear operator from $H$ to $P_{+} H^{r}$ such that the transformation is canonical.

However, the techniques of this paper break down at two important points for $r>2$. It turns out that the norm of the map $F \mapsto \Psi^{(r)}(F) \varphi$ with $\varphi \in \mathscr{F}_{0}$ involves a factor $\left(n_{\varphi}+r\right)^{r / 2}$, which for $r>2$ prevents the finite-particle vectors from being analytic vectors of $\Psi^{(r)}(F)$. It is therefore not evident that $\Phi(f)+\Psi^{(r)}(F)$ is self-adjoint, nor that the exponentiation of such a transformation would result in a representation of the corresponding Weyl algebra. In fact, showing that an operator like $\Psi^{(r)}(F)$ is essentially self-adjoint for $r>$ 2 is an old and difficult problem in quantum field theory. One possibility to deal with these difficult operator-theoretic questions is to go to the $Q$-space formalism, as in Section 4.3. But in order to do that usefully, it would be necessary to use the additional information $\Lambda \in \mathscr{L}_{C C R}^{(r)}$ to bring the operator $\Lambda$ into a suitable standard form, which leads to the second sticking point.

Here the problem is that if $\Lambda$ is to be brought into standard form (we write only the case $r=3$ )

$$
\Lambda f=\sum_{i, j, k} \sum_{l} \lambda_{i j k l} \sigma\left(n_{l}, f\right) n_{i} \otimes n_{j} \otimes n_{k}
$$

with a symplectic orthonormal basis $\left\{n_{K}\right\}$ in $H$, then because of $\mathrm{R}(\Lambda) \subseteq N \otimes N \otimes$ $N, \sigma(m, n)=0 \forall m, n \in \mathbf{N}$ it would be necessary for the entire vector

$$
\sum_{p, q, s, u, v, w} \alpha_{p q s} \beta_{u v w} \sigma_{s u} e_{p} \otimes e_{q} \otimes e_{v} \otimes e_{w}
$$

to vanish, and not just its symmetric component. We did not succeed in deriving 
this condition from the requirement that the transformation be canonical (see [18] for further details).

It should also be pointed out that the commutator of an $r$ - and an $s$-particle operator yields a sum consisting of an $(r+s-2)-$, an $(r+s-4)-$, an $(r+s-6)-$ particle operator, and so forth. Thus the operators $\mathbb{1}, \Phi(f)(f \in H)$, and $\Psi^{(2)}(F)\left(F \in P_{+} H^{2}\right)$ form a Lie algebra, whereas a Lie algebra which contains a cubic (or higher) operator also contains all higher-order operators. Thus, both algebraically and analytically, the quadratic case has a special status.

\section{Some Applications of Canonical Quadratic Transformations}

As previously indicated in the introduction, an important application of quadratic transformations will be to generate non-Fock representations in which one can more suitably model and compute quantities of physical interest. This is exemplified heuristically in [17] and especially in [16]. Such transformations should also be of use in constructing exactly soluble models and have applications in the field of special functions (see [5] and the references given there).

Next consider the following formal Hamilton operator:

$$
\begin{aligned}
H & =\sum_{k=1}^{\infty}\left\{\frac{\omega_{k}}{2}\left(p_{k}^{2}+q_{k}^{2}-1\right)+\sqrt{2} \mu_{k}\left(p_{k}: q_{k}^{2}:+: q_{k}^{2}: p_{k}\right)+4 \nu_{k}\left(: q_{k}^{2}:\right)^{2}\right\} \\
& =\sum_{k=1}^{\infty}\left\{\omega_{k} a_{k}^{*} a_{k}+i \mu_{k}\left(a_{k}^{* 3}+a_{k}^{* 2} a_{k}-a_{k}^{*} a_{k}^{2}-a_{k}^{3}\right)+\nu_{k}\left(a_{k}^{* 2}+2 a_{k}^{*} a_{k}+a_{k}^{2}\right)^{2}\right\}
\end{aligned}
$$

where $\omega_{k}, \mu_{k}, \nu_{k} \in \mathbb{R}$ and $\omega_{k}, \nu_{k}>0$. The canonical quadratic transformation

$$
q_{k} \mapsto q_{k}{ }^{\prime}=q_{k}, \quad p_{k} \mapsto p_{k}{ }^{\prime}=p_{k}+\lambda_{k}: q_{k}^{2}:
$$

yields

$$
\begin{aligned}
H \mapsto H^{\prime}= & \sum_{k=1}^{\infty}\left\{\omega_{k} a_{k}^{*} a_{k}+i\left(\mu_{k}+\frac{\omega_{k} \lambda_{k}}{2 \sqrt{2}}\right)\left(a_{k}^{* 3}+a_{k}^{* 2} a_{k}-a_{k}^{*} a_{k}^{2}-a_{k}^{3}\right)\right. \\
& \left.+\left(\nu_{k}+\frac{\mu_{k} \lambda_{k}}{\sqrt{2}}+\frac{\omega_{k}}{8} \lambda_{k}^{2}\right)\left(a_{k}^{* 2}+2 a_{k}^{*} a_{k}+a_{k}^{2}\right)^{2}\right\},
\end{aligned}
$$

which with respect to $H$ merely has a different coupling constant. But then with the choice $\lambda_{k}=-2 \sqrt{2} \frac{\mu_{k}}{\omega_{k}}$, the transformed Hamiltonian becomes purely quartic:

$$
H^{\prime}=\sum_{k=1}^{\infty}\left\{\omega_{k} a_{k}^{*} a_{k}+\left(\nu_{k}-\frac{\mu_{k}^{2}}{\omega_{k}}\right)\left(a_{k}^{* 2}+2 a_{k}^{*} a_{k}+a_{k}^{2}\right)^{2}\right\} .
$$


This operator $H^{\prime}$, for suitable choice of the energies and coupling constants $\omega_{k}$, $\mu_{k}, \nu_{k}$ is well-defined in Fock space and bounded from below, whereas the original Hamiltonian may not be. And if, in fact, the transformation (5.6) is not unitarily implementable, i.e. when $\sum_{k=1}^{\infty} \lambda_{k}^{2}$ diverges, but $H^{\prime}$ is well-defined on Fock space, then $H$ is not a well-defined operator on Fock space. If, on the other hand, the quadratic transformation is unitarily implementable, then the original operator $H$ is well-defined on Fock space and bounded from below (and the physics of the system described by $H$ may just as well be described by $H^{\prime}$ ) . Thus, such quadratic transformations may be used to transfer information from one representation to another.

Finally, we mention that the $Q$-space formulation of quantum field theory implies that every result on the unitary equivalence (resp. nonequivalence) of a given representation of the CCR with the Fock representation yields a corresponding result of the equivalence (resp. nonequivalence) of measures on infinite-dimensional spaces. However, we shall not take more space to spell out these results here. For further applications, see Chapter 9 of [18].

\section{References}

[1] Berezin, F. A., The Method of Second Quantization. Academic Press, New York, 1966.

[2] Bratteli, O. and Robinson, D. W., Operator Algebras and Quantum Statistical Mechanics, II, Springer-Verlag, New York, Heidelberg and Berlin, 1981.

[3] Colegrave, R. K. and Bala, P., Nonlinear canonical transformations, J. Phys. A, 18 (1985). 779-791.

[4] Cook, J. M., The mathematics of second quantization. Trans. Amer. Math. Soc., 74 (1953). 222-245.

[5] Deenen. J., Generators for nonlinear canonical transformations, J. Phys. A, 24 (1991). 3851-3858.

[6] Dirac, P. A. M., Emission and absorption of radiation, Proc. Roy. Soc. London, 114 (1972), 248.

[7] Emch. G. G.. Algebraic Methods in Statistical Mechanics and Quantum Field Theory. John Wiley \& Sons, New York, 1972.

[8] Gårding L. and Wightman, A. S., Representations of the commutation relations, Proc. Nat. Acad. Sci., 40 (1954), 622-626.

[9] Haag, R., Local Quantum Physics, Springer-Verlag, New York, Heidelberg and Berlin, 1992.

[10] Hegerfeldt, G. C., Gårding domains and analytic vectors for quantum fields, J. Math. Phys., 13 (1972), 821-827.

[11] Kastler, D., The $C^{*}$-algebras of a free boson field, Comm. Math. Phys., 1 (1965), 14-48.

[12] Klauder J. R. and Skagerstam, B.-S., Coherent States: Applications in Physics and Mathematical Physics, World Scientific, Singapore, 1985.

[13] Manuceau, J., $C^{*}$-algèbre de relations de commutation, Ann. Inst. Henri Poincaré, 8 (1968), 139-161.

[14] Manuceau J. and Verbeure, A., Quasi-free states of the CCR-algebra and Bogoliubov transformations, Comm. Math. Phys., 9 (1968), 293-302.

[15] Manuceau, J., Sirugue, M., Testard, D. and Verbeure, A., The smallest $C^{*}$-algebra for canonical commutation relations, Comm. Math. Phys., 32(1973), 231-243. 
[16] Moshinsky, M., Seligman, T. H. and Wolf, K. B., Canonical transformations and the radial oscillator and Coulomb problems, J. Math. Phys., 13(1972). 901-907.

[17] Polley L. and Ritschel, U., Second-order phase transition in $\lambda \phi_{2}^{4}$ with non-Gaussian variational approximation, Phys. Lett. B, 221 (1989), 44-48

[18] Proksch, M., Quadratische Kanonische Transformationen von Quantenfeldern, Ph. D. thesis, University of Würzburg, 1992.

[19] Reed, M., A Gårding domain for quantum fields, Comm. Math. Phys., 14 (1969), 336-346.

[20] Reed, M. and Simon, B., Methods of Modern Mathematical Physics, I. Functional Analysis, Academic Press, New York, 1972.

[21] Methods of Moderm Mathematical Physics, II . Fourier Analysis, Self-Adjointness, Academic Press, New York, 1975.

[22] Reents, G. and Summers, S. J., Beyond coherent states: higher order representations, in: On Klauder's Path: A Field Trip, edited by Emch, G. G., Hegerfeldt, G. C. and Streit, L., World Scientific, Singapore, (1994), 179-188.

[23] - Manuscript in preparation.

[24] Segal, I. E., Tensor algebras over Hilbert spaces, I, Trans. Amer. Math. Soc., 81 (1956). 106-134.

[25] Sewell, G. L., Quantum Theory of Collective Phenomena, Oxford University Press, Oxford, 1986.

[26] Shale, D., Linear symmetries of the free boson field. Trans. Amer. Math. Soc., 103 (1962), 149-167.

[27] Simon. B., The $P(\Phi)_{2}$ Euclidean (Quantum) Field Theory, Princeton University Press, Princeton, 1974.

[28] Slawny, J., On factor representations and the $C^{*}$-algebra of canonical commutation relations, Comm. Math. Phys., 24 (1971), 151-170. 